\title{
REFERENCE
}

\section{Second Workshop on Wireless Sensing: Proceedings}

James Gilsinn

Kang Lee

Rick Schneeman

Hui-Min Huang

U.S. DEPARTMENT OF COMMERCE

Technology Administration

National Institute of Standards and Technology

100 Bureau Drive

Gaithersburg, MD 20899

QC

100

. $\mathbf{U 5 6}$

\#6930 



\title{
Second Workshop on Wireless Sensing: Proceedings
}

\author{
James Gilsinn \\ Kang Lee \\ Rick Schneeman \\ Hui-Min Huang
}

U.S. DEPARTMENT OF COMMERCE

Technology Administration

National Institute of Standards and Technology 100 Bureau Drive

Gaithersburg, MD 20899

November 2002

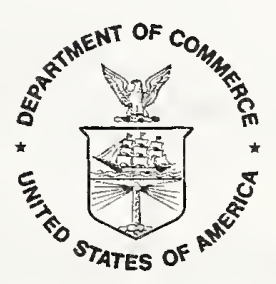

U.S. DEPARTMENT OF COMMERCE Donald Evans, Secretary

TECHNOLOGY ADMINISTRATION

Phillip J. Bond, Under Secretary for Technology

NATIONAL INSTITUTE OF STANDARDS AND TECHNOLOGY

Arden L. Bement, Director 



\section{Second Workshop on Wireless Sensing Proceedings}

Sensors Expo \& Conference October 4, 2001

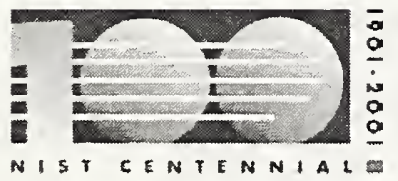
Philadelphia, PA

NIST CENTENNIALE

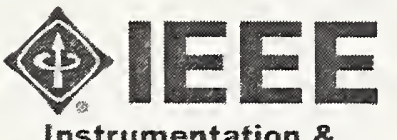
Instrumentation \& Measurement Society

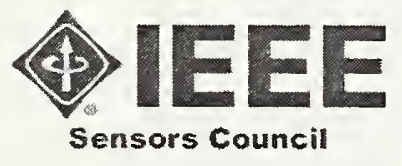

James Gilsinn, Intelligent Systems Division

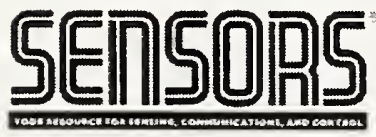

Kang Lee,

Manufacturing Metrology Division

Rick Schneeman, Manufacturing Metrology Division

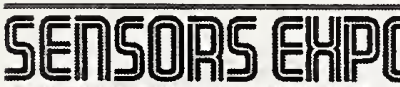
Hui-Min Huang, B CONFElizTTE Intelligent Systems Division 



\section{Table of Contents}

1 Overview of the Second Wireless Sensing Workshop ............................... 1

$2 \quad$ Issues \& Discussions ........................................................................... 3

2.1 Workshop on IEEE 1451.2 Specification Enhancement .............................................

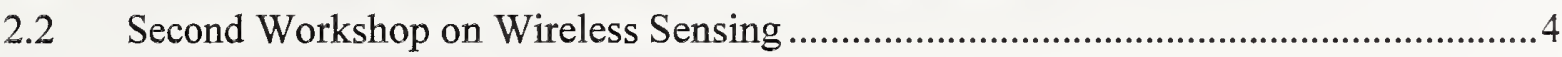

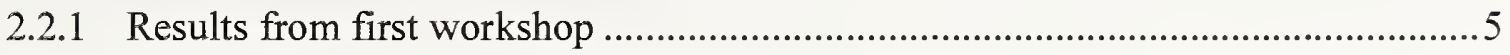

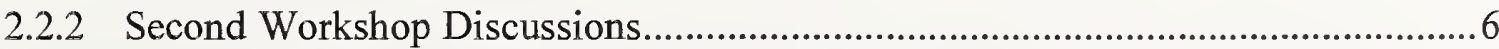

3 Presentations from "Enhancement of IEEE 1451.2, A Smart

Transducer Interface Standard" "................................................................. 8

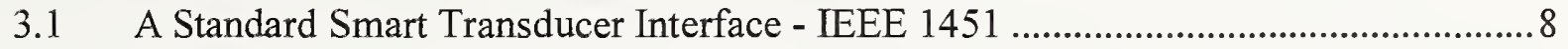

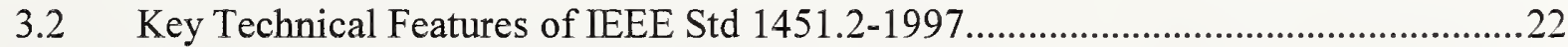

3.3 Proposed Enhancements to the IEEE 1451.2 Standard for Smart Transducers ...........31

3.4 Requested Changes to the IEEE 1451.2 Standard for Smart Transducers ...................49

3.5 A Sensor Manufacturer's Perspective ..........................................................................53

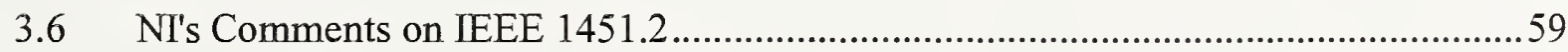

3.7 Proposed Enhancements to the IEEE 1451.2 Standard for Smart Transducers ..........65

4 Presentations From "Second Workshop on Wireless Sensing"............. 76

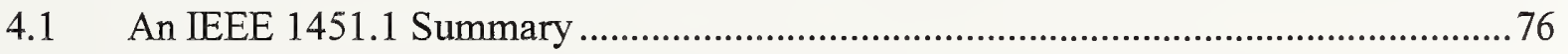

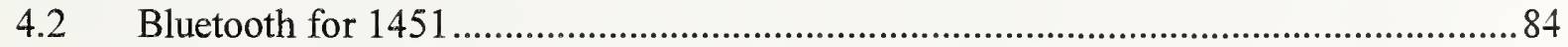

4.3 Synopsis on Wireless Ethernet (IEEE 802.11) ...................................................... 97

4.4 Reliable Wireless Connectivity for Sensing \& Control Applications .........................103

4.5 IEEE 802.15.4 Low Rate Personal Area Networks................................................. 122

4.6 Wireless Implementations for an Open Standard Sensor Bus ....................................128

4.7 Review Issues \& Discussions from First Workshop ...............................................135

$5 \quad$ Workshop Agenda .................................................................................... 143

$6 \quad$ List of Attendees.......................................................................................... 144 


\section{DISCLAIMER:}

Commercial equipment and software referred to in this document are identified for informational purposes only, and does not imply recommendation of or endorsement by the National Institute of Standards and Technology, nor does it imply that the products so identified are the best available for the purpose. 


\section{Overview of the Second Wireless Sensing Workshop}

The second Workshop on Wireless Sensing was held on October 4, 2001, at the Sensors Expo \& Conference at the Philadelphia Convention Center in Philadelphia, PA. The National Institute of Standards and Technology (NIST), SENSORS magazine, Sensors Conference, Institute of Electrical and Electronics Engineers (IEEE) Instrumentation and Measurement Societyís Technical Committee on Sensor Technology (TC-9), and IEEE Sensors Council cosponsored the workshop. NIST is an agency of the U.S. Department of Commerceís Technology Administration. Its mission is to help increase U.S. industry competitiveness through advanced research, standards, and technology collaboration.

Recently, there has been considerable interest from industry and government in applying wireless technology to sensor-based applications. Wireless technology has seen an explosion over the last couple years with the introduction of Bluetooth and higher speed wireless Ethernet. These technologies are moving from the office environment to the industrial plant floor as engineers discover how flexible and easy to use they are.

The Sensor Development and Application Group at NIST has been working with industry and IEEE to establish IEEE 1451, titled i A Standard for a Smart Transducer Interface for Sensors and Actuatorsî. In response to the industryís interest in wireless sensing, NIST initiated, cosponsored and conducted a workshop in June of 2001. The workshop in June provided NIST with valuable information as to the direction it should take in helping develop a wireless version of IEEE 1451. At the first workshop, it was proposed that a second workshop be held in order to discuss some of the more important issues as well as work on forming a study group for wireless 1451. The second workshop provided a more concentrated format in order to discuss the issues.

The format for the workshop consisted of a meeting of the IEEE 1451.2 committee for the first couple hours and the rest of the day taken up by the wireless sensing workshop. Eighteen people attended the IEEE 1451.2 committee meeting split approximately 4 to 3 to 3 between vendors, users, and government employees respectively. Twenty-three people attended the wireless sensing workshop split approximately 4 to 4 to 2 between vendors, users, and government employees respectively. Overall attendance at the conference was down by over $70 \%$ compared with the June 2001 conference. It is assumed that the severe drop in attendance of the second workshop compared to the first is due to the events of September 11th and not due to the lack of interest in the subject matter.

The main purpose of the 1451.2 committee meeting was to review and update any parts of the four-year old standard that the members felt needed updating. The meeting consisted of a series of papers with free discussions during and after the papers. Most of the discussion centered on the Transducer Electronic Data Sheet (TEDS) and how it has become one of the most important components of IEEE 1451. Another main topic discussed was the separation of hardware from software in the standard. Other topics discussed were the industries view of IEEE 1451 and the real-time aspects of the standard.

The major portion of the day was taken up by the second Workshop on Wireless Sensing. The workshop was more structured that the 1451.2 meeting earlier in the day, consisting of a series of 
presentations and then discussions afterward. Presentations were made on some implementations of IEEE 1451 or wireless technology, Bluetooth, wireless Ethernet (IEEE 802.11), and a standard under development in the IEEE 802.15.4 committee. A summary of the previous workshop and its results was then presented. After which, discussions started as to the direction the group should take in developing a wireless version of IEEE 1451.

As a direct result of this workshop, a working group was formed to begin developing a proposed IEEE 1451.5 wireless standard. As a starting point for the possible standard, one company that attended the workshop offered to provide one of its patents to the group. The working group was then tasked with coming up with a mission and purpose in order to apply for a formal study group through IEEE. 


\section{Issues \& Discussions}

\subsection{Workshop on IEEE 1451.2 Specification Enhancement}

A workshop focused on the enhancement of the specification of the IEEE 1451.2 standard took place before the second workshop on wireless sensing was conducted. Although this group is separate from the wireless sensing group, many of the 1451.2 attendees are concerned about a wireless extension to the 1451 standard and attended the wireless sensing workshop. Eighteen people attended the working group meeting with the distribution being split $44 \%$ vendors, $28 \%$ users, and $28 \%$ government.

With the initial IEEE 1451.2 standard being published four years earlier, the main purpose of this meeting was to review the standard as it is right now and determine if changes should be made and what should be considered. The 1451.2 workshop consisted of a series of presentations and open discussions during and after the presentations.

The Transducer Electronic Data Sheet (TEDS) has emerged as the most important part of the of the IEEE 1451.2 standard thus far. Many companies are trying to implement digital interfaces to their products, and others are trying to incorporate a TEDS-like system, but these are not standardized. Only the TEDS provides a common way for sensors to store calibration and identification information and communicate that over a sensor network. One of the major drawbacks with the TEDS currently is the disharmony between the TEDS of the different dot standards (refers to the IEEE 1451.2, P1451.3, and P1451.4 standards either in place or being developed). The different dot committees and standards have much different requirements and the version of TEDS that each of them uses is somewhat different at this point.

Some users look at IEEE 1451 as an unnecessary layer of abstraction being applied to their sensors and/or sensor networks. The attendees felt that these users need to be shown that IEEE 1451 has benefits that the users might easily overlook. The plug-and-play aspects of the standard and the TEDS need to be promoted more in order to show how they will reduce the upgrade and maintenance costs for the overall sensor system as well as reducing the time for individual sensor installation and configuration. While no formal survey has been conducted, conversations with system integrators and users have indicated that setup and configuration of complex systems can be a major undertaking. With many industrial systems containing hundreds or thousands of sensors, it can take a large amount of time with many mistakes in order to configure each individual sensor, the appropriate drivers, and the sensor parameters into the control system. With IEEE 1451 and specifically TEDS, these times can be greatly reduced and more accurate.

One of the main topics discussed was redefining the 1451.2 standard such that the hardware and software portions were split. The dot 2 , dot3, and dot 4 committees (referring to the 1451.2, 1451.3, and 1451.4 committees or standards) have all been developed in relative seclusion from one another. Even though the groups do have some common members, each standard has a different goal. Therefore, three very different standards addressing different needs to interface sensor, network, and instrument have emerged. IEEE 1451.2 provides interface specification for connecting sensors and microprocessors or networks. P1451.3 specifies interfaces for highspeed distributed systems for reading a large array of sensors. While, P1451.4 defines a set of 
specifications for connecting sensors and instruments via a very simple 2 to 4 wire interface. A potential confusion could occur because companies might have difficulty in choosing what standard to develop their products. Some efforts are needed to educate the sensor community.

By redefining the standard, the software portions of the dot2, dot3, and dot 4 standards would be separated from the hardware portions. The software components of these standards could be made more consistent with less difficulty, especially the model of the transducer and the communications protocol. The hardware portions of the standard would then be separated by the different dot standards. The different dot standards could also include modifications to the software portion as necessary, but would not be required to do so.

The dot 3 committee has begun the process of extracting the hardware potion of its standard from the software portion. They are following a modified version of the International Standards Organization (ISO) 7-layer model with fewer layers. This allows the committee to define the standard in a way that allows for it to be split up at some future date with less difficulty than if they hardware and software were integrated. The dot 2 committee also feels that this is a good idea, and is going to try and move in that direction.

An additional topic that was brought up in the discussions was the fact that some people might have difficulty understanding the overall purpose of IEEE 1451. Many developers and users feel that IEEE 1451 is just another communications standard, and though it is an interesting idea, will not work for them since there are many other industrial and sensor networks that they may already use. The committee agreed that IEEE 1451 should not be the platform to create a new standard for communications. It should standardize the data model, control model, and electronic data sheets, then provide the tools to map these models over any network or communication protocol. The IEEE 1451 committee and the vendors developing products for 1451 need to make it more clear that the standard is not another communication network, but is a way for sensors to connect to any communication network in a standard way. The standard allows any 1451 compliant device to attach to any network and report its data using a standard method. Any computer or device capable of reading the 1451 "language" can read that data and make use of it.

One last topic mentioned was the term "real-time" needs to be used carefully. To some industries and users, real-time may mean doing full motion audio/video over a network or it may mean reading 1 byte of data at $100 \mathrm{~Hz}$. Most things in the sensor world can be done with a bandwidth of 19.2 kilobits per second (kbps). RS-232 can handle these speeds very well, and still remains a very popular choice for a sensor interface.

\subsection{Second Workshop on Wireless Sensing}

This workshop was held on October 4, 2001 at the 2001 Fall Sensors Expo \& Conference at the Philadelphia Convention Center in Philadelphia, PA. It was a follow on to the first workshop held in Chicago, IL on June 4, 2001. Twenty-three people attended this workshop with a distribution of $39 \%$ vendors, $39 \%$ users, and $22 \%$ government. The second workshop was much smaller than the first workshop, probably due to the tragic events of September 11, 2001 and not to the lack of interest by people in the sensor community. 
The workshop consisted of a series of presentations either summarizing material from the first workshop or presenting new material since the first workshop. These presentations were followed by a short discussion of the previous workshop's results and a long discussion on how to go about developing a wireless sensing standard.

\subsubsection{Results from first workshop}

As part of the previous workshop discussions, the issue of where the wireless communications should be located was brought up. The first workshop provided two places for the wireless communications to be used in the overall sensor system: 1) at the Sensor Transducer Interface Module (STIM) level, and 2) at the Network Capable Application Processor (NCAP) node level. In addition to providing those two examples, the first workshop decided that the second of these was the better of the two. The attendees of the second workshop felt that both cases were valid, and the choice of one over another was more application dependant than one being better in all situations. The two different cases would provide many different requirements for the sensors, including more or less intelligence, power, or cost.

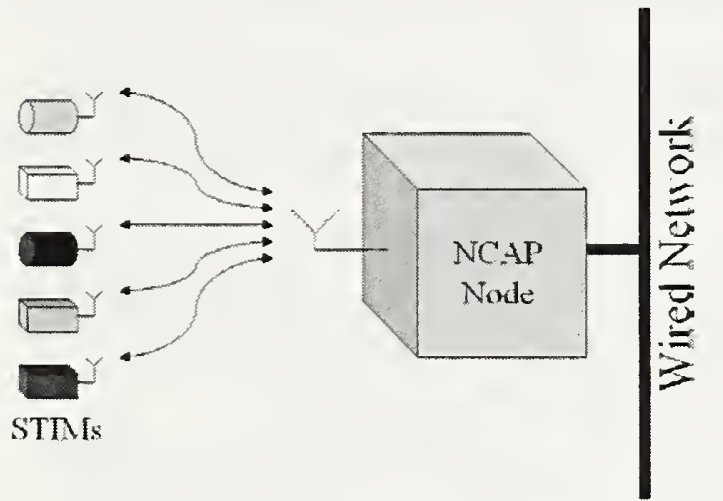

Figure 1 - Wireless STIMs

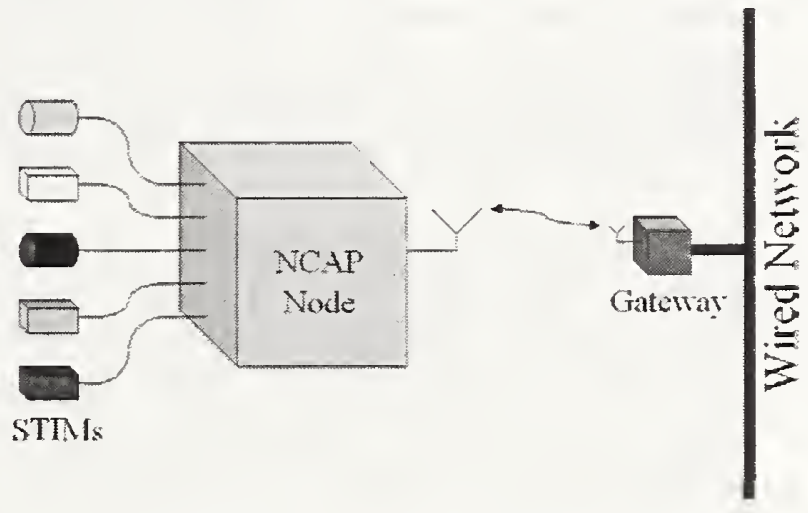

Figure 2 - Wireless NCAP Node

Battery lifetime was also discussed in the results from the first workshop. Some people commented that while $100 \mathrm{~mW}$ may wear down a battery during continuous use, reducing the frequency and length of transmission were ways to use $100 \mathrm{~mW}$ of power and extend battery lifetime. Using $100 \mathrm{~mW}$ of transmission power is useful in many cases where signal noise is encountered. Lower transmission power signals can be swamped by even small amounts of noise in the area. By using $100 \mathrm{~mW}$, the signal can be blasted through some noise and received at greater distances.

The attendees felt that the wireless standard should be defined such that the hardware component of the standard should be removed from the software component. This view is similar to the discussion held at the IEEE 1451.2 meeting earlier in the day, in which the standards would be separated on their hardware portions, and would not include a software component unless necessary. Additional examples were given with respect to the IEEE 802 committee and specifically the 802.11 (wireless Ethernet) and 802.15 (Bluetooth-related) subcommittees. 


\subsubsection{Second Workshop Discussions}

Based the first and second workshop, it appears that a wireless sensor standard is something that the sensor industry would like to see. It would provide wireless sensor interoperability, easier wireless sensor upgrades, plug-and-play capability, scalability, and lower overall cost for the wireless sensor network. These benefits would not only be for the end user of the product, but also to system integrators who would not have to work with a multitude of de-facto or somewhat related standards to develop their products. IEEE 1451 will serve as the basis for this new standard, but there will be enough differences between the current versions of the standard and the possible requirements of a wireless version that it will justify adding a new dot standard to the 1451 family. The new standard will incorporate existing wireless communication standards in order to speed up the development time, but this will also add additional complexity to the overall design. Different technologies will be reviewed for their appropriateness and capabilities for this particular application.

While TEDS-like enabled products have been available from sensor manufacturers for some time, IEEE 1451 TEDS is still in its infancy. Some IEEE 1451.2-based products have been developed with this capability, but they havenit been widely implemented in the market. The group felt that it would be a good idea for the IEEE 1451.2 committee to look into separating out the TEDS portion of the standard in order to gain wider acceptance. This would also allow for better development of the wireless standard since the TEDS would not have to be redefined for the wireless standard, just modified with a part dedicated to the wireless communications.

One of the participants in these discussions, a company named Axxon, is a vendor with a long history of developing wireless sensors. Axxon believed that developing this standard would not reduce their marketability, as some vendors may believe, but would actually increase their profitability. Axxon would be able to spend less time and effort developing new communications protocols and electronics for new applications, speeding up their overall timeto-market for new products. Axxon is willing to release one of their patents to the committee in order to develop a wireless sensor standard quicker than developing one from scratch. They would have to discuss the issue with their legal department, however, there did not seem to be any issues as the discussions were taking place.

After Axxon offered to release one of their patents, this sparked a discussion of patent law and the legal ramifications of such an action. Some of the participants brought up the point that Axxon would not have to freely distribute the patent without expecting any compensation. They could release the patent with the expectation of royalties or some sort of licensing fee. Axxon will have to make a decision on this before releasing the patent to the committee. Care must be taken to avoid any legal problem when developing a standard based on any patent not to exclude or require any particular companies from the standard.

After it appeared that the substantive discussions were winding down, the issue of forming the working group under the IEEE 1451 committee was undertaken. Although all the people that attended the meeting were interested in the wireless smart sensors standard, some of the participants were already involved in other committees and could not spare the time to be part of this group. A meeting of the IEEE PAR (Project Authorization Request) review board will be held on December 15th, and this group will need to put together its plan forty days prior to this 
meeting. Until the time when the PAR committee officially approves the formation of a working group on this subject, Kang Lee of NIST, the Chair of the Sensor Technology Technical Committee, will initiate a study group. Steven Chen of $3 \mathrm{e}$ Technology Inc. was proposed to be the head of this study group.

While it may be good for the committee to look at a wide range of technologies and applications in its research, the scope of the working group should be limited in order to make it feasible to develop a standard in a reasonable amount of time. By including too many members from too wide a range of applications, the group may run into to trouble of the extremely different requirements of the various applications. Industries like security tags and RFIDs would seem to be applicable to a wireless sensor standard; they have many different requirements that require them to be excluded from the planned committee distribution. It will be important for the group to maintain ties with both the IEEE 802.11 and 802.15 committees, though. These two standards have been discussed as possible physical layers for the wireless smart sensor standard, and this study group will need to keep abreast of the status of those standards committees. Data synchronization was an issue that was briefly discussed. Kang Lee pointed out that the IEEE P1588 working group is developing a proposed i Standard for Precise Clock Synchronization in Networked Measurement and Control Systemsî to address this issue.

The study group will need to provide a timeframe for developing this standard to the PAR review board as part of its application. A year may be too short to develop the wireless standard, but the time should be limited in order to promote current technology in the standard. All committee efforts take longer than initially expected, so that should also be taken into account when writing up the application. Axxon was asked to provide a strawman for a standard to the group members in order to begin the process of developing the standard. This allows the group to work from a starting point, and can usually speed up the process considerably. Other sensor vendors and chip manufacturers will need to be included in this group as well. Without the development resources of sensor vendors and chipmakers, it will be unlikely that the standard will gain wide acceptance. It would take vendors a long period of time to ramp up development for the particular standard if they adopted it at all.

The workshop broke up well after the scheduled time with the participants feeling that the standard could and should be developed. The group planned to work quickly over the next couple weeks in order to file the application for the IEEE working group. There was no planned follow-up workshop now that the group planned on actually developing the standard through the IEEE process. 


\title{
3 Presentations from "Enhancement of IEEE 1451.2, A Smart Transducer Interface Standard"
}

\subsection{A Standard Smart Transducer Interface - IEEE 1451}

\section{A Standard Smart Transducer Interface \\ - IEEE 1451}

\author{
Robert Johnson, Telemonitor, Inc. \\ Kang Lee, NIST \\ James Wiczer, Sensor Synergy, Inc. \\ Stan Woods, Agilent Technologies, Inc.
}

Agenda

- Measurement and control systems

- Smart transducers

- Intioduction to IEEE 1451

- Benefits of the 1451 standard

- Contacts for further information 


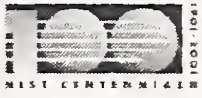

\section{Measurement/control system}
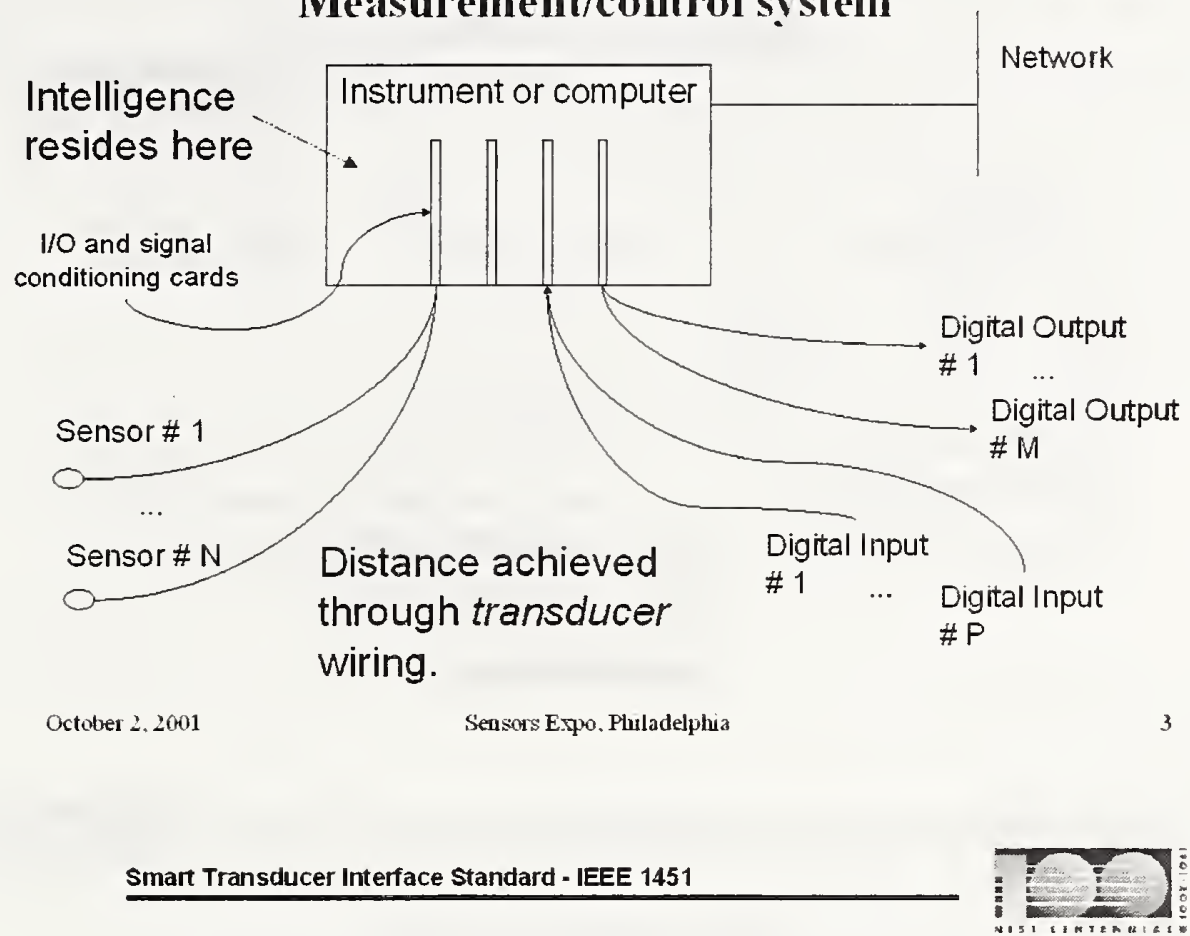

\section{Distributed measurement/control system}

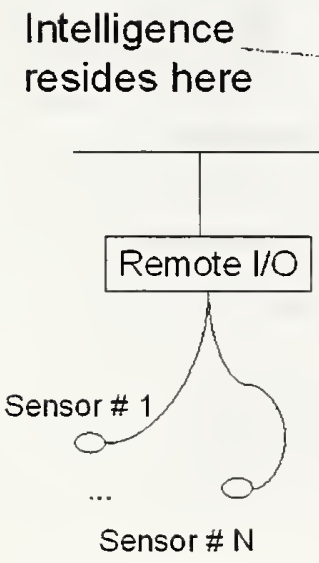

October 2. 2001

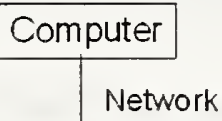

Distance achieved through network wiring.
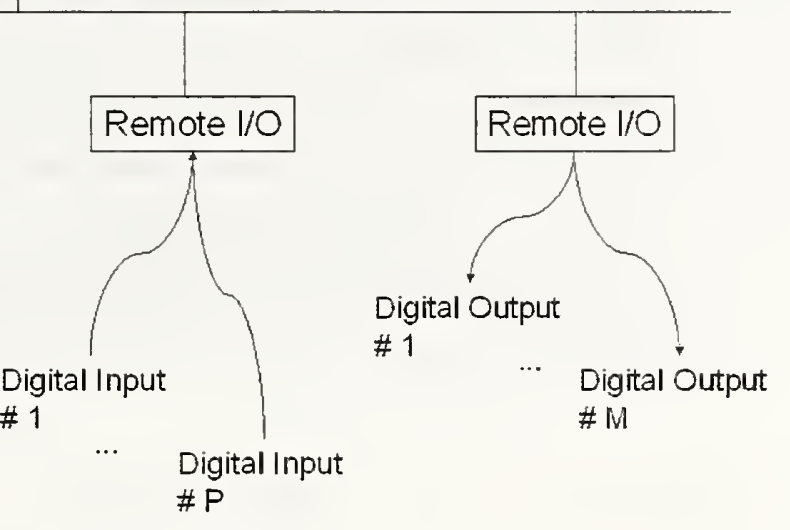

Seusors Expo. Philadelulua 
Distributed smart sensor/actuator system

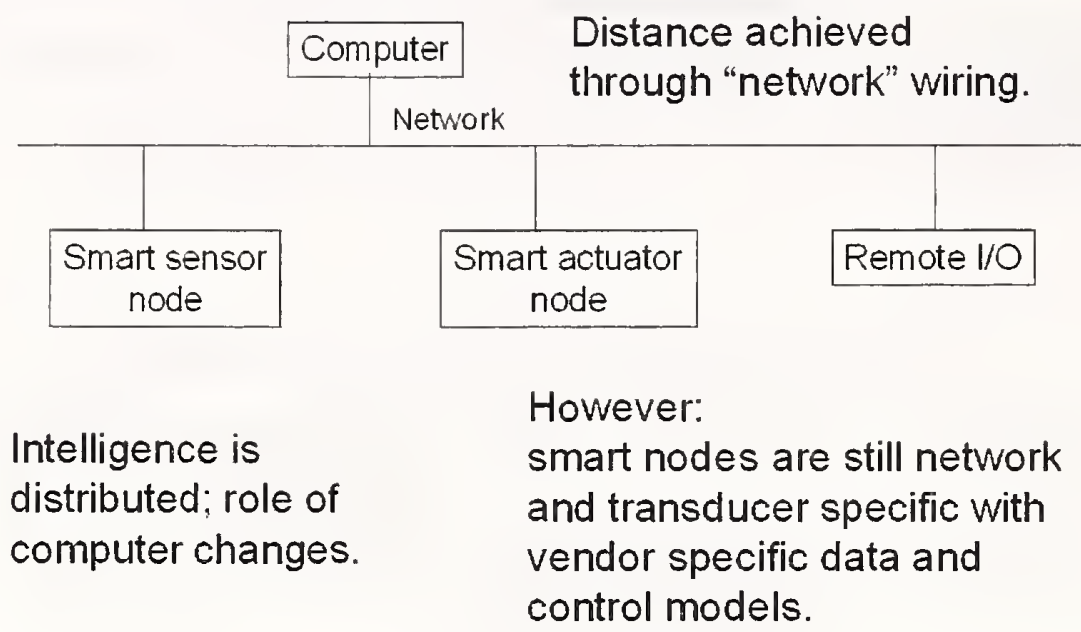

\section{Main goals for 1451}

- Derelop netrork independent and rendor independent trans ducer interfaces.

- Allow transducers to be replaced mored with minimum effort.

- Eliminate error prone. manual system configuration steps.

- Support a general transducer data. control timing. configuration and calibration model.

- Derelop Transducer Electronic Data Sheets that remain together with the transducer during normal operation. 


\section{Network independent transducers}

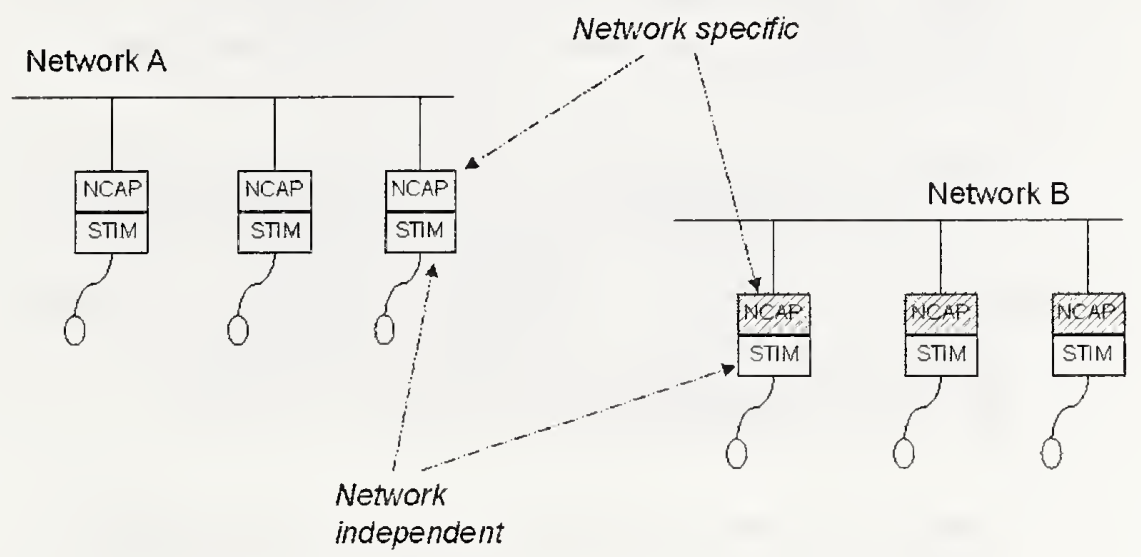

Desirable functions in sensors that provide "smarts"

- Self-identification, self-diagnostic.

- Output digital data in standard engineering units.

- "Time aware" for timestamping and correlation

- Software functions, e.g.:

- signal processing and data logging

- measurements derived from multi-channels

- Conforming to a standard data and control protocol 


\section{What standards are being developed?}

- IEEE Std 1451.1-1999, Network Capable Application Processor (NCAP) Information Model for smart transducers -- Published standard.

- IEEE Std 1451.2-1997, Transducer to Microprocessor Communication Protocols and Transducer Electronic Data Sheet (TEDS) Formats -- Published standard.

- IEEE P1451.3, Digital Communication and Transducer Electronic Data Sheet (TEDS) Formats for Distributed Multidrop Systems -- Being developed

- IEEE P1451.4, Mixed-mode Communication Protocols and Transducer Electronic Data Sheet (TEDS) Formats --Being developed

\section{A general model of a smart sensor}

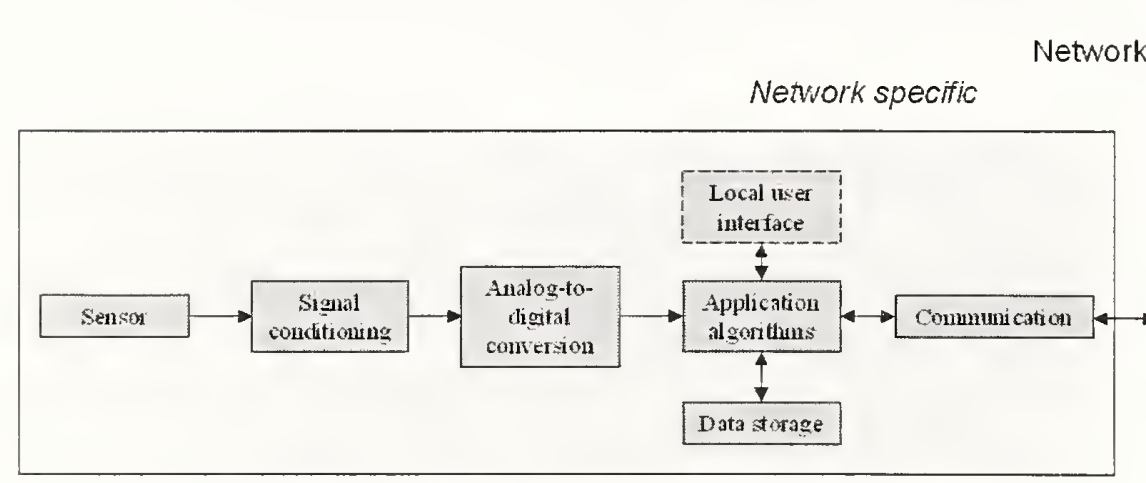

Some points regarding "smart":

- Moving intelligence closer to the point of measurement/control.

- Confluence of transducers, computation and communication towards comınon goal.

- Goal: make it cost effective to integrate/maintain distributed systems. 


\section{1 partition of general model}

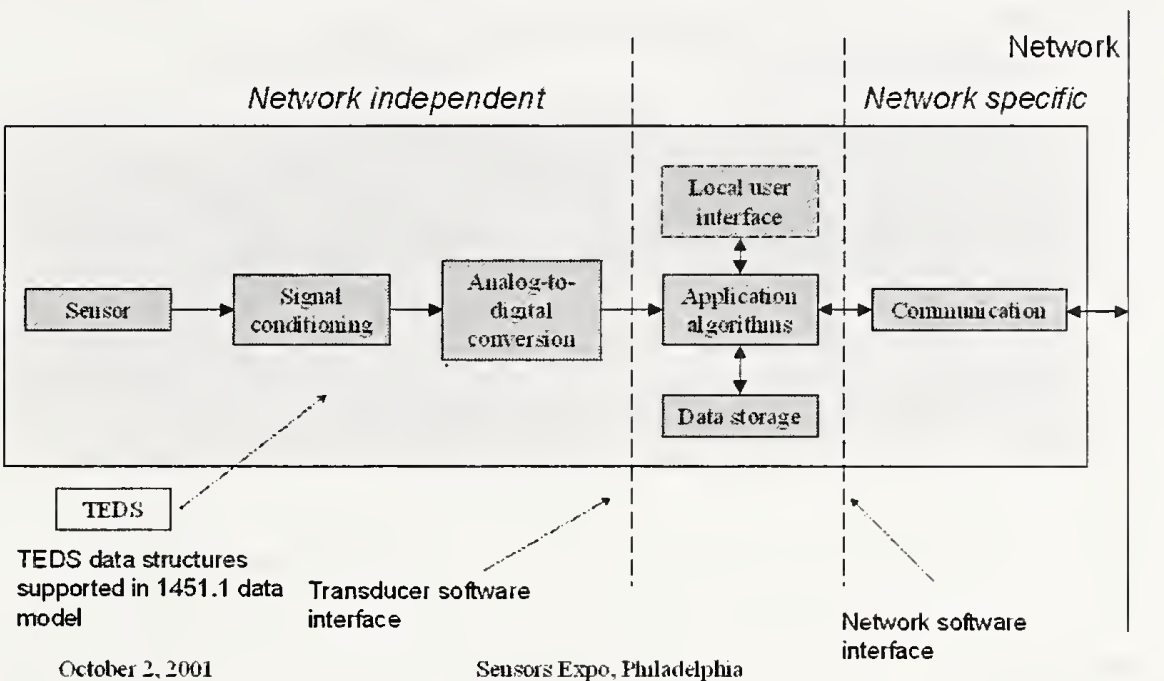

Octoler 2, 2001

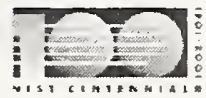

1451.1 resulting implementation

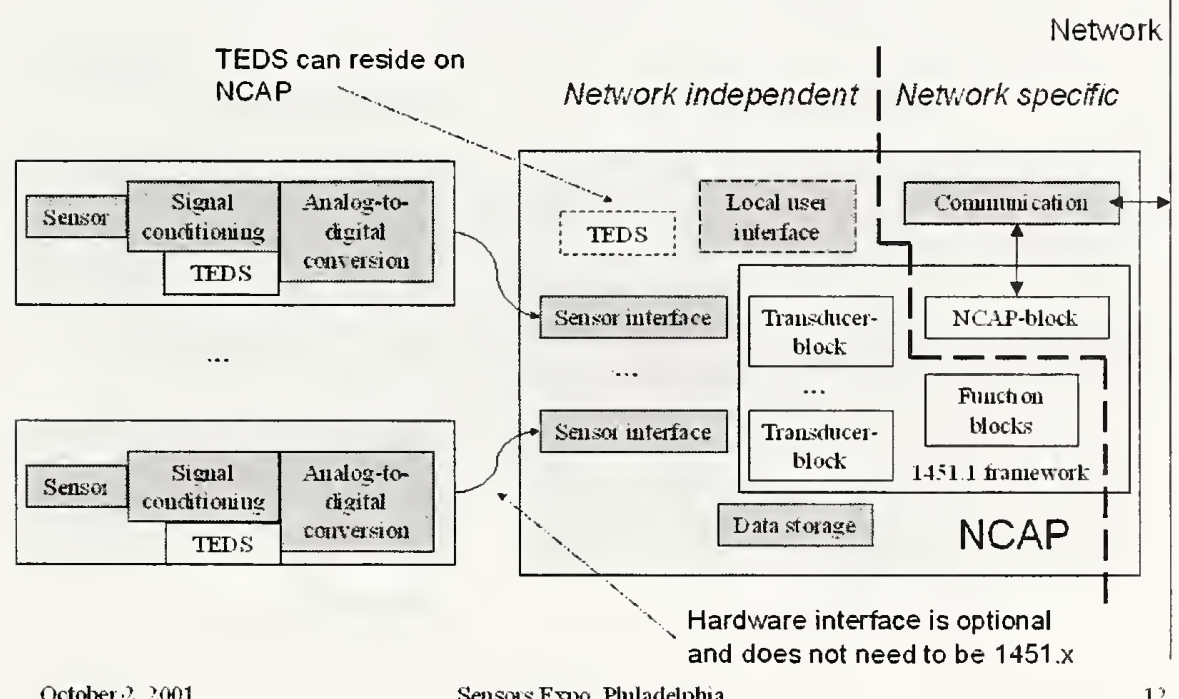




\section{IEEE Std 1451.1-1999 distinguishing features}

- Common object model can be used with multiple networking protocols.

- Uniform models for key functions needed in smart transducers including physical parametric data, application functionality and communication.

- Framework is defined to help create smart transducers.

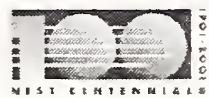

1451.2 partition of general model

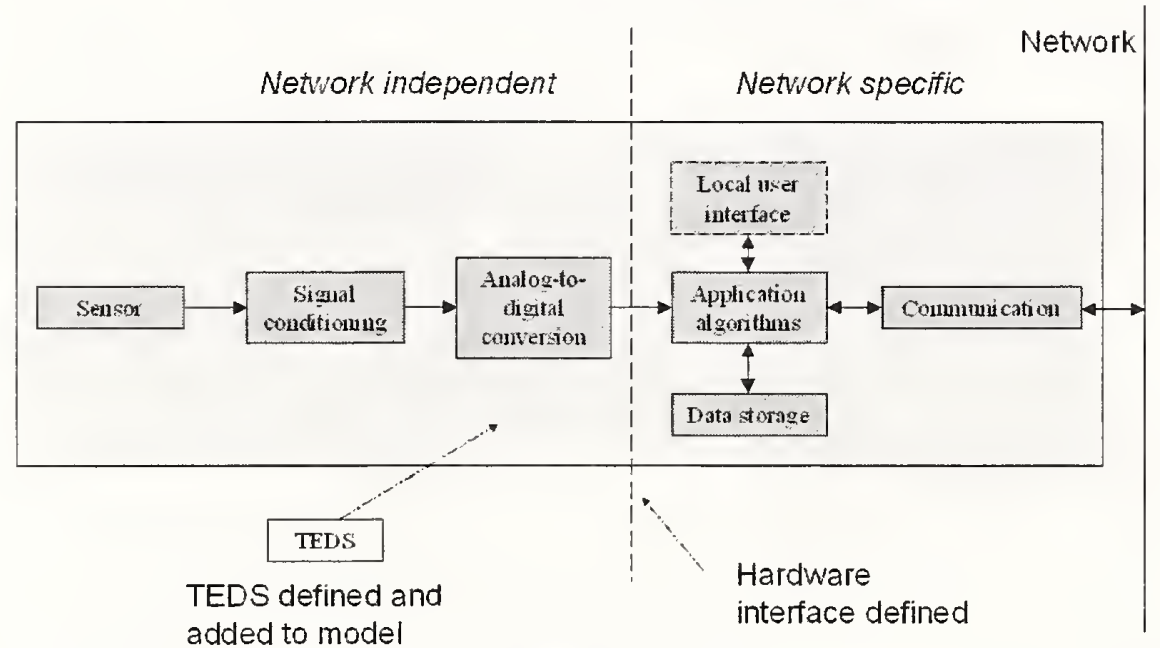




\section{2 resulting implementation}

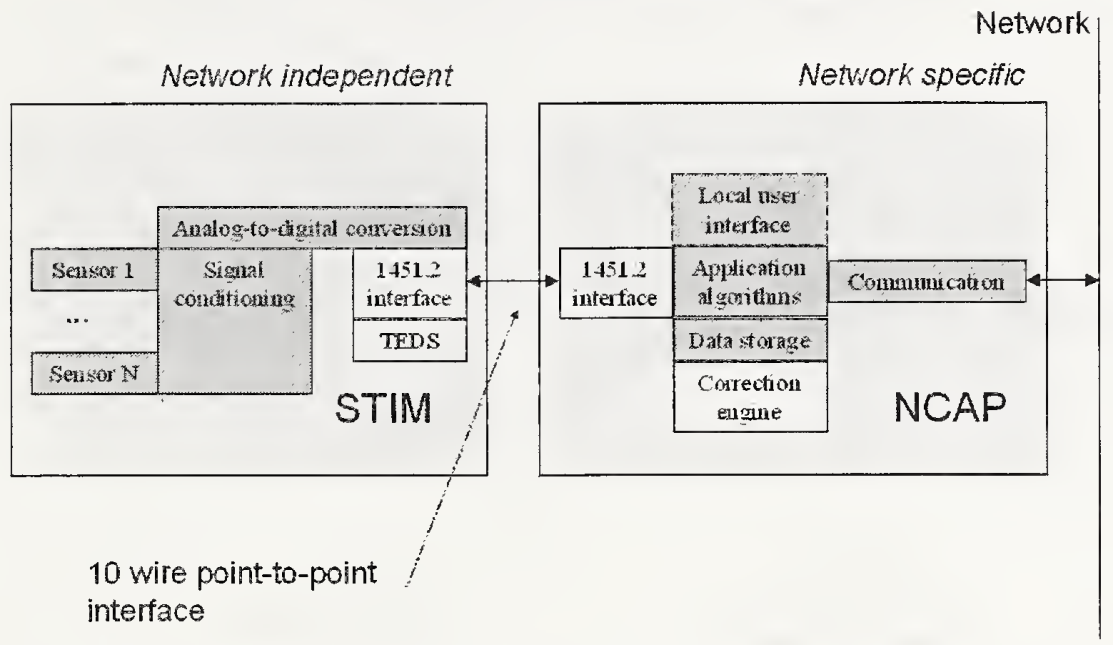

\section{IEEE Std 1451.2-1997 distinguishing features}

- Extensible Transducer Electronic Data Sheet (TEDS)

- General calibration/correction model for transducers.

- Physical units representation based on SI units.

- Triggering and control model defines how channels are accessed.

- All channels may be triggered simultaneously, timing parameters are used to indicate channel differences.

- Models for different kinds of sensors

- Powerful concept of correction engine and flexible location of correction engine. 


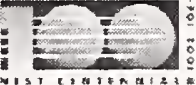

\section{P1451.3 partition of general model}

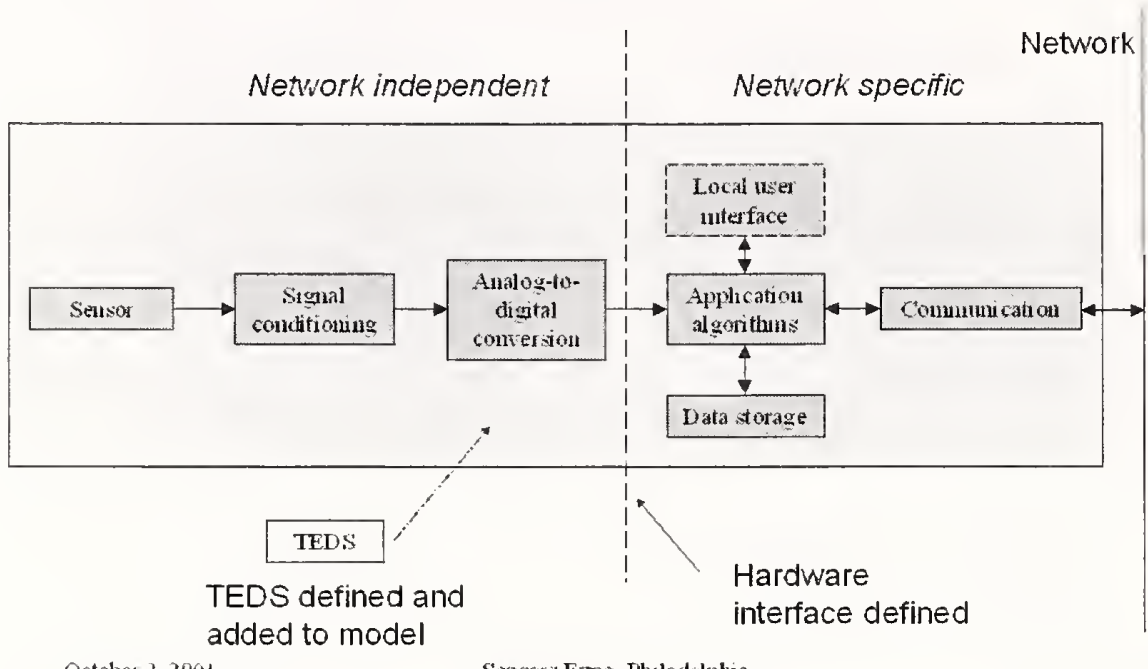

\section{P1451.3 resulting implementation}

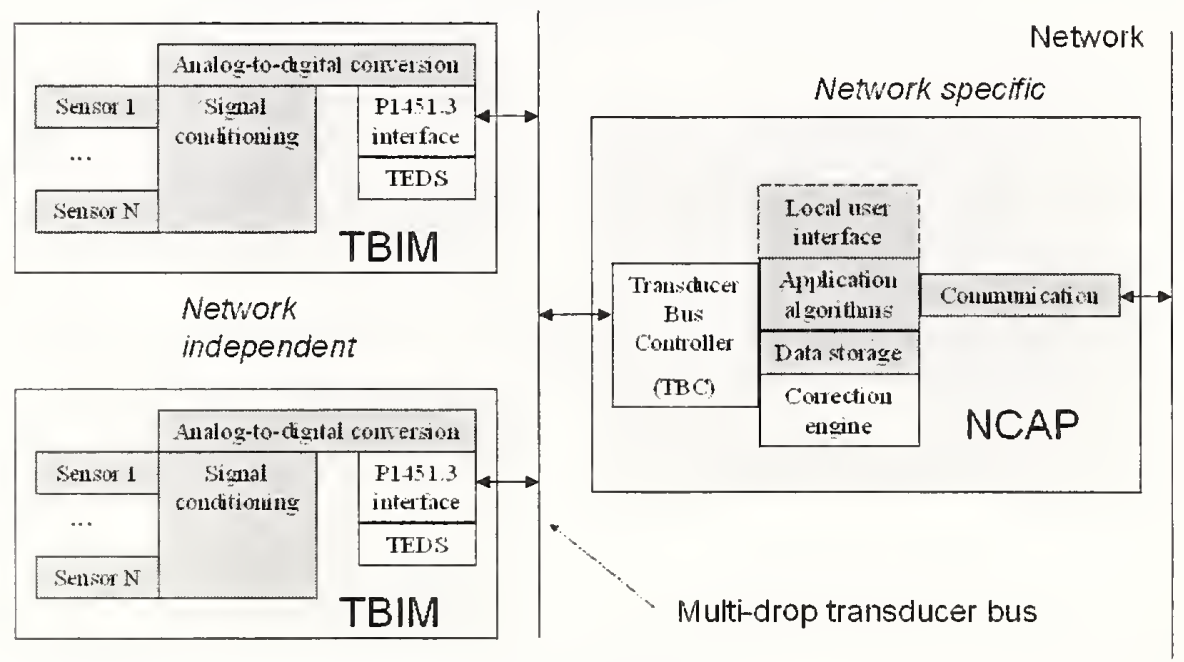




\section{IEEE P1451.3 distinguishing features}

- Multi-drop, high speed interface permits continuous streaming of data to host.

- Similar to 1451.2 in terms of TEDS, calibration/correction model, triggering/control model, data models.

- TEDS enhanced with new features such as XML format, more actuator models.

- Synchronized measurements at the Transducer Bus Interface Module (TBIM).

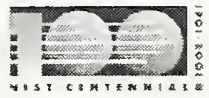

\section{P1451.4 partition of general model}

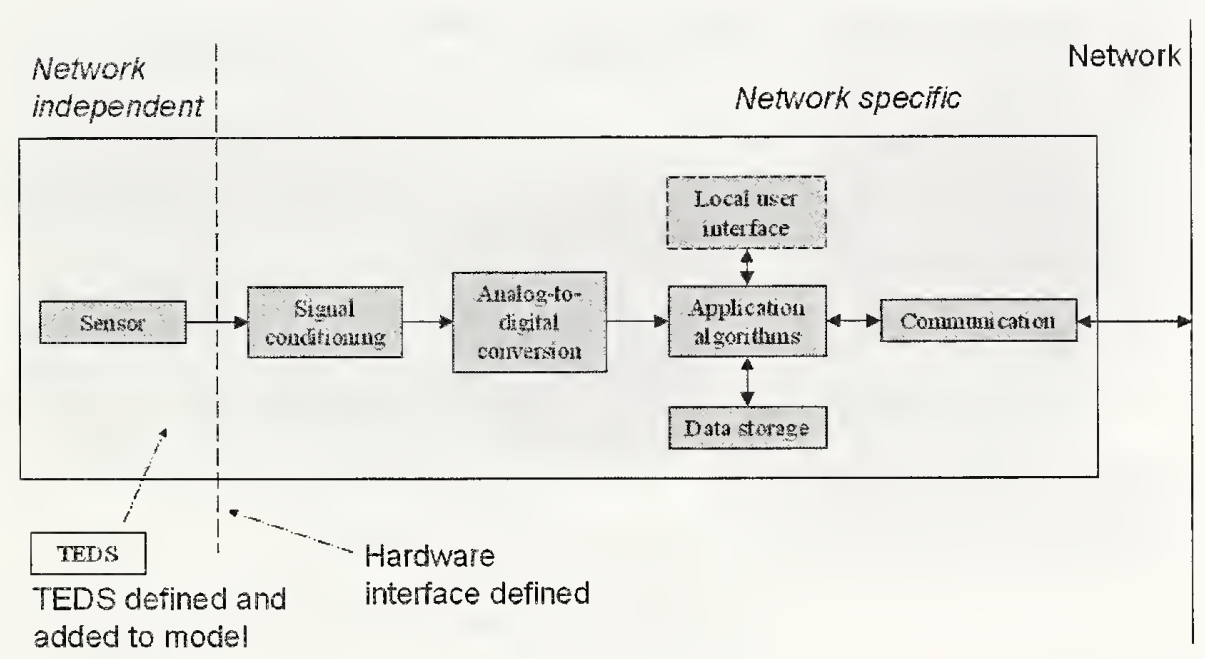

October 2. 2001 


\section{P1451.4 resulting implementation}

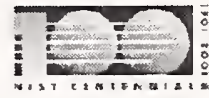

Network

Network

independent

Network specific

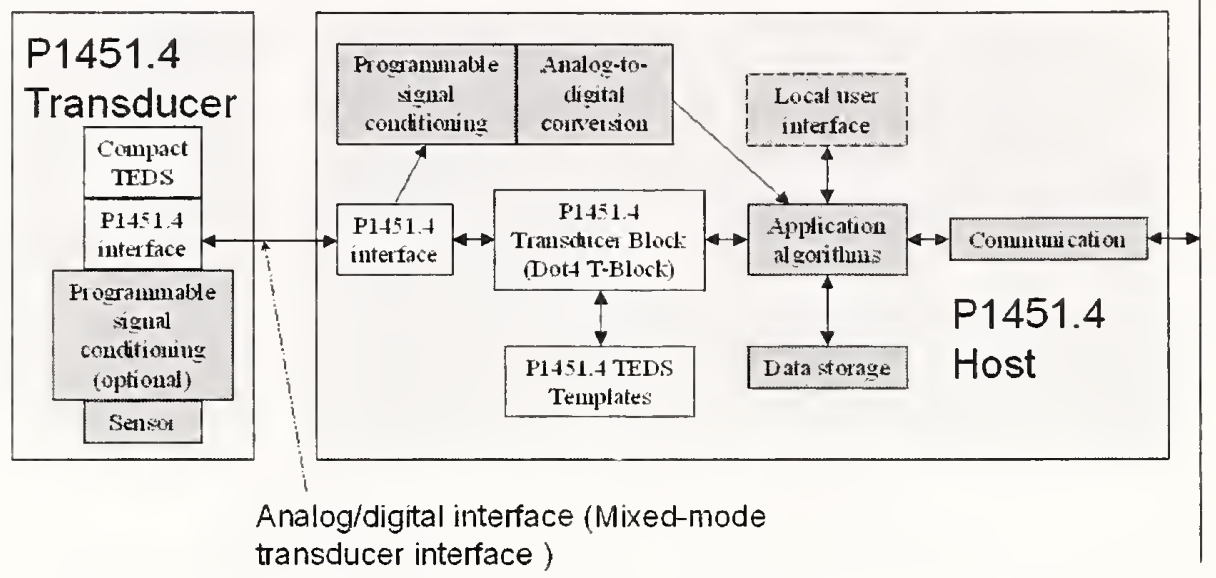

October 2. 2001

Seusor: Expo, Fluladelpha

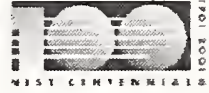

\section{Decoding a P1451.4 TEDS}

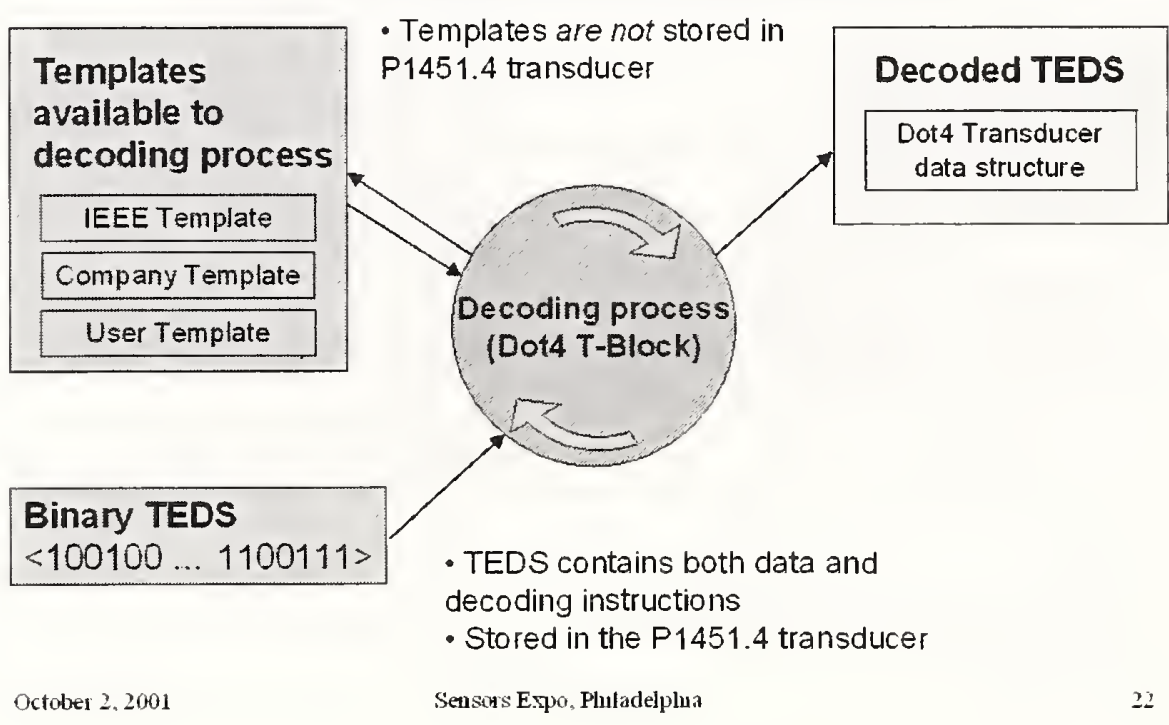




\section{IEEE P1451.4 distinguishing features}

- Compact TEDS is very small, sized in bits (as small as 67 bits, typically 256 bits), not bytes.

- TEDS plus Templates permits extensible self-identification of key transducer parameters.

- Mixed mode interface:

- Digital interface to read and write the TEDS or control the transducer. For example: adjust pre-amplifier gain, change filter setting. start self-test.

- Analog interface to make measurements in normal manner.

\section{Relationship between 1451 standards}

- The 1451 standards are being developed to work together, however they also stand on their own.

- 1451.1 may be used without any of the other $1451 . x$ hardware interface specifications.

- 1451.x interfaces may be used without 1451.1, however, a similar software framework that provides physical parametric data, application functionality and communications is required to fully utilize the 1451.x devices for network access.

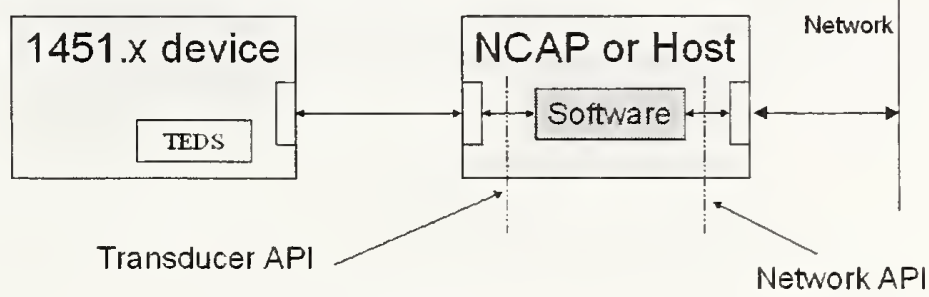




\section{Benefits from 1451}

\section{Sensor manufacturers}

- Multiple products may be developed just by changing the TEDS.

- Standard physical interfaces

- Standard calibration specification

\section{System integrators}

- Self-documenting hardware and software

- Systems that are easier to maintain

- Rapid transducer replacement

- Mechanism to store installation details

\section{Benefits from 1451 (continued)}

\section{Application software programmers}

- Standard transducer model for control and data

- Same model for accessing a wide variety of measurements

- "Hooks" for synchronization, exceptions, simultaneous sampling

- Support for multiple languages

\section{End users}

- Sensors that are easier to use; "you just plug them in".

- Analysis software that can automatically provide:

- physical units

- readings with significant digits

- transducer specifications

- installation details such as physical location and ID of transducer 


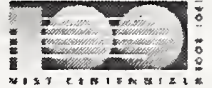

\section{For more information}

\begin{tabular}{l|l|l|l}
$\begin{array}{l}\text { 1451 } \\
\text { standard }\end{array}$ & Contact & Telephone & Email \\
\hline IEEE P1451 & Kang Lee & $301-975-6604$ & kang.lee@nist.gov \\
\hline IEEE 1451.1 & Jay Warrior & $650-485-2086$ & Jay_Warrior@agilent.com \\
\hline IEEE 1451.2 & Stan Woods & $650-485-5067$ & Stan_Woods@agilent.com \\
\hline IEEE P1451.3 & $\begin{array}{l}\text { Larry } \\
\text { Malchodi }\end{array}$ & $206-655-5695$ & larry.a.malchodi@boeing.com \\
\hline IEEE P1451.4 & Torben Licht & $\begin{array}{l}+4577412313 \\
\text { Denmark }\end{array}$ & TRLICHT@bk.dk \\
\hline
\end{tabular}

October 2, 2001

Sensors Expo, Fhuladelplua 


\subsection{Key Technical Features of IEEE Std 1451.2-1997}

\section{Key technical features of IEEE Std 1451.2-1997}

\section{Stan Woods}

\section{Agilent Technologies}

October 4, 2001

\section{A general model of a smart sensor}

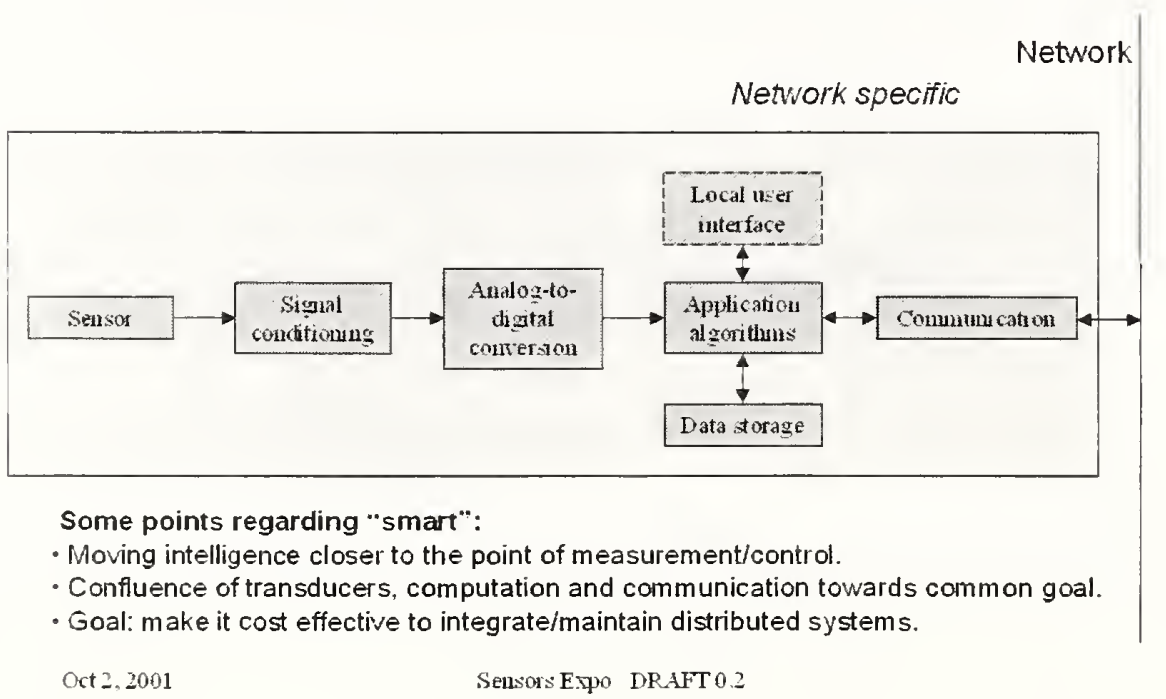




\section{2 partition}

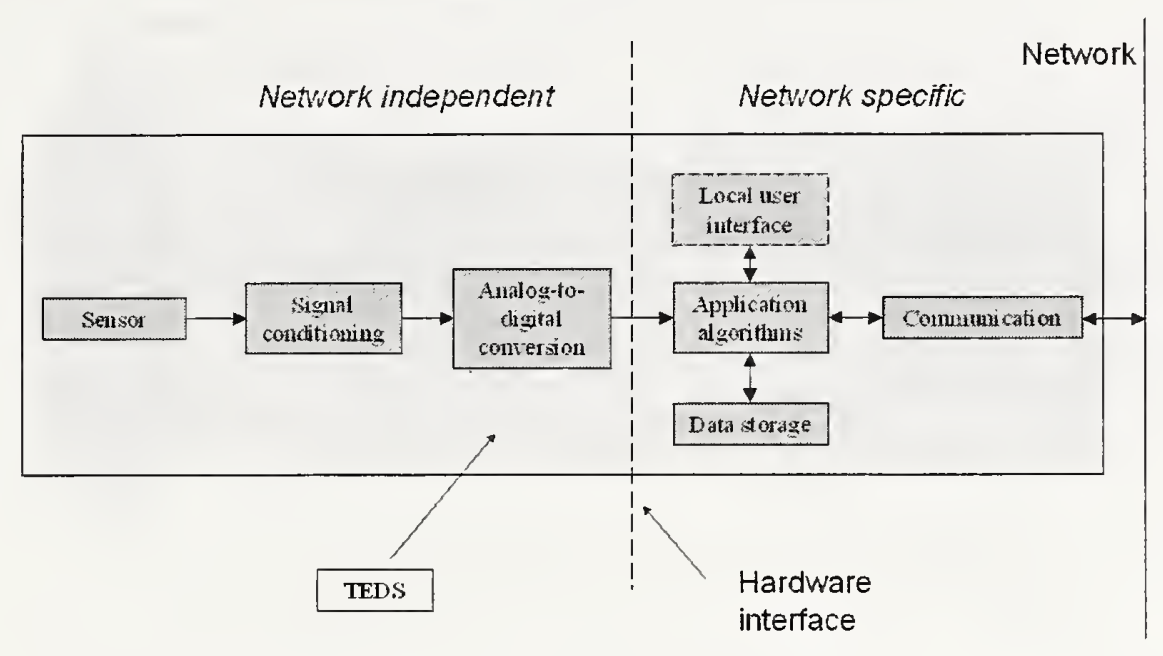

$\operatorname{Oct} 2,2001$

Sensor: Expo DRAFT 0.2

\section{IEEE Std 1451.2-1997 distinguishing features}

- Extensible Transducer Electronic Data Sheet (TEDS)

- General calibration/correction model for transducers.

- Physical units representation based on SI units.

- Triggering and control model defines how channels are accessed.

- All channels may be triggered simultaneously, timing parameters are used to indicate channel differences.

- Models for different kinds of sensors

- Powerful concept/location of correction engine allows flexibility in system design. 


\section{2 smart sensor model}

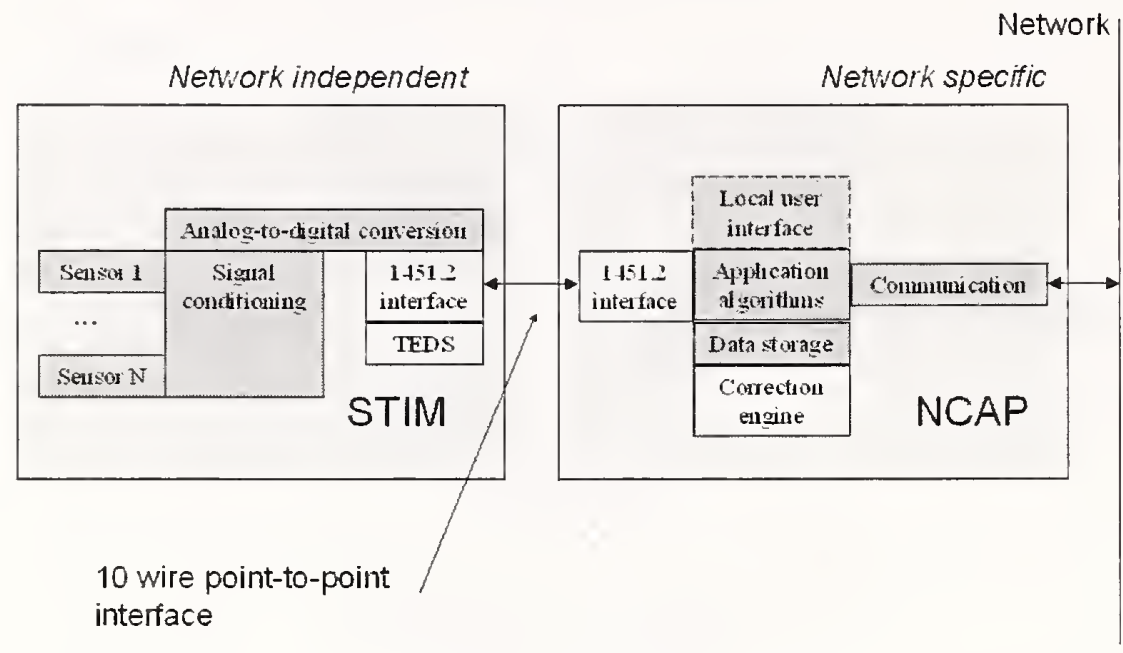

\section{2 hardware interface}

1) Communication

2) Triggering/handshaking

3) Interrupts and hot swap

4) Power

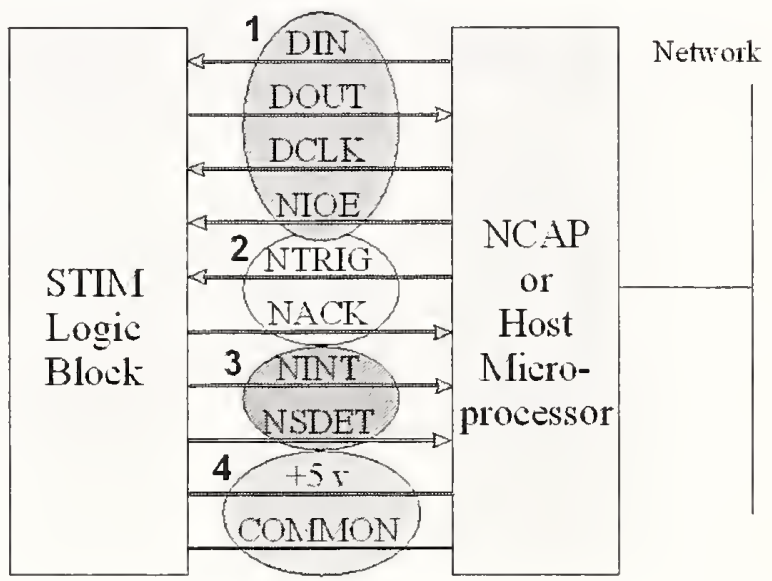

Sensets Expo DRAFT 0.2 


\section{STIM control/data model}

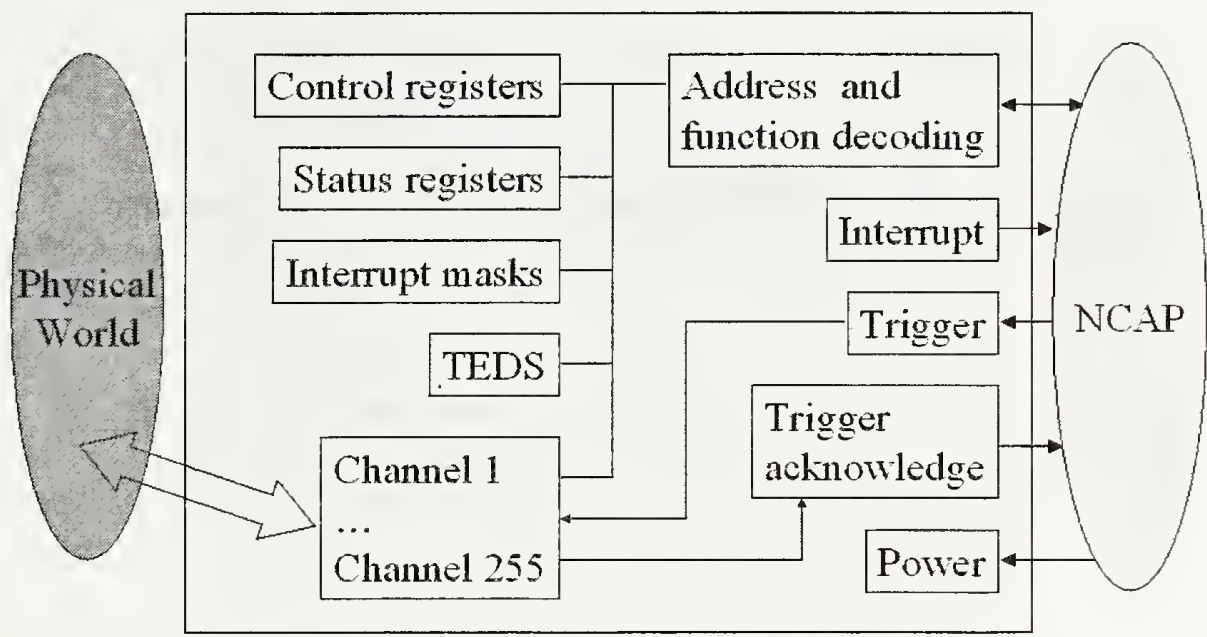

\subsection{TEDS blocks}

Machine readable

\begin{tabular}{|l|}
\hline $\begin{array}{l}\text { Meta-TEDS } \\
\text { (mandatory) }\end{array}$ \\
\begin{tabular}{|l} 
Channel \\
TEDS \\
(mandatory)
\end{tabular} \\
\hline $\begin{array}{l}\text { Calibration } \\
\text { TEDS }\end{array}$ \\
\hline
\end{tabular}

Human readable

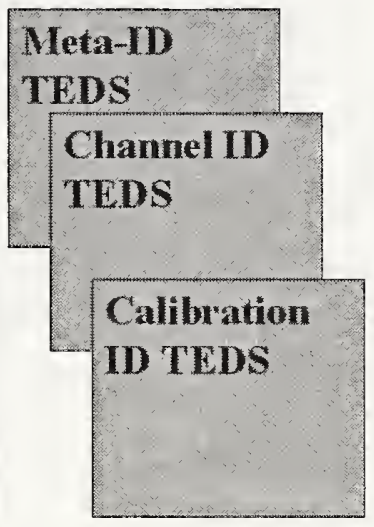

Sensors Expo DRAFT 0.2
Application specific

End Isers'
Application
specific
TEDS

Future extensions

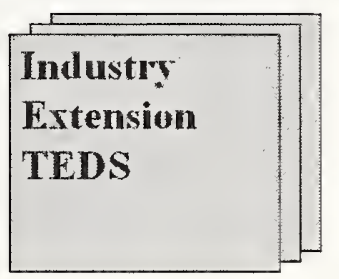




\section{System issues addressed by 1451.2 architecture}

\subsection{Architecture}

- Distance is achieved with the networli

- Plug and play at the transduce level with short distance interface (or hidden if NCAP and STIM are integrated).

- Last few feet achieved with analog wiring.

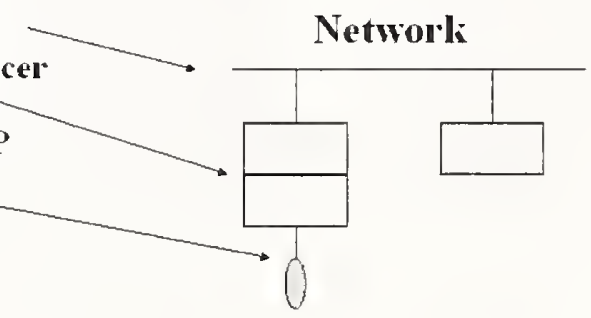




\section{Node Design Tradeoffs}

- Big NCAP and little STIMIs:

e g NCAPs with multiple 1451.2 ports

- Little NCAP and big STIMs:

e.g STIMs with many chamels

- Scalability

- type of network

- processing porrer

- type of processor

- number of channels

- types of sensors and actuators

- hardware interface speed

- connectors or PCB traces
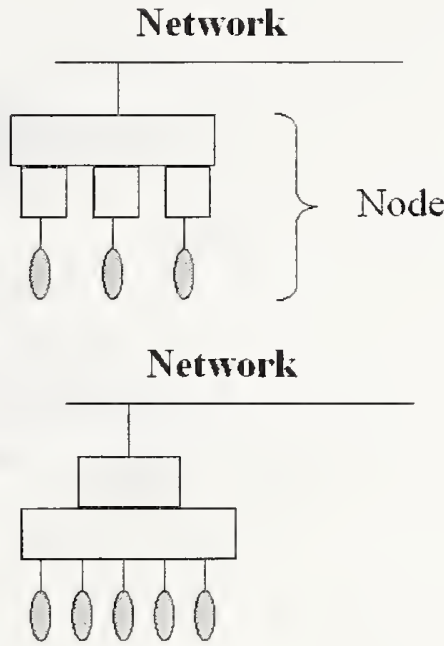

\section{System performance issues}

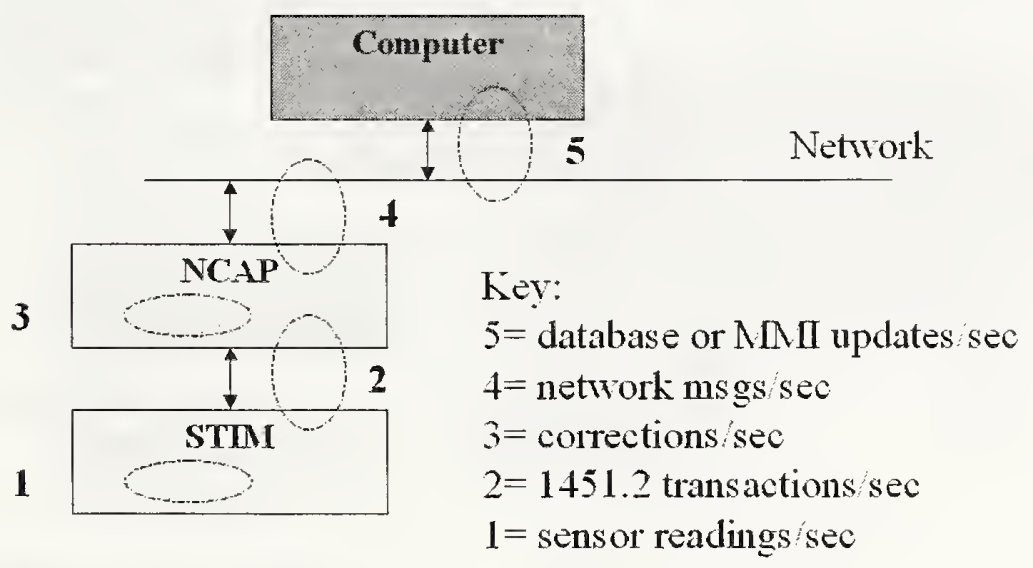




\section{2 correction engine in the NCAP}

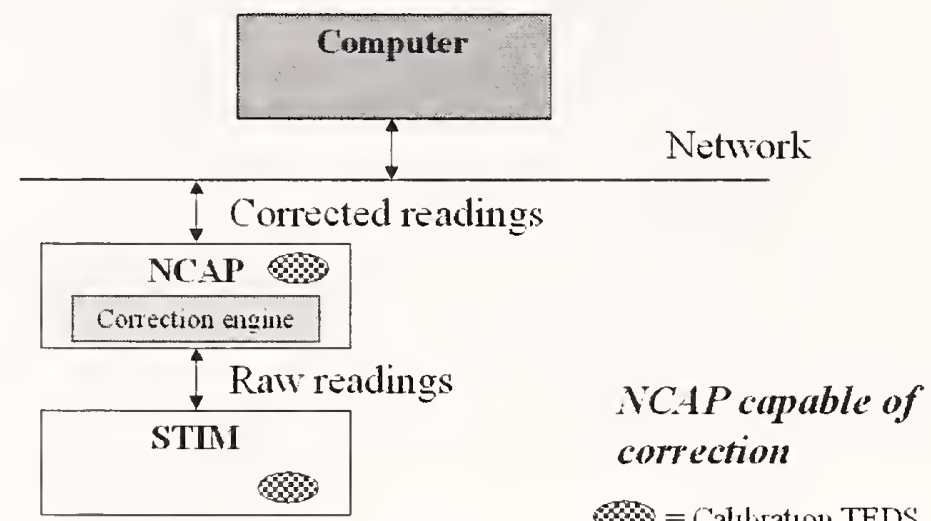

\section{$1+51.2$ correction engine "elsewhere" in the system}

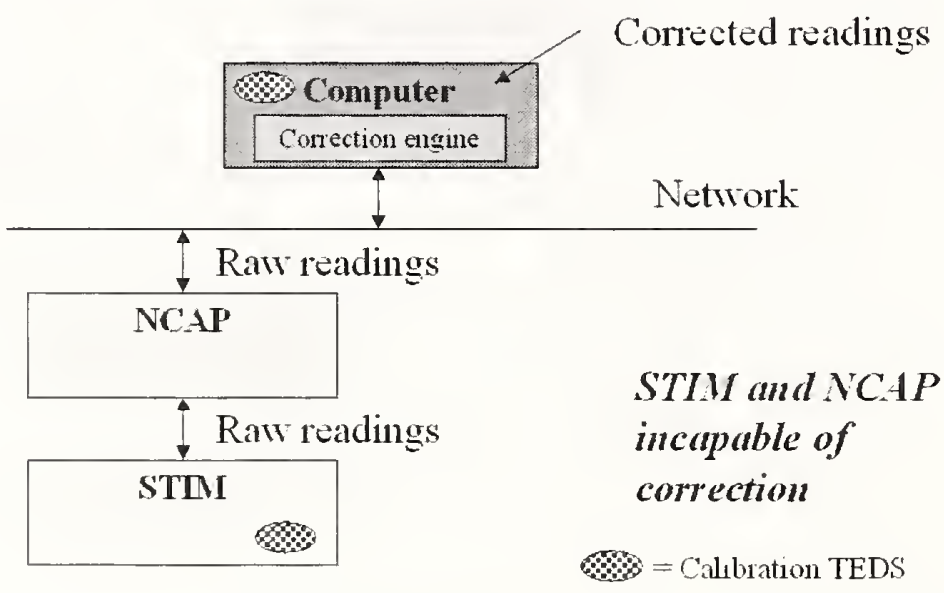




\section{2 correction engine in the STIM}

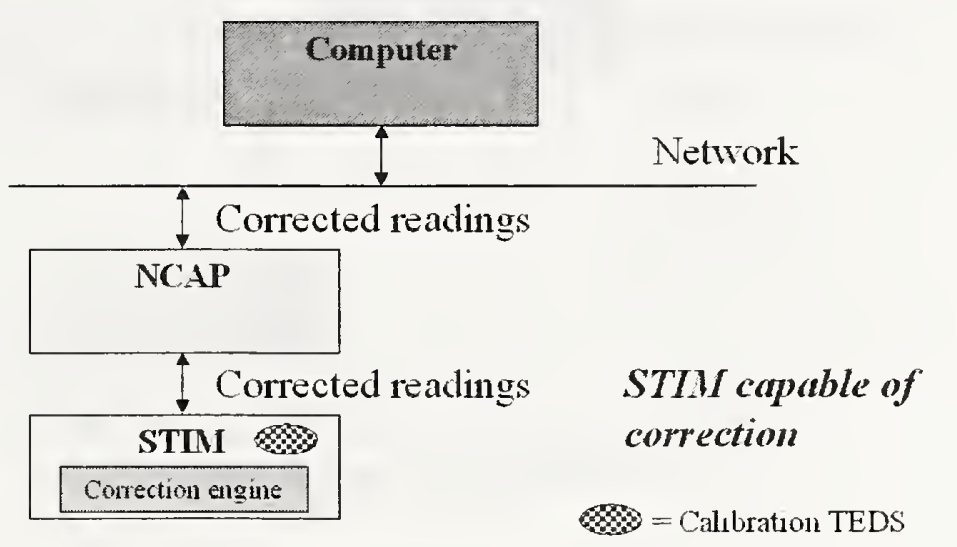

\section{Measurement/control loops}

- With the networli/ $1+51.2$ architecture there are three loops which may be used for measurement and control.

- 1) Conth ol by layers above the NCAP

- 2) NCAP-based control of STIM channels

- 3) Control done within a STIM

- Control may be:

- Client'server (poll set. 'pull', tightly coupled)

- Publish subsa ibe ('push'. loosely coupled)

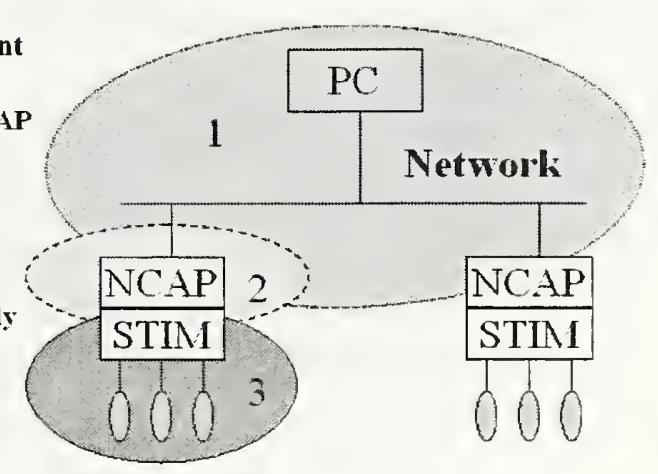




\section{Synchronization}

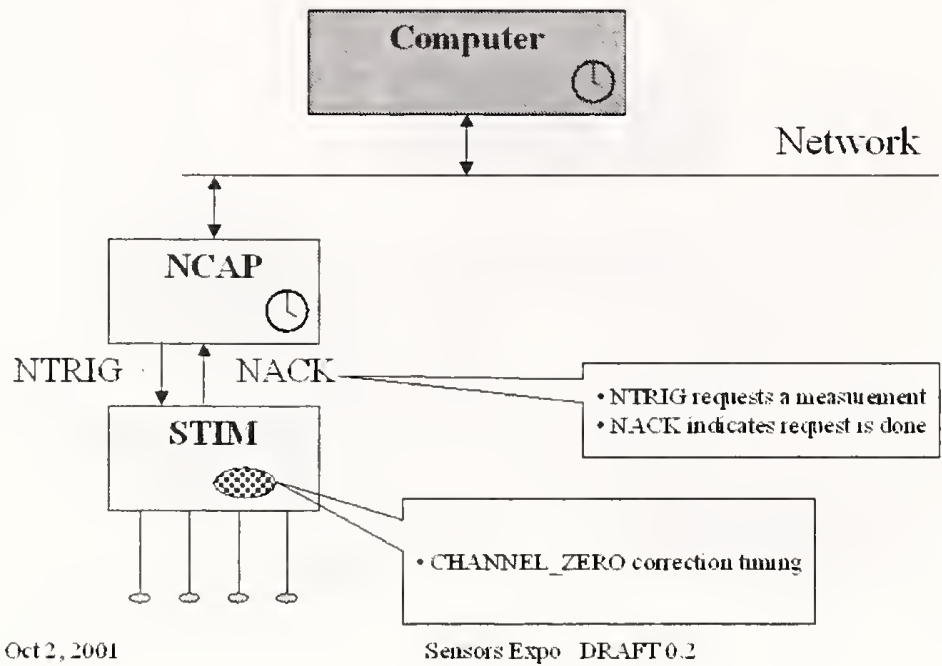

\section{Next steps}

- Consider changes to:

- TEDS

- Data/control model

- Hardware interface

- The following presentations will provide perspectives on the inplementation of 1451.2 and nore information on proposed changes. 


\title{
3.3 Proposed Enhancements to the IEEE 1451.2 Standard for Smart Transducers
}

\section{Proposed Enhancements to the IEEE 1451.2 Standard for Smart Transducers}

\author{
Robert N. Johnson, Telemonitor, Inc.
} Stan Woods, Agilent Technologies, Inc.

October 2, 2001

\section{Introduction}

- General benefits of smart sensors and of IEEE 1451.2 have been discussed in the previous presentations

- IEEE 1451.2-1997 was approved in September. 1997

- Defined terms and established basic principles of smart transducers and communications with transducers

- Received wide praise for technical accomplishments but has not enjoyed wide use

- Due for five-year review and vote on renewal next year

- This paper will discuss requested changes and proposed enhancements

- Workshop October 4, 2001 to discuss this in more detail

- Please attend the workshop! We want your feedback and support 


\section{What is a smart sensor?}

- Several definitions of smart sensor; we will use the one from IEEE 1451.2-1997

- A Smart Transducer is "A transducer that provides functions beyond those necessary for generating a correct representation of a sensed or controlled quantity. This functionality typically simplifies the integration of the transducer into applications in a networked environment."

- A Smart Sensor is "A sensor version of a smart transducer."

- Key concept: A smart sensor adds value to the data to enable or support distributed processing and decision making

\section{IEEE 1451.2 smart sensor model}

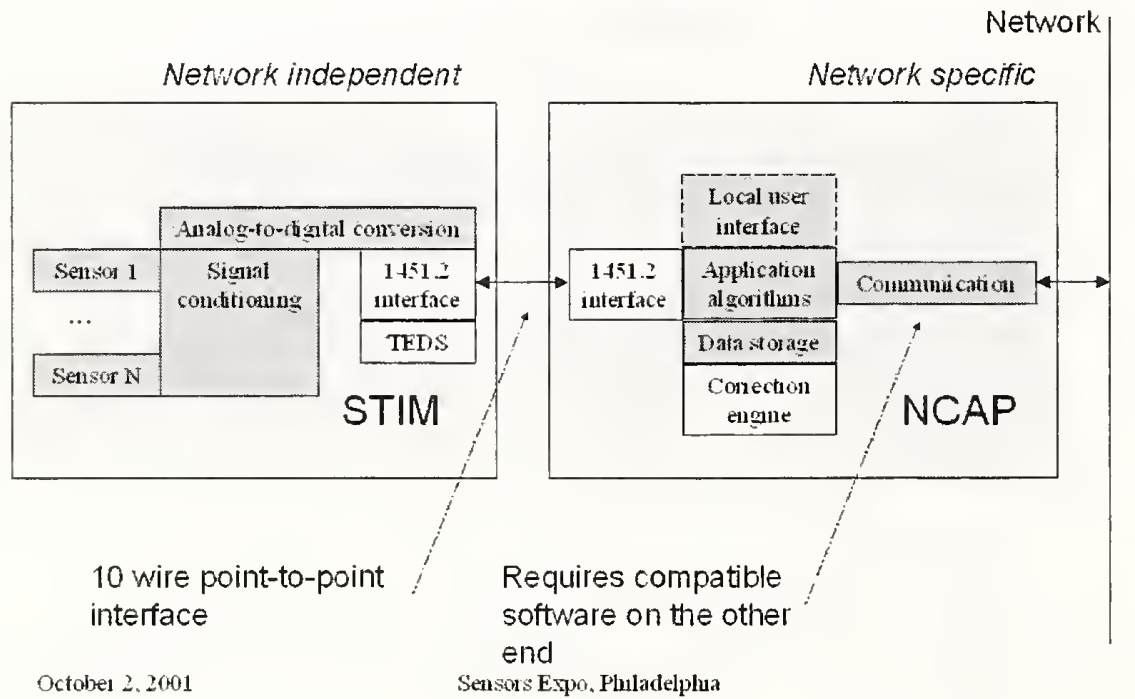




\section{IEEE 1451.2 hardware interface}

1) Communication/framing

2) Triggering/handshaking

3) Interrupts and hot swap

4) Power

Intended for closely -coupled systems
Network

STIM

Logic

Block-

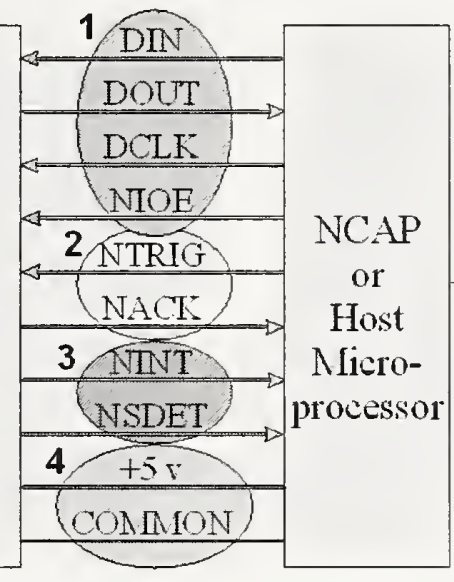

Netrork

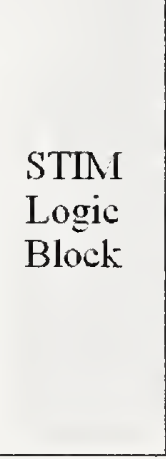

$\mathrm{NCAP}$

or

Host

Microprocessor

\section{IEEE 1451.2-1997 valuable features}

- Extensible Transducer Electronic Data Sheet (TEDS)

- General calibration/correction model for transducers

- Physical units representation based on SI units

- Triggering and control model defines how channels are accessed

- All channels may be triggered simultaneously, timing parameters are used to indicate channel differences

- Models for different kinds of sensors

- Powerful concept/location of correction engine allows flexibility in system design 


\section{IEEE 1451.2 TEDS blocks}

Machine readable

\begin{tabular}{|l|}
\hline $\begin{array}{l}\text { Meta-TEDS } \\
\text { (mandatory) }\end{array}$ \\
\begin{tabular}{|l} 
Channel \\
TEDS \\
inandatory)
\end{tabular} \\
Calibation \\
TEDS \\
\hline
\end{tabular}

October 2.2001
Human readable

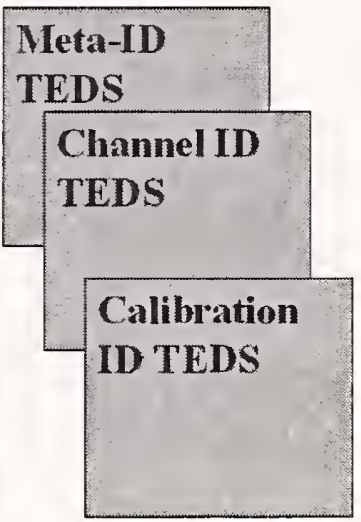

Seusors Expo, Fhuladelpha
Application specific

\begin{tabular}{|l|}
\hline End Users' \\
Application \\
specific \\
TEDS
\end{tabular}

Future extensions

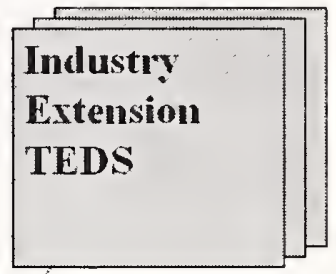

\section{Power of TEDS plus correction engine}

Multi-channel acceleration/tilt sensor:

- Hardware channels for temperature and acceleration

- Correction engine performs temperature compensation

- Virtual actuators for zero correction

- Virtual sensors for tilt (roll and pitch)

- Correction engine converts to angle

- Number order of channels is important

\begin{tabular}{|c|c|c|c|c|c|}
\hline \multicolumn{6}{|c|}{ Data Channels } \\
\hline No. & Propety & Type & Thits & Minimum & Maximum \\
\hline 1 & Temperature & Sensor & $\mathrm{K}$ & $23315\left(-40^{\circ} \mathrm{C}\right)$ & $358.15\left(85^{\circ} \mathrm{C}\right)$ \\
\hline 2 & Roll Zero & A.ctuator & radans & $-1.57\left(-90^{\circ}\right)$ & $1.57\left(90^{\circ}\right)$ \\
\hline 3 & Putch Zero & Actuator & radians & $-1.57\left(-90^{\circ}\right)$ & $1.57\left(90^{\circ}\right)$ \\
\hline 4 & Roll Zero & Sensor & radians & $-157\left(-90^{\circ}\right)$ & $157\left(90^{\circ}\right)$ \\
\hline 5 & Pitch Zero & Sensor & radians & $-1.57\left(-90^{\circ}\right)$ & $1.57\left(90^{\circ}\right)$ \\
\hline 6 & $\mathrm{X}$ Áccelaration & Sensor & $\mathrm{m} / \mathrm{s}^{2}$ & $-19.6(-2 g)$ & $196(2 \mathrm{~g})$ \\
\hline 7 & Y Acceleranon & Sensor & $\mathrm{m} / \mathrm{s}^{2}$ & $-196(-2 g)$ & $19.6(2 \varepsilon)$ \\
\hline 3 & Roll (ab out $\bar{X}$ ) & Sensor & rachans & $-1.31\left(-75^{\circ}\right)$ & $1.31\left(75^{\circ}\right)$ \\
\hline 9 & Pttch (about $\mathrm{Y}$ ) & Sensor & radians & $-1.31\left(-75^{\circ}\right)$ & $1.31\left(75^{\circ}\right)$ \\
\hline
\end{tabular}

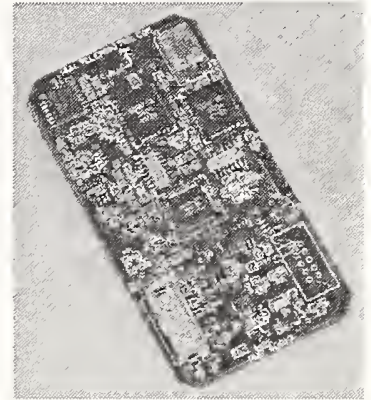




\section{Most requested changes to IEEE 1451.2}

- Make it easier to understand and implement

- Make the hardware interface faster

- Use less wires

- Pick a standard connector

- Provide for electrical isolation

- Allow real-time reconfiguration

- Add frequency response to TEDS and correction engine

- Make NCAPs readily available and compatible with existing systems

- Don't add unnecessary expense to simple transducers

- Add security, timestamps, data logging, etc.

\section{Most requested changes, con't.}

- Make it easier to understand and implement

- New, broad-reaching standard

- Wide adoption will produce user guides, books, etc.

- Not everyone must understand the details

- OEMs can convert raw transducers to STIMs without having to design signal conditioning and conversion

- Make the hardware interface faster

- Minimum of 6.000 bits supports inexpensive hardware

- Maximum not specified: several million bit/s has been demonstrated

- Digital interface may not be appropriate for all applications (e.g. IEEE P1451.4) 


\section{Most requested changes, con't.}

- Use less wires

- Originally for single close-coupled transducer to microprocessor

- Based on SPI with hardware handshaking

- Supports synchronized trigger and data acquisition

- Simpler interfaces are appropriate for some uses

- Pick a standard connector

- Connectors are very application-dependent

- Started defining connectors for some applications

- Alternate physical layers may include connectors

- True "plug-and-play" requires connector definition

\section{Most requested changes, con't.}

- Provide for electrical isolation

- Isolation less of an issue for close-coupled system

- Using existing standard physical layers will help

- Allow real-time reconfiguration

- STIM cannot tell NCAP that the TEDS has changed

- No standard mechanism for selectable gain, sample rate, number of samples, etc.

- Add frequency response to TEDS and correction engine

- Standard provides for extensions to TEDS but not to correction engine 


\section{Most requested changes, con't.}

- Make NCAPs readily available and compatible with existing systems

- Standard visualizes complete system including STIMS, NCAPs, network-level software

- Need growth path to bring benefits of IEEE 1451 to existing systems

- Don't add unnecessary expense to simple transducers

- Target is applications where interchangeable communicating smart transducers add value

- Some applications are too cost-sensitive

- Line between the two will shift over time

\section{Most requested changes, con't.}

- Add security, timestamps, data logging. etc.

- Originally viewed as higher-level functions

- Appropriate to consider for proposed enhancements

- Will depend on the interests of the people who participate in the working group

- Must avoid "rampant featuritis!" 


\section{Proposed enhancements to IEEE 1451.2}

- Primary enhancements:

- Partition the TEDS

- Alternative physical layers

- Partition the standard

- Secondary enhancements:

- Enhance the TEDS

- Add functions

- Standalone function

- Corrections and additions

\section{Partition the TEDS}

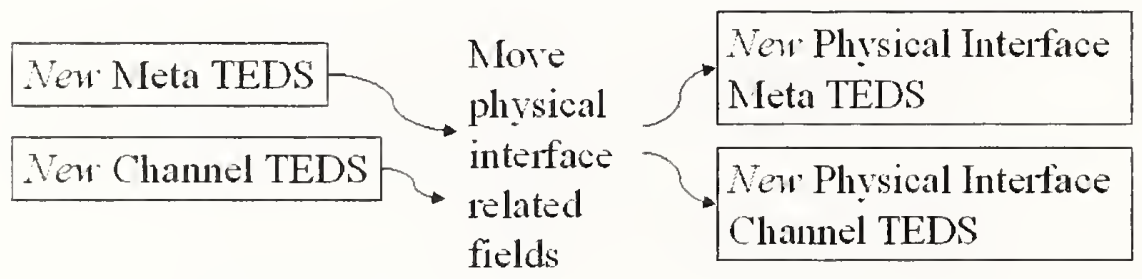

Meta-ID TEDS

Channel-ID TEDS 


\section{Proposed new IEEE 1451.2 TEDS blocks}

Machine readable

\begin{tabular}{|l|}
\hline $\begin{array}{l}\text { Meta-TEDS } \\
\text { (mandatory) }\end{array}$ \\
\begin{tabular}{|l|} 
Channel TEDS \\
(mandatory) \\
\hline Calibration TEDS \\
\hline Physical layer \\
Meta-TEDS \\
Physical layer \\
Channel-TEDS
\end{tabular} \\
\hline
\end{tabular}

October 2. 2001
Human readable

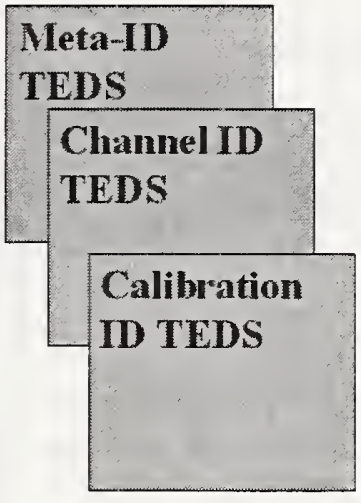

Application specific

End Users' Application specific TEDS

Future extensions

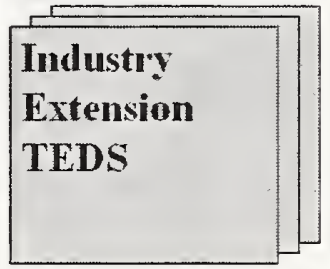

\section{Alternate physical layers}

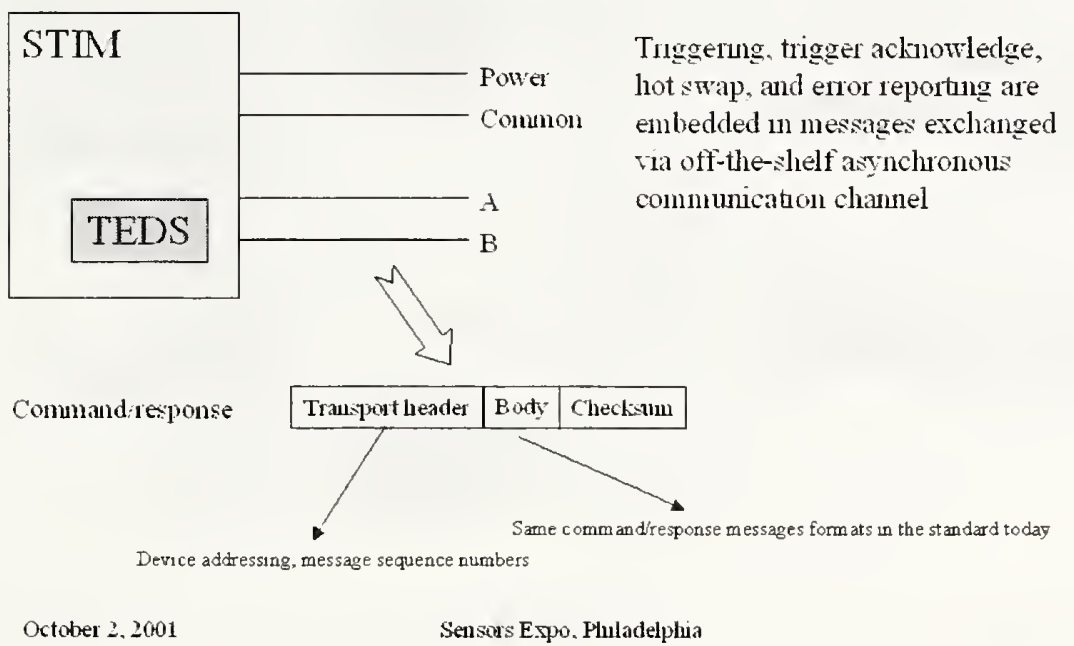




\section{Proposed serial version of IEEE 1451.2}

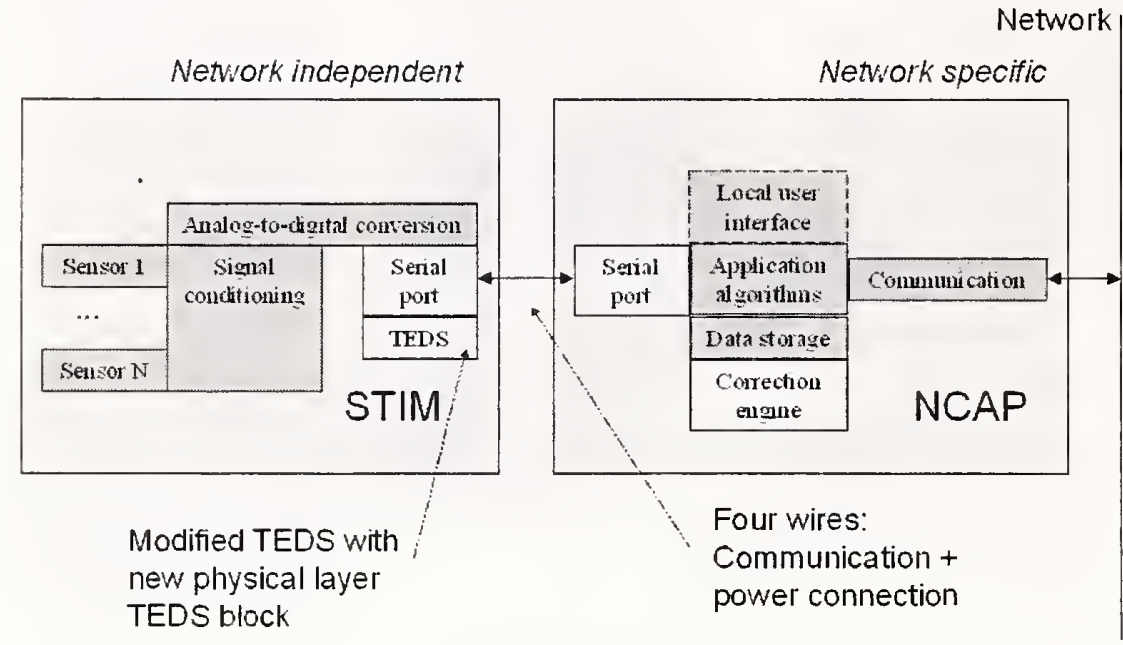

\section{Proposed IEEE 1451.2 serial interface}

1) Communication, triggering, error reporting 2) Power

Signal names for 1) will depend on particular physical layer

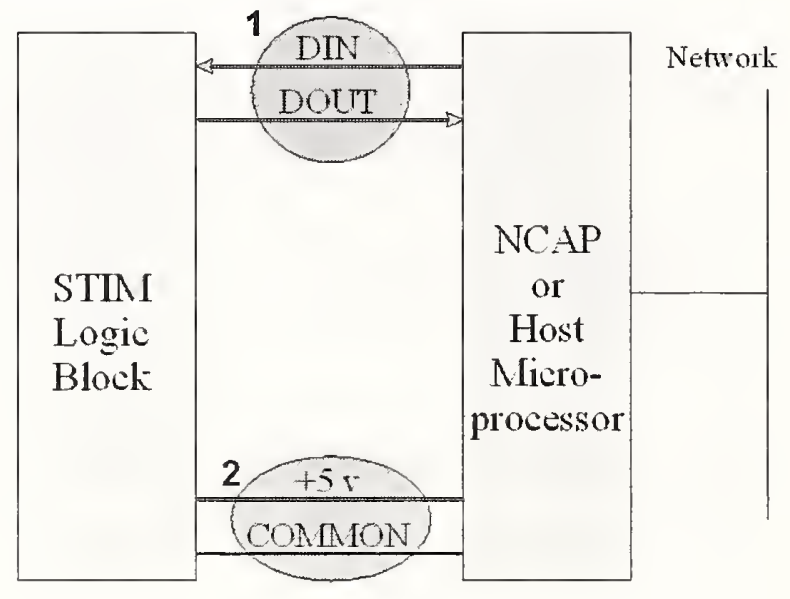

Sensors Expo. Fuladelphia

20 


\section{Support for serial IEEE 1451.2}

\section{Trends in UART support:}

- present on most microprocessors

- chips have become smaller, less expensive, more robust

- multi-port UARTs

- supported in ADI Microconverter family

Where can we plug into serial ports?

- Instruments

- 1/O cards

- Computers

- "Slot O" controllers in VME, VXI, CPCI, PXI card cages

- Handhelds, PDAs

\section{Where to put the STIM interface electronics?}
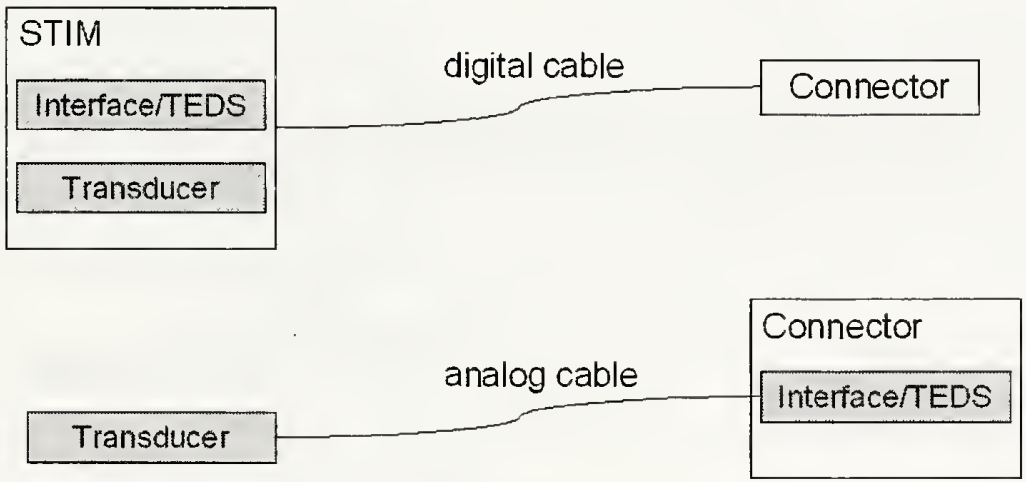


\section{Partition the Standard}

- Organize the standard around the OSI information model

- Separate sections for major functions:

- TEDS

- Correction engine

- Physical layer

\section{Partition the standard (allow TEDS only)}

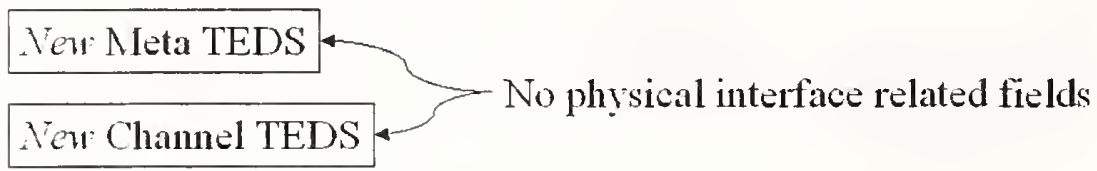

M.Ieta-ID TEDS

Channel-ID TEDS
Other TEDS blocks can remain the same. 


\section{Secondary enhancements}

- Enhance the TEDS

- Add features from IEEE P1451.3 and IEEE P1451.4

- Bandwidth

- Frequency response

- XML format

- Etc.

- Add functions

- Control function to tell NCAP to reload TEDS

- Support STIM reconfiguration:

- Gain, bandwidth, etc.

- Changes in channels due to hot-swap in local sub-net, including IEEE P1451.3 or IEEE P1451.4

\section{Secondary enhancements, con't.}

- Standalone function

- Support use with existing data and control systems

- Map IEEE 1451.2 functions to existing protocols:
- Modbus RTU
- Modbus
- ProfiBus
- HTTP URL-based
- XML
- Etc.

- Corrections and additions

- Miscellaneous comments received since publication

- Others identified during review and updating process 


\section{Alternative communication protocols}

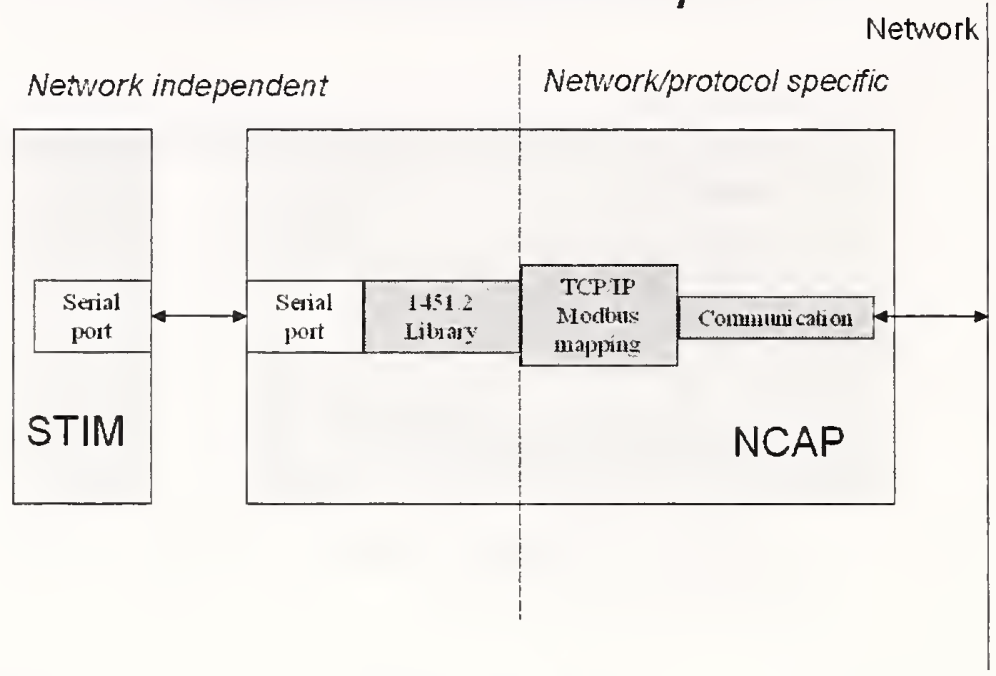

\section{New connectivity enabled by enhancements}

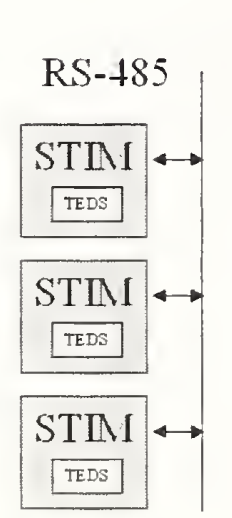

October 2, 2001

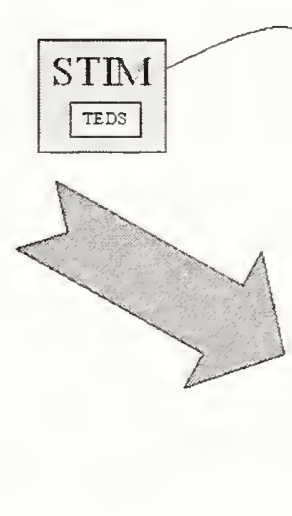

Comnectivity to existing communication ports:

- Computers

- Programmable logic controllers

- PC plug-1ll cards

- Instruments

- NCAP's 


\section{Applications for serial IEEE 1451.2}

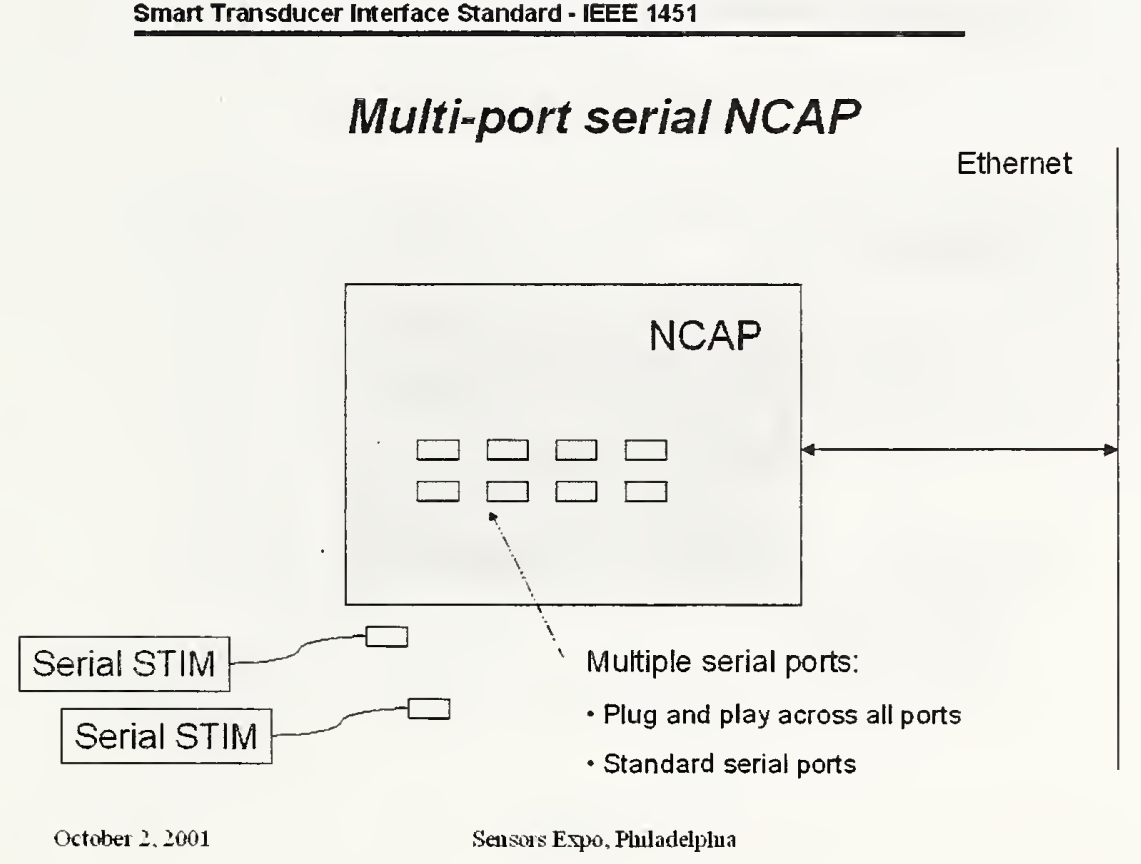




\section{Industrial card cage I/O card}

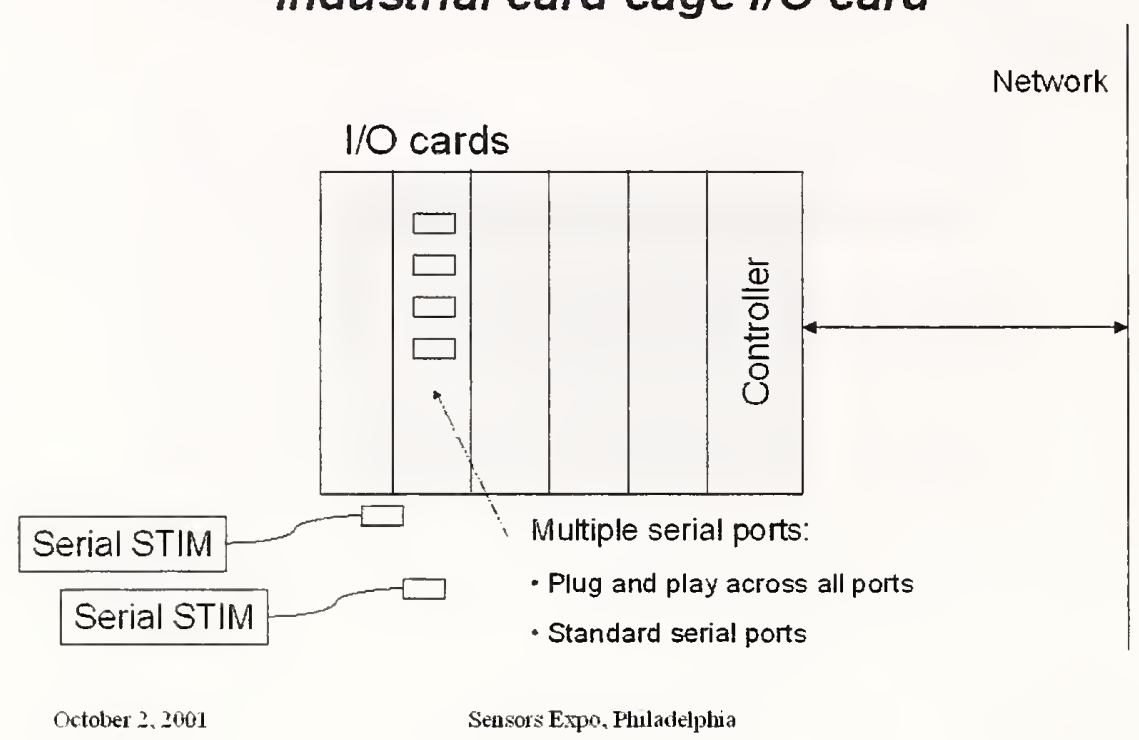

31

Smart Transducer Int erface Standard - IEEE 1451

\section{Computers and PDAs}

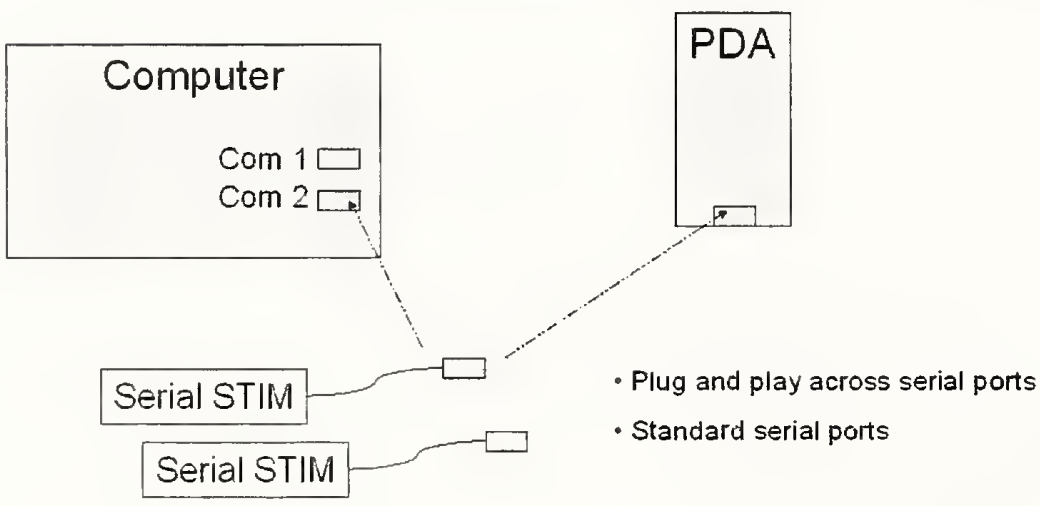




\section{Most requested changes to IEEE 1451.2}

- Make it easier to understand and implement

- Make the hardware interface faster

- Use less wires

- Pick a standard connector

- Provide for electrical isolation

- Allow real-time reconfiguration

- Add frequency response to TEDS and correction engine

- Make NCAPs readily available and compatible with existing systems

- Don't add unnecessary expense to simple transducers

- Add security, timestamps, data logging, etc.

\section{Conclusions}

- IEEE 1451.2 established several valuable basic principles of smart transducers

- The most important of these is the TEDS

- We need to keep the best parts of the original standard while addressing the current needs of the marketplace

- The proposed enhancements will address the major requested changes and will result in:

- More flexibility

- Lower cost

- Improved connectivity

- We need user comments and feedback on the proposed enhancements 


\section{Please attend the IEEE 1451.2 worlishop on October $t, 2001$ !}




\subsection{Requested Changes to the IEEE 1451.2 Standard for Smart Transducers}

\section{Requested Changes to the IEEE 1451.2 Standard for Smart Transducers}

Robert N. Johnson, Telemonitor, Inc. Stan Woods, Agilent Technologies, Inc.

October 4, 2001

\section{Most requested changes to IEEE 1451.2}

- Make it easier to understand and implement

- Make the hardware interface faster

- Use less wires

- Pick a standard connector

- Provide for electrical isolation

- Allow real-time reconfiguration

- Add frequency response to TEDS and correction engine

- Make NCAPs readily available and compatible with existing systems

- Don't add unnecessary expense to simple transducers

- Add security, timestamps, data logging, etc. 


\section{Most requested changes, con't.}

- Make it easier to understand and implement

- New, broad-reaching standard

- Wide adoption will produce user guides, books, etc.

- Not everyone must understand the details

- OEMs can convert raw transducers to STIMs without having to design signal conditioning and conversion

- Make the hardware interface faster

- Minimum of 6,000 bit/s supports inexpensive hardware

- Maximum not specified; several million bit/s has been demonstrated

- Digital interface may not be appropriate for all applications (e.g. IEEE P1451.4)

\section{Most requested changes, con't.}

- Use less wires

- Originally for single close-coupled transducer to microprocessor

- Based on SPI with hardware handshaking

- Supports synchronized trigger and data acquisition

- Simpler interfaces are appropriate for some uses

- Pick a standard connector

- Connectors are very application-dependent

- Started defining connectors for some applications

- Alternate physical layers may include connectors

- True "plug-and-play" requires connector definition 


\section{Most requested changes, con't.}

- Provide for electrical isolation

- Isolation less of an issue for close-coupled system

- Using existing standard physical layers will help

- Allow real-time reconfiguration

- STIM cannot tell NCAP that the TEDS has changed

- No standard mechanism for selectable gain, sample rate, number of samples, etc.

- Add frequency response to TEDS and correction engine

- Standard provides for extensions to TEDS but not to correction engine

\section{Most requested changes, con't.}

- Make NCAPs readily available and compatible with existing systems

- Standard visualizes complete system including STIIMS. NCAPs, network-level software

- Need growth path to bring benefits of IEEE 1451 to existing systems

- Don't add unnecessary expense to simple transducers

- Target is applications where interchangeable communicating smart transducers add value

- Some applications are too cost-sensitive

- Line between the two will shift over time 
Smart Transducer Interface Standard - IEEE 1451

\section{Most requested changes, con't.}

- Add security, timestamps, data logging, etc.

- Originally viewed as higher-level functions

- Appropriate to consider for proposed enhancements

- Will depend on the interests of the people who participate in the working group

- Must avoid "rampant featuritis!" 


\title{
3.5 A Sensor Manufacturer's Perspective
}

\section{A Sensor Manufacturer's Perspective}

True confessions of a $\mathbf{1 4 5 1 . 2}$ Pioneer

\author{
Jim Moore \\ Telemonitor, Inc
}

\section{A Generic Differential Pressure Transmitter}




\section{The Pressure Transmitter market}

- 1.+ Million units/year with $4.3 \%$ CAGR

- ARC Advisory Gr oup, Inc dita

- Manufacturers are vertically integrated

- Need > 50K/year to be profitable

- Moore Products developed the MycroSensor to compete in this marliet

- 1985 started MycroSensor development

- 1993 introduced the XTC Pressure transmitter

- However.....

\section{But If.........}

Ve could rewrite the transmitter business model:

- Multiple vendor's of pressure sensing STIMS

- Capacitive such as MycroSensor

- Piezoresistive

- NCAPS (transmitters) manufactured for targeted markets

$-\downarrow-20 \mathrm{~m}$-HART $(90 \%$ of market $)$

- Profibus, FF, etc

- Ethernet - future? 


\section{A 1451 Differential Pressure Transmitter}

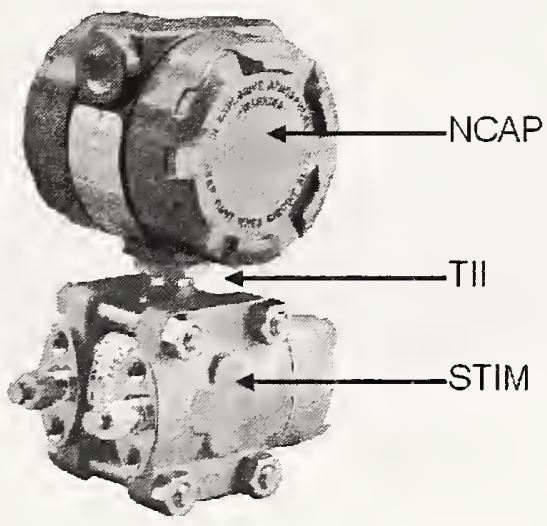

\section{New Applications}

- Remote nonitoring

- Maintenance

- Environnental

- Security, both defensive and offensive

- Gas flow measurement in the "Oil Patch" (an exanıle)

- Approximately 50K units per year

- Requires accurate measurement of 2 pressures and temperature.

- Low power requirements

- Replace old circular chant recorders 


\section{Ethernet MV Pressure transmitter}

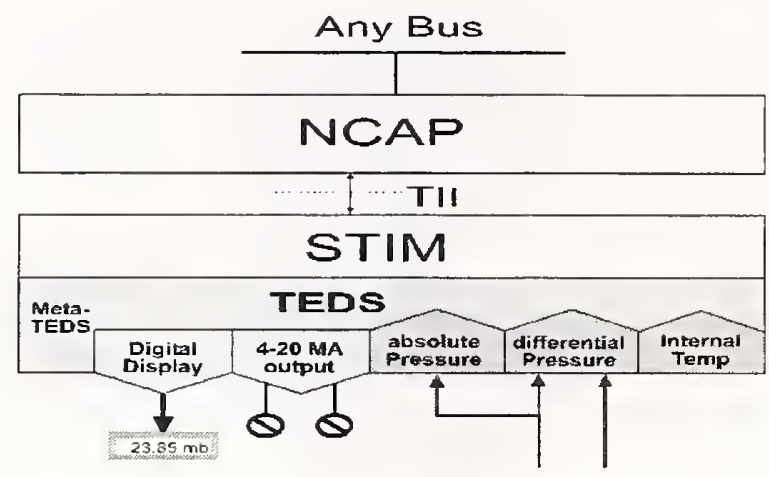

\section{The MycroSensor}

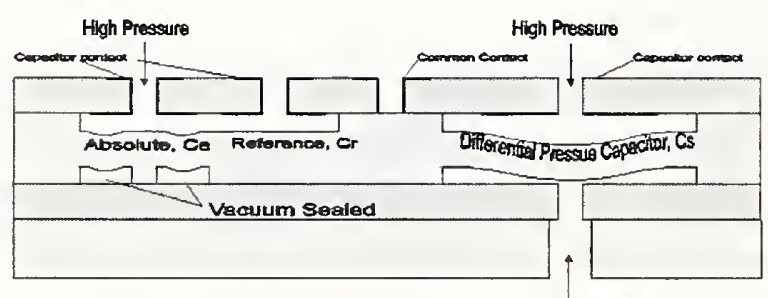

Low Pressure 


\section{Correction Model for a MV transmitter}

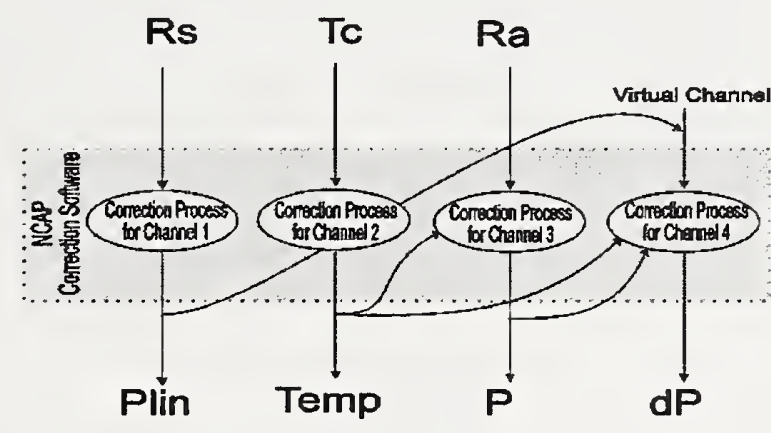

\section{Lessons Learned and Path forward}

- Not going to remake an established, commodity market

- IEEE 1451.2 is not a product!

- TII importance?

- Sensor mfg $\rightarrow$ OEM -No

- Sensor mfg $\rightarrow$ End user - Yes

- 1451 standards can provide the means to unleash existing sensor technology into new' markets and applications.

- Rapid growth of TCP/IP will create many new application that are hungry for sensor data. Many sensor teclinologies available to feed this need

- The goal of the 1451 standards is to bring these together 
"We have the trains, we have the places to go; the 1451 standards can provide the rails to make it happen!"

\section{Thank You}




\subsection{NI's Comments on IEEE 1451.2}
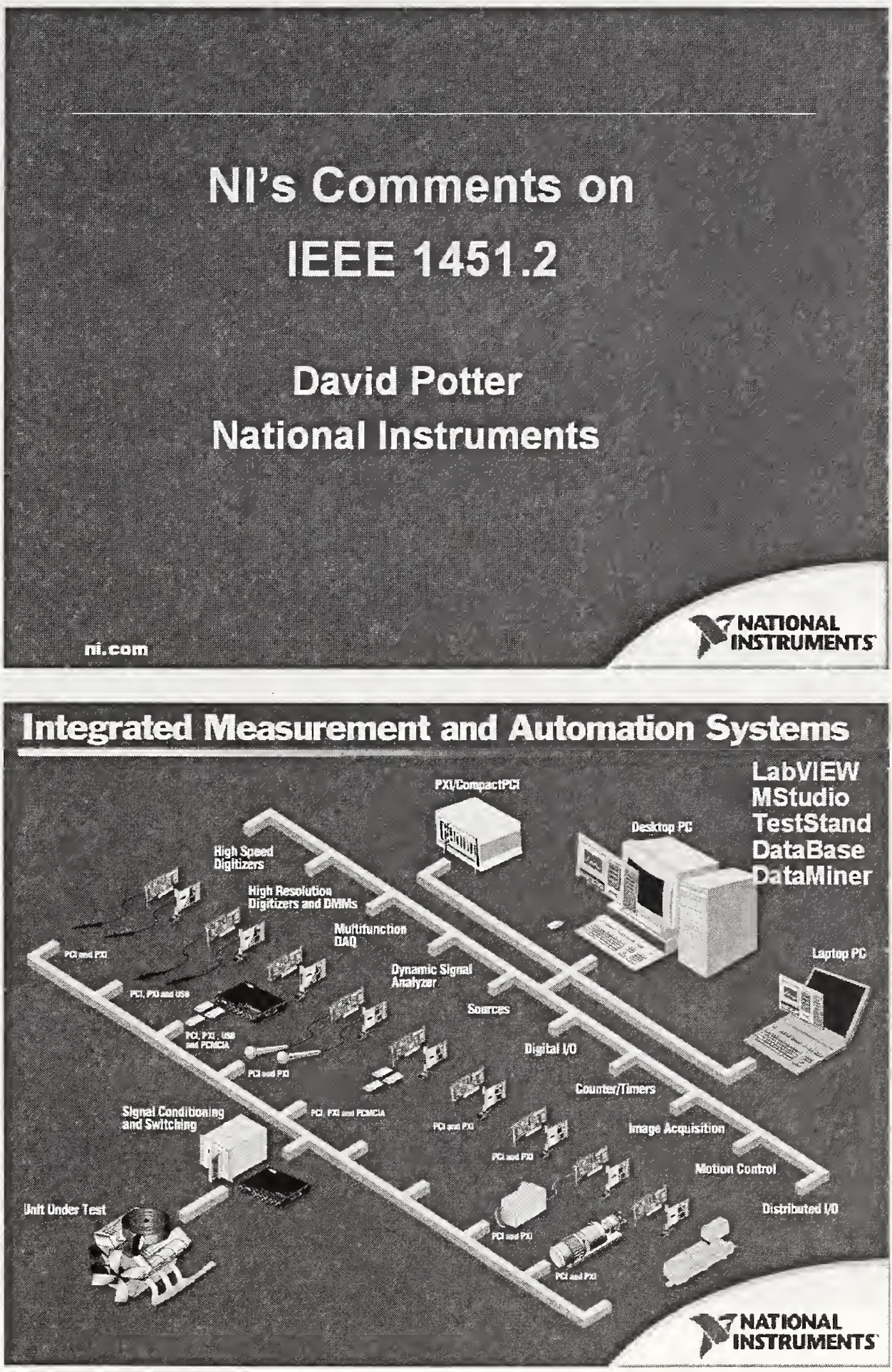


\section{NI and Smart Sensors: The Big Picture...}

- Smart sensors will be increasingly important in Measurement and Automation

- Main driver: easier connectivity and use of sensors

- 'Smart' sensors, smart modules, smart systems,...

- "What is smart" is less important than building integrated systems that deliver the end user benefits

- Nl as both software and hardware supplier

- As sortware supplier: support many networks, devices, instruments, as requested and required by customers

- As hardware supplier: highly selective of technology for interfaces

\section{As hardware supplier.. Possible Implementations?}

Plugin DAQ

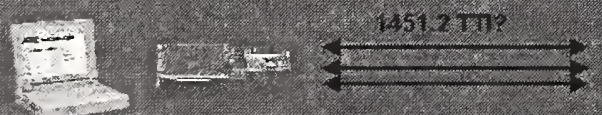

PXIDAR

* 11:[i!l

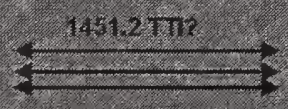

Networked 10 (DAO)

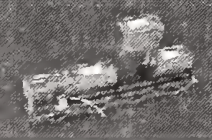




\section{Smart Sensor System Model}

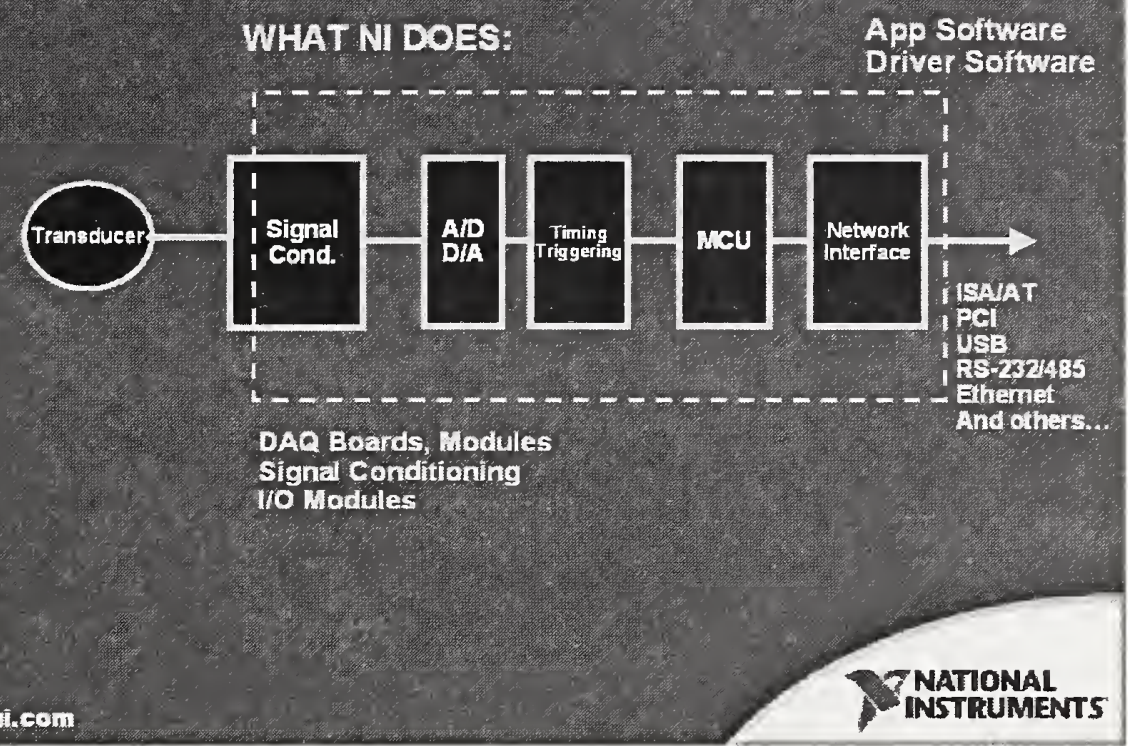

\section{Traditional Partitions}

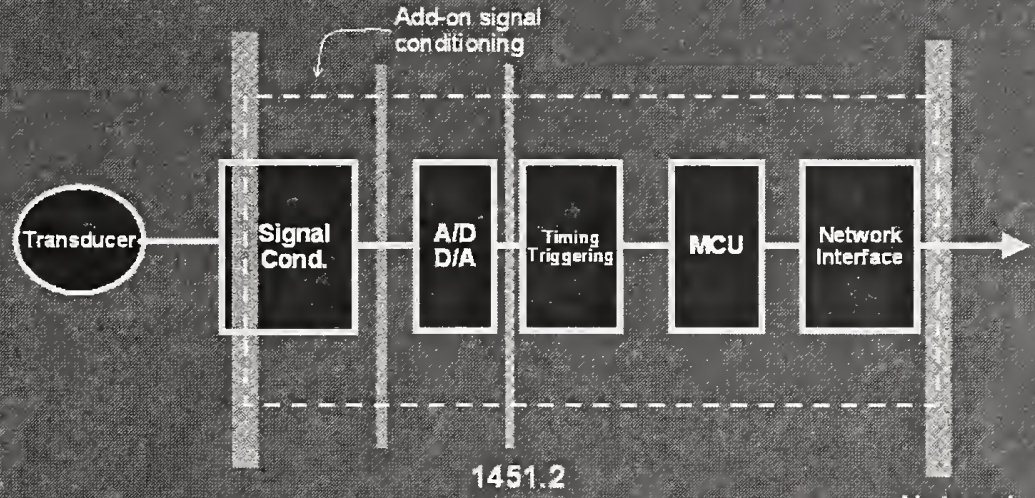

Analog

Network

(0-5V, 0-10V, mV, 4-20 mA) 


\section{NI's Thoughts/Reactions on dot2}

- Partition Location

- 1451.4 partition at analog is good int

- Seemed more appropriate as OEM (chip-level, module) level interface, rather than an end user interface

- Standardized TEDS can ease setup, configuration

- Particularly useful: calibration information

- Very useful if can somahow used across multiple electrical/physical implemantations

\section{"Generalized" TEDS?}

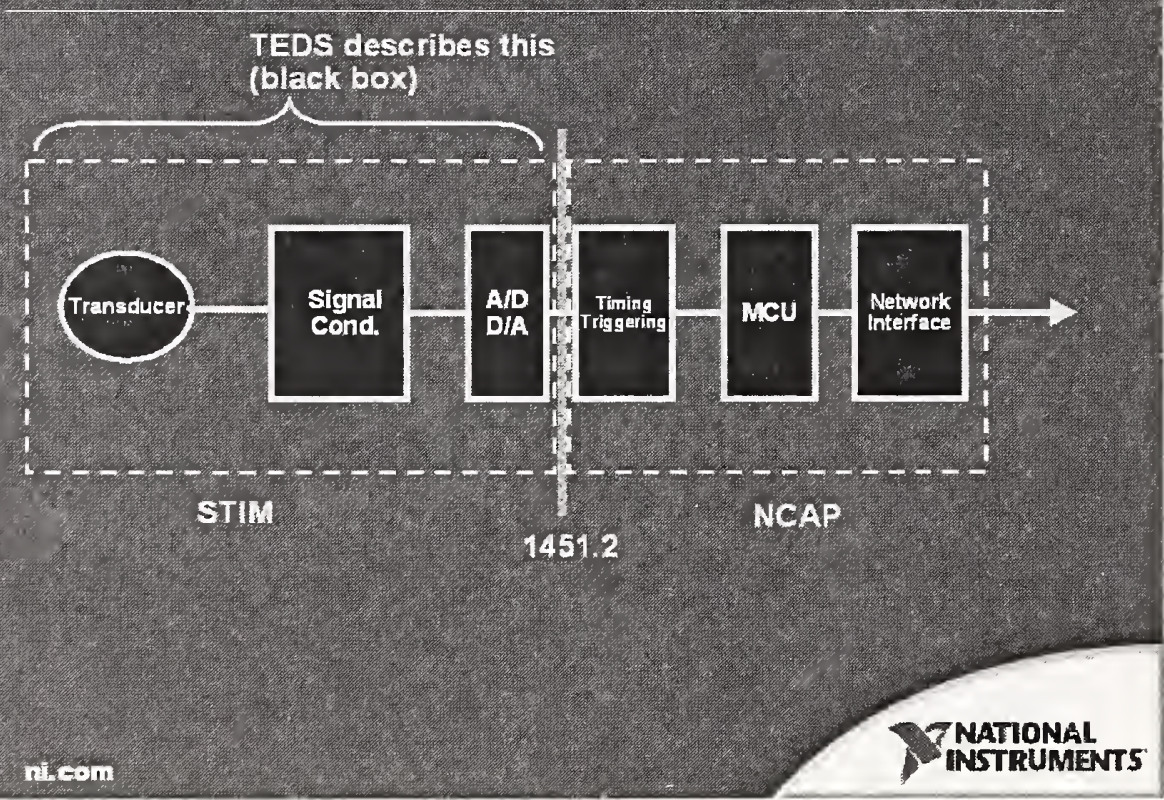




\section{"Ceneralized" TEDS?}

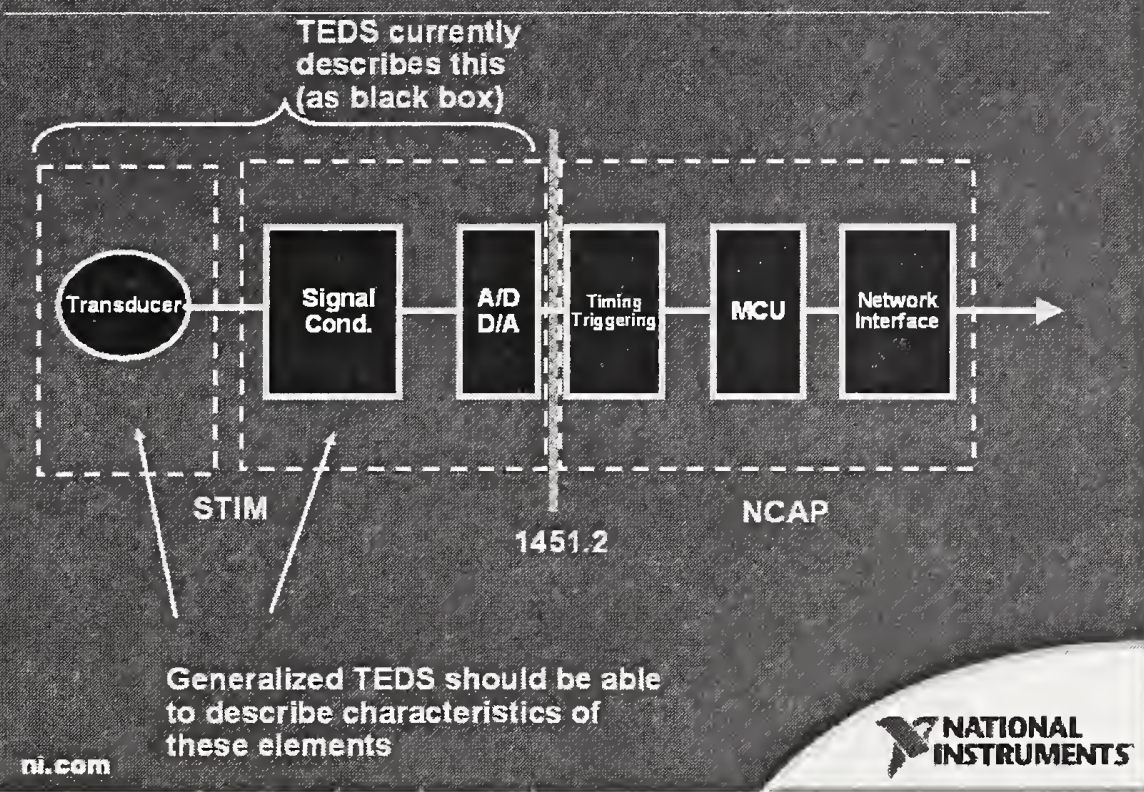

\section{Software}

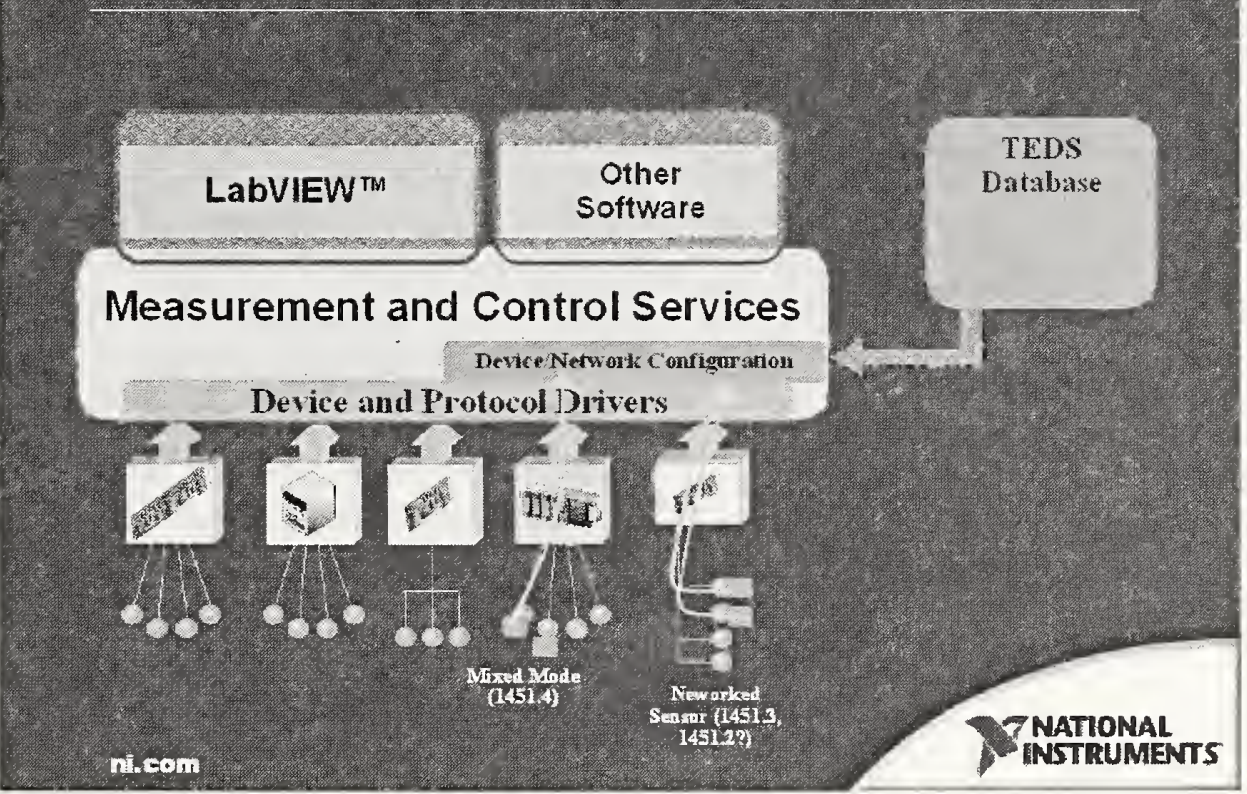




\section{NI's Thoughts/Reactions on $\operatorname{dot} 2$}

- Partition Location

- 1451.4 partition at analog is good if

- Seemed more appropriate as OEM (chip-level, module) level interface, rather than an end user interface

- Standardized TEDS can ease setup, configuration

- Particularly useful: calibration information

- Very useful if can somehow used across multiple electricallphysical implementations 


\subsection{Proposed Enhancements to the IEEE 1451.2 Standard for Smart Transducers}

\section{Proposed Enhancements to the IEEE 1451.2 Standard for Smart Transducers}

Robert N. Johnson, Telemonitor, Inc.

Stan Woods, Agilent Technologies, Inc.

October 4, 2001

\section{Most requested changes to IEEE 1451.2}

- Make it easier to understand and implement

- Make the hardware interface faster

- Use less wires

- Pick a standard connector

- Provide for electrical isolation

- Allow real-time reconfiguration

- Add frequency response to TEDS and correction engine

- Make NCAPs readily available and compatible with existing systems

- Don't add unnecessary expense to simple transducers

- Add security, timestamps, data logging, etc. 


\section{Proposed enhancements to IEEE 1451.2}

- Primary enhancements:

- Partition the TEDS

- Alternative physical layers

- Partition the standard

- Secondary enhancements:

- Enhance the TEDS

- Add functions

- Standalone function

- Corrections and additions

\section{Partition the TEDS}

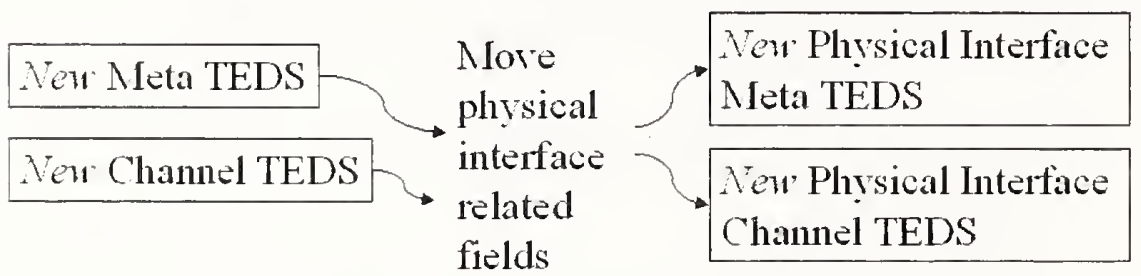

Meta-ID TEDS

Channel-ID TEDS 


\section{Proposed new IEEE 1451.2 TEDS blocks}

Machine readable

\begin{tabular}{|l|}
\hline $\begin{array}{l}\text { Meta-TEDS } \\
\text { (mandatory) }\end{array}$ \\
\hline Channel TEDS \\
(mandatory) \\
\hline Calibration TEDS \\
\hline $\begin{array}{l}\text { Physical layer } \\
\text { Meta-TEDS } \\
\text { Physical layer } \\
\text { Channel-TEDS }\end{array}$ \\
\hline
\end{tabular}

Octoher 4, 2001
Human readable

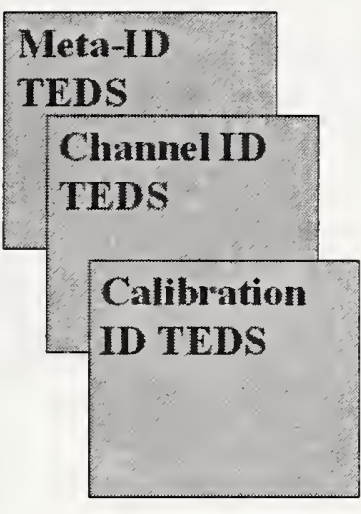

Application specific

End U'ser's'

Application specific TEDS

Future extensions

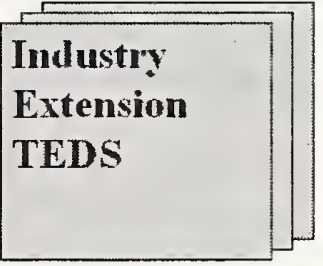

Sensors Expo, Philadelpha

Smart Transducer Interface Standard - IEEE 1451

\section{Alternate physical layers}

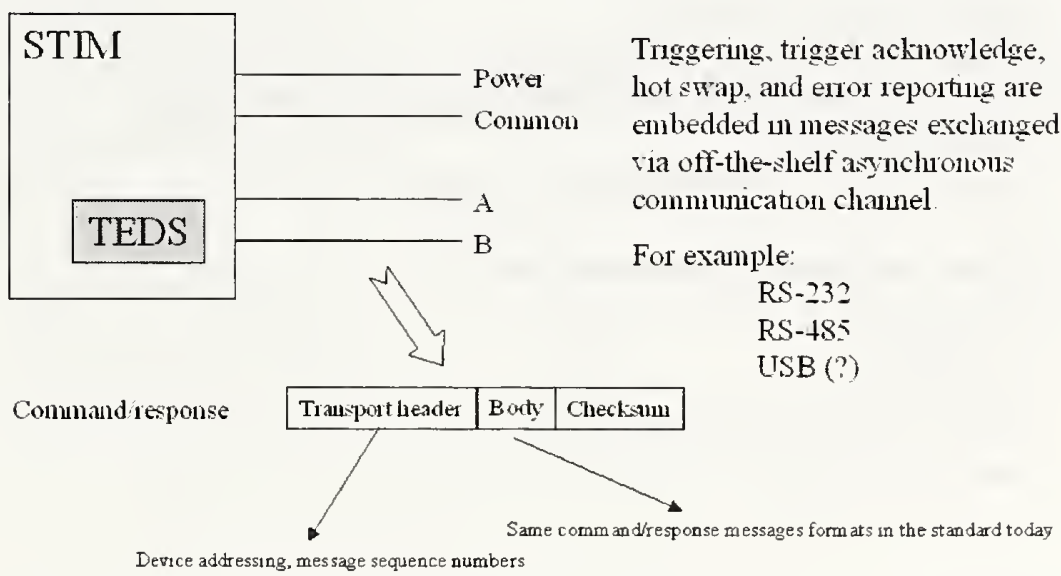




\section{Proposed IEEE 1451.2 serial interface}

1) Communication, triggering, error reporting 2) Power

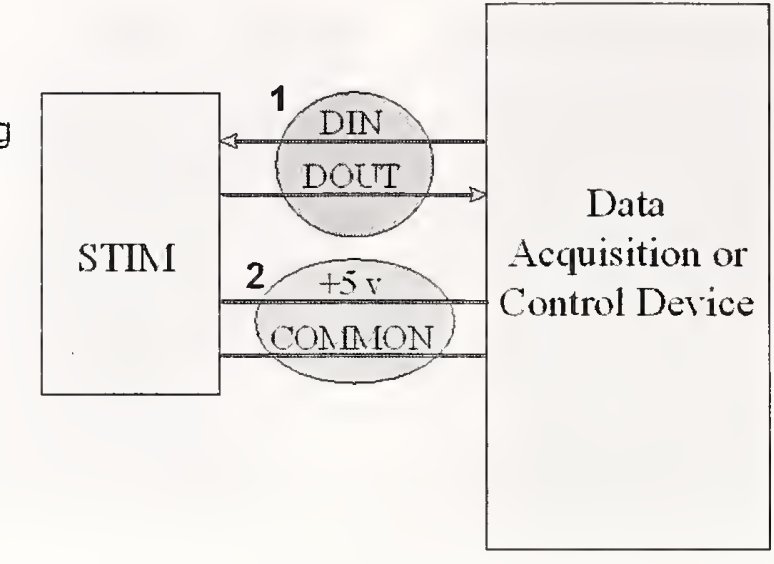

Signal names for 1) will depend on particular physical layer

Sensors Expo, Philadelpha

Smart Transducer Interface Standard - IEEE 1451

\section{Support for serial IEEE 1451.2}

Trends in UART support:

- present on most microprocessors

- chips have become smaller, less expensive, more robust

- multi-port UARTs

- supported in ADI Microconverter family

Where can we plug into serial ports?

- Instruments

- 1/O cards

- Computers

- VME, VXI, CPCI, PXI card cages

- Handhelds, PDAs 


\section{Partition the Standard}

- Organize the standard around the OSI information model

- Separate sections for major functions:

- TEDS

- Correction engine

- Physical layer

\section{Partition the standard (allow TEDS only)}

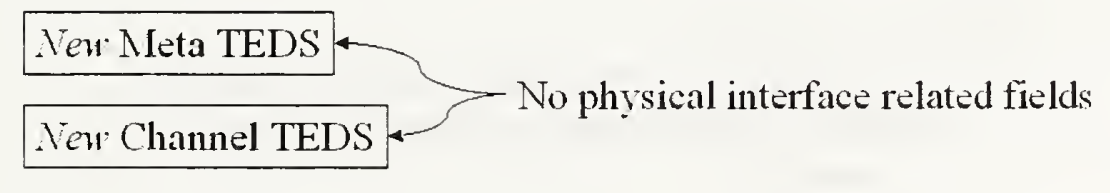

Meta-ID TEDS

Channel-II) TEDS $\vdots$ 


\section{Secondary enhancements}

- Enhance the TEDS

- Add features from IEEE P1451.3 and IEEE P1451.4

- Bandwidth

- Frequency response

- XML format

- Etc.

- Add functions

- Control function to tell NCAP to reload TEDS

- Support STIM reconfiguration:

- Gain, bandwidth, etc.

- Changes in channels due to hot-swap in local sub-net, including IEEE P1451.3 or IEEE P1451.4

\section{Secondary enhancements, con't.}

- Standalone function

- Support use with existing data and control systems

- Map IEEE 1451.2 functions to existing protocols:
- Modbus RTU
- Modbus/TCP
- Profibus
- HTTP URL-based
- XML
- Etc.

- Corrections and additions

- Miscellaneous comments received since publication

- Others identified during review and updating process 


\section{New connectivity enabled by enhancements}

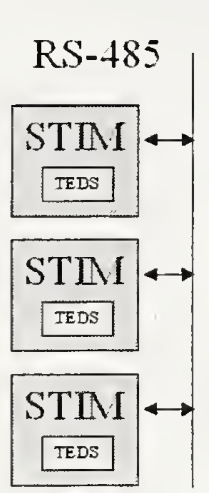

Octoher 4, 2001

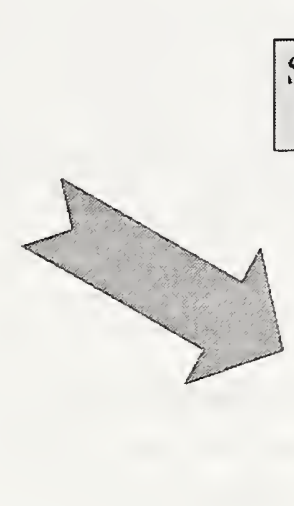

Seusor: Expo, Fhiladelphia
$\mathrm{RS}-232$

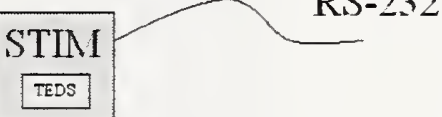

\section{Connectivity to existing} communication ports:

- Computers

- Programmable logic controllers

- PC plug-min cards

- Instruments

- NCAPs

\section{Applications for serial IEEE 1451.2}




\section{Multi-port serial device}

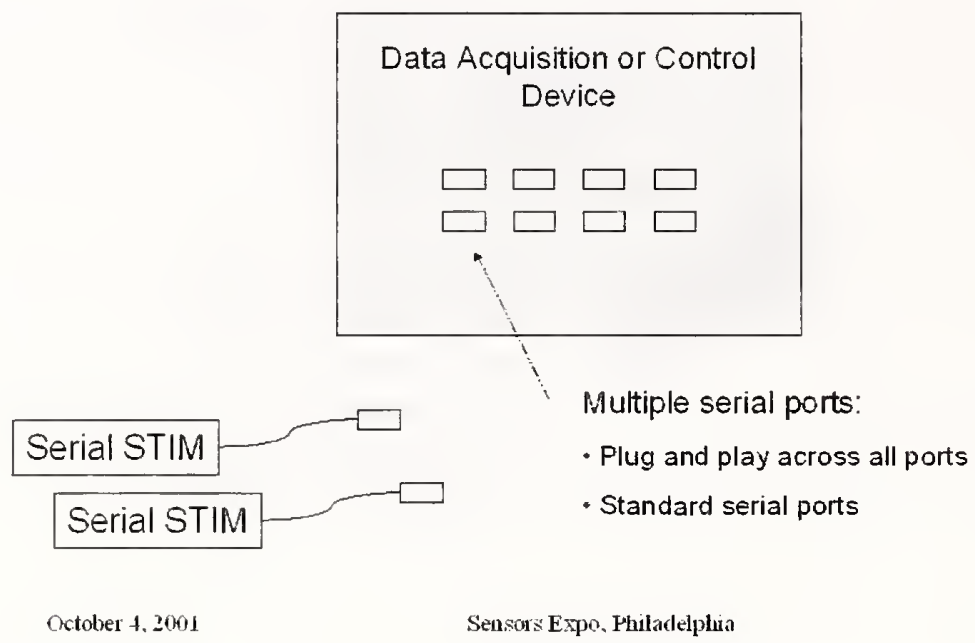

\section{Industrial card cage I/O card}

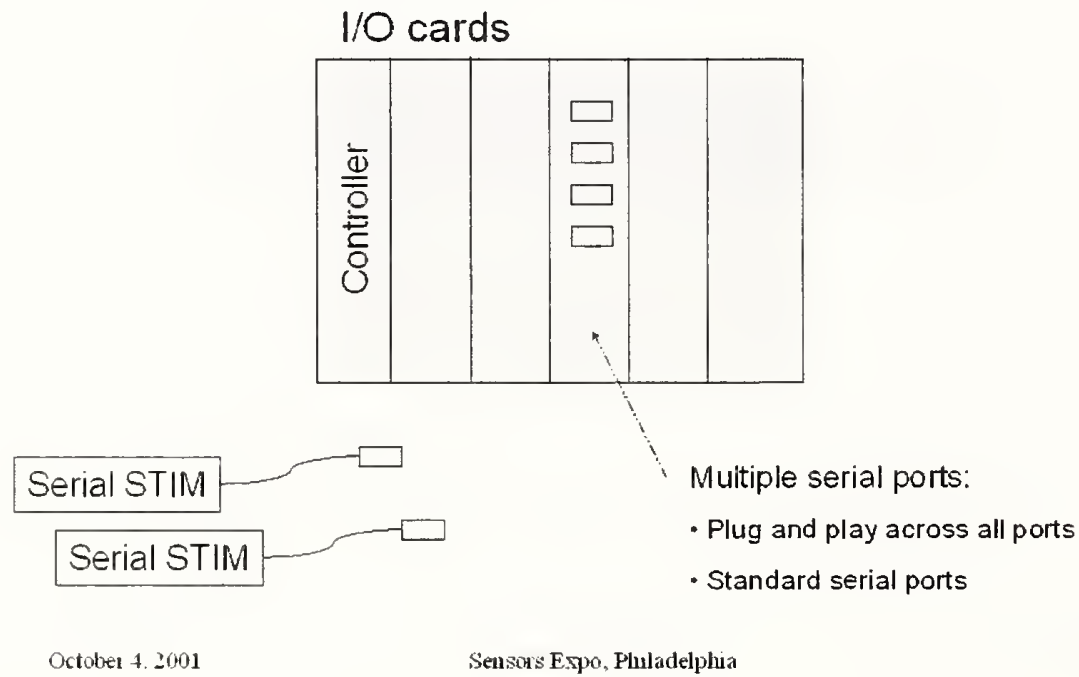

16 


\section{Computers and PDAs}

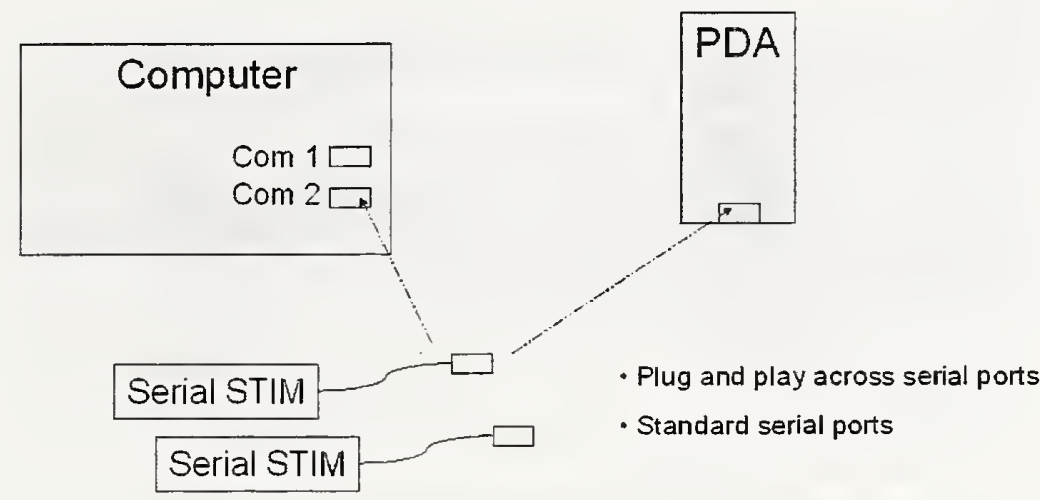

\section{Most requested changes to IEEE 1451.2}

- Make it easier to understand and implement

- Make the hardware interface faster

- Use less wires

- Pick a standard connector

- Provide for electrical isolation

- Allow real-time reconfiguration

- Add frequency response to TEDS and correction engine

- Make NCAPs readily available and compatible with existing systems

- Don't add unnecessary expense to simple transducers

- Add security, timestamps, data logging, etc. 


\section{Conclusions}

- IEEE 1451.2 established several valuable basic principles of smart transducers

- The most important of these is the TEDS

- We need to keep the best parts of the original standard while addressing the current needs of the marketplace

- The proposed enhancements will address the major requested changes and will result in:

- More flexibility

- Lower cost

- Improved connectivity

- We need user comments and feedback on the proposed enhancements

\section{Questions/Comments?}




\section{Proposed serial version of IEEE 1451.2}

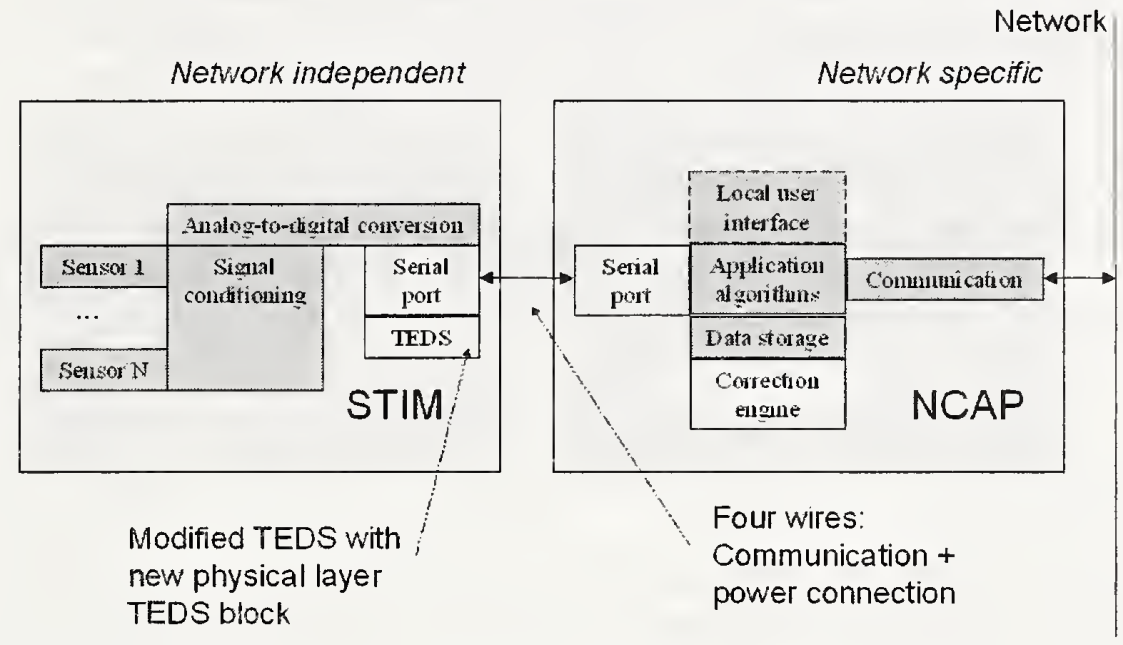

\section{Where to put the STIM interface electronics?}

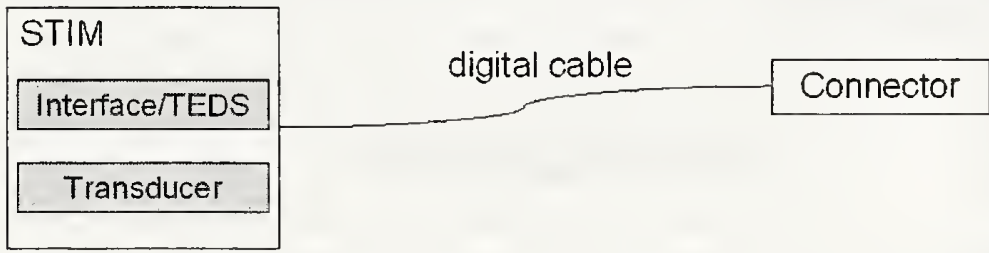

\begin{tabular}{|l|l|}
\hline Transducer & Connector \\
\hline
\end{tabular}




\section{Presentations From "Second Workshop on Wireless Sensing"}

\subsection{An IEEE 1451.1 Summary}

\section{An IEEE 1451.1 Summary}

Rick Schneeman, Computer Scientist

rschneeman@nist.gov

US Department of Commerce

National Institute of Standards and Technology (NIST)

Gaithersburg, Maryland 20899 USA

\section{Introduction}

- Who we are: NIST mission is to help increase US industry competitiveness through advanced research, standards, and technology collaboration

- Member of the Sensor Development and Application Group (SDAG) within the Manufacturing Engineering Laboratory (MEL) at NIST

- Member of the Working Group on the IEEE Standard for a Smart Transducer Interface for Sensors and Actuators - Network Capable Application Processor (NCAP) Information Model, or IEEE 1451.1 ("dot1") 


\section{IEEE 1451 Overview/Goals}

- Provide standardized communication interfaces for smart transducers, both sensors and actuators. In the form of a standard hardware and software definition/specification.

- Simplify the connectivity and maintenance of transducers to device networks through such mechanisms as common Transducer Electronic Data Sheet (TEDS) and standardized Application Programming Interfaces (API)

- Allow plug-and-play with 1451 compatible transducers among different devices using multiple control networks

- Give sensor manufacturers, system integrators, and endusers the ability to support multiple networks and transducer families in a cost effective way

An IEEE 1451.1 Summary

\section{Part 1: IEEE 1451.1 Overview/Goals}

- "The specifications provide a comprehensive data model for the factory floor. and a simple application framework to build interoperable distributed applications..." Dr. Jay Warrior, Agilent Technologies, Chair IEEE 1451.1 WG

- In general, IEEE 1451.1 accomplishes this by providing:

- Transducer application portability (software reuse)

- Plug-and-play software capabilities (components)

- Network independence (network abstraction layer)

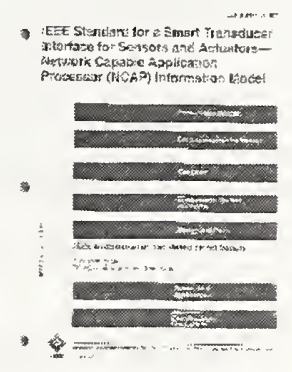

- The standard specifies these capabilities by defining software interfaces for:

- Application functions in the NCAP that interact with the network that are independent of any network

- Application functions in the NCAP that interact with the transducers that are independent of any specific transducer

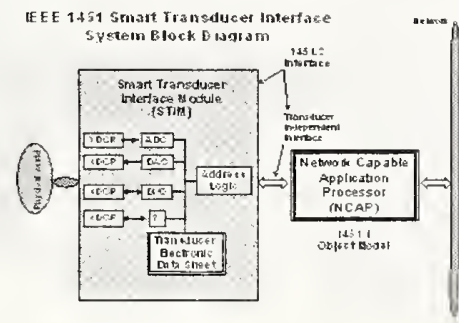

An IEe driver interface 


\section{IEEE 1451.1 Overview/Goals (Cont.)}

- IEEE 1451.1 software architecture is defined using three different models or views of the transducer device environment:

- An Object Model, defines transducer device specific abstract objects - or, classes with attributes, methods, and state behavior

- A Data Model, defines information encoding rules for transmitting information across both local and remote object interfaces

- A Network Communication Model, supports a client/server and publish/subscribe paradigm for communicating information between NCAPs

\section{Conceptual View of an IEEE 1451.1 NCAP}

- Uses a "backplane" or "card cage" concept

- NCAP centralizes and "glues" all the system and communications facilities together

- Network communication viewed through the NCAP as ports

- Function block application code is "plugged" in as needed

- Transducer blocks map the physical transducer to the NCAP 


\section{IEEE 1451.1 Communication Model}

- Provides two styles of inter-NCAP communication

- Client/Server: A tightly coupled, point-to-point model for one-to-one communication scenarios - typically used for configuration, attribute accessors, and operation invocations

- Publish/Subscribe: A loosely coupled, model for many-to-many and one-to-many communication scenarios - typically used for broadcasting or multicasting measurement data and configuration management (i.e., node or NCAP discovery) information

\section{Implementing IEEE 1451.1}

- An IEEE 1451.1 C++ Reference Implementation provides a concrete representation of the abstract Smart Transducer Information Model (IEEE Std 1451.1-1999, Dated 18 April 2000). The NIST implementation is called "1451.1 Lite", as it is a subset of the complete specification.

- A subset of the IEEE 1451.1 implementation has also been developed in Java to provide an architecture neutral NCAP configuration tool.

- The C++ implementation uses the open-source Adaptive Communication Environment (ACE) from the Washington University at St. Louis. 


\section{IEEE 1451.1 Benefits}

- Using P1451.1 provides:

- an extensible object-oriented model for smart transducer application development and deployment

- application portability achieved through agreed upon application programming interfaces (API)

- network neutral interface allows the same application to be plug-and-play across multiple network technologies

- leverages existing networking technology, does not reimplement any control network software or protocols

- a common software interface to transducer hardware i/o

\section{Looking at an IEEE 1451.1 Application}

- A minimal IEEE 1451.1 application consist of a few classes:

- An NCAP Block (consolidates system and communication housekeeping)

- A Transducer Block (provides the software connection to the transducer device)

- A Function Block (provides the transducer application algorithm (i.e., obtain and multicast temperature data every second)

- Parameters (contains the network accessible variables that hold and update the data)

- Ports (network communication objects for publishing and subscribing to information or interacting with other NCAPs using client/server 


\section{Executing an IEEE 1451.1 Application}

- An embedded Temperature NCAP Application is running from a remote location on the NIST Intranet

- As part of the system configuration. a NIST developed Java tool on a Notebook issues a discovery multicast, finds the NCAP. and starts the remote NCAP's Function Block

- The remote NCAP Function Block responds by publishing temperature data every second as the Java tool records the information

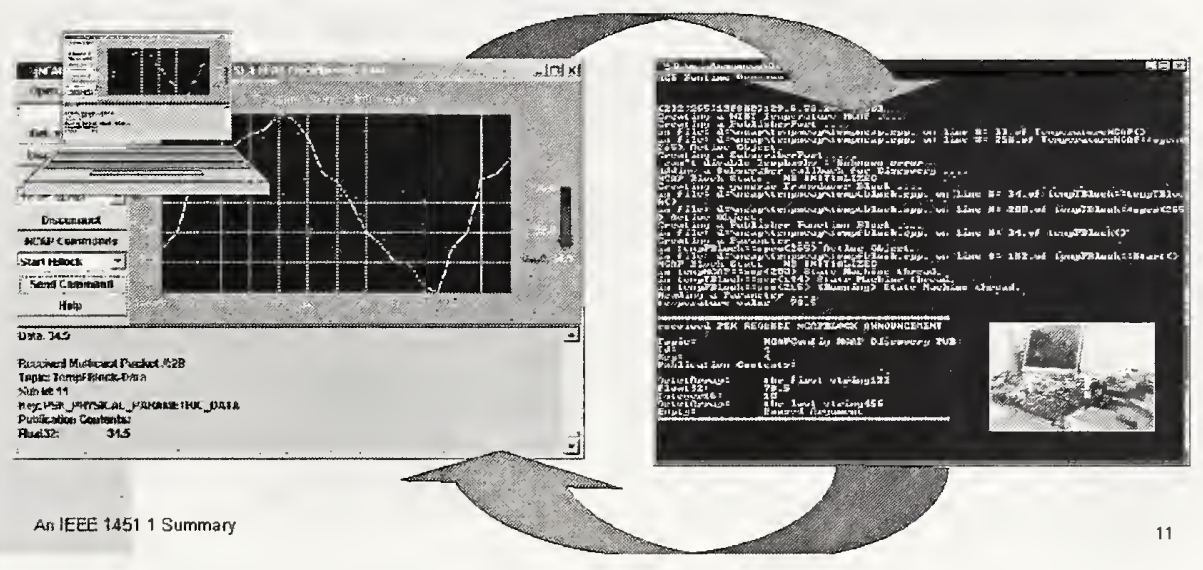

\section{How NIST uses IEEE 1451.1 in a Wireless Environment}

- The NIST C++ IEEE 1451.1 reference implementation uses TCP/IP as its underlying control network.

- From TCP/IP, IP multicast and TCP unicast features are used to implement publish/subscribe and client/server, respectively

- ACE is used to abstract the networking code from the application; therefore it is highly adaptive to various protocols

- Wired 802.3 Ethernet has been used primarily for testing. No changes were needed in ACE to support this protocol.

- Wireless 802.11b (11Mbps) Ethernet has also been used for testing. Again, no changes were made to ACE as the TCP/IP protocol is compatible with both 802.3 and 802.11 b physical mediums. 


\section{How NIST uses IEEE 1451.1 in a Wireless Environment}

- Testing scenarios included using a wired subnet connected to a wireless extension of the subnet

- Wireless extension uses an Agere (formerly Lucent) Orinoco AP-1000 dual card "access point"

- Range extender antennas are also connected to the access point and each PC-CARD

- Each node on the wireless side executes an IEEE 1451.1 NCAP application

- Java Configuration tool executes beyond the wireless net on the wired subnet
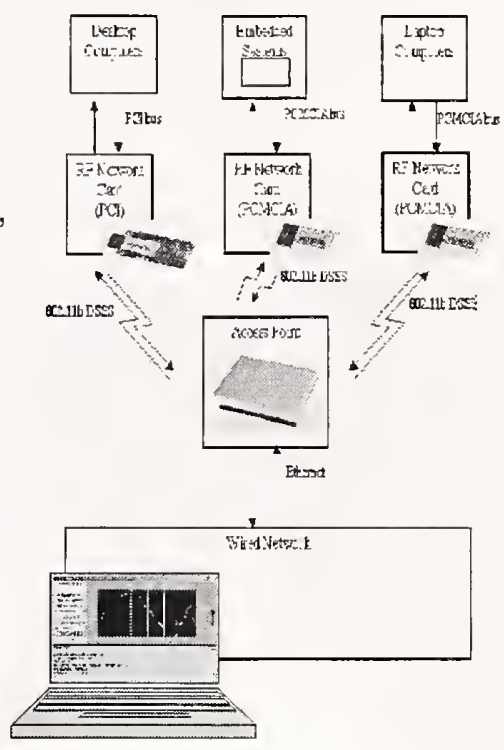

An IEEE 1451. 1 Summany

\section{Summary}

- IEEE 1451.1 is a large and comprehensive standard that addresses the needs of the smart transducer industry for providing portability and network independent access.

- NIST has begun implementing a good deal of the standard with emphasis on getting the software communication and infrastructure in place in order to start using the code.

- Choosing and implementing the standard with a solid object-oriented framework such as ACE provides a robust environment for real-time network communication.

- Migrating the implementation to other middleware such as CORBA for heavier weight uses will be reasonable to do

- Several projects at NIST will use the implementation for supporting manufacturing related activities 


\section{Summary (cont)}

- Continued testing in the wireless space is required to gauge the effectiveness of the implementation.

- Bluetooth trials are forthcoming; however, the lack of multicast support will severely impact the applications - continued research here is a must

- Other lightweight middleware packages are going to be isolated $-x \mathrm{ml}$ and soap, etc; however, these protocols do not support asynchronous messaging or publish subscribe in efficient ways

- Slimmer implementations of the IEEE 1451.1 will need to be experimented with for use with the smaller micro platforms.

\section{For more information....}

- ACE can be found at:

www. cs. wristI. edu/ schmidt/ACE. htmI

- 1451.1-1999 IEEE Standard for a Smart Transducer Interface for Sensors and Actuators - Network Capable Application Processor Information Model 2000: ISBN 0-7381-1768-4

- The NIST IEEE 1451 Web Site provides information about 1451, publications, and demonstrations at: ieee1451.nist.gov 


\subsection{Bluetooth for 1451}

\section{Bluetooth for 1451}

\section{Sensor Area Networks (SANs)}

\section{Thurston Brooks}

IEEE P1451 4 \& P1453.3 Member

Bluetooch LA SG Vice Char

with contnbutions by

Ericsson Mobile Communications $A B$

\section{Wireless Monitoring}

- Will reduce monitoring installation cost

- \$_0ft typical, as much as $\$ \geq 000 / \mathrm{ft}$ some apps (e g., Nuclear)

- Cablung is $30-45 \%$ of TOC

- Spread spectrum technology is now arailable for low cost

- Mobile connections

- Real-time dynamic range is expensive

- Power

$-\operatorname{Cost} \$$

- Immediate alert of alarm conditions and follow-up data dumps are also feasible 


\section{Machinery Monitoring Implementation with NCAP}

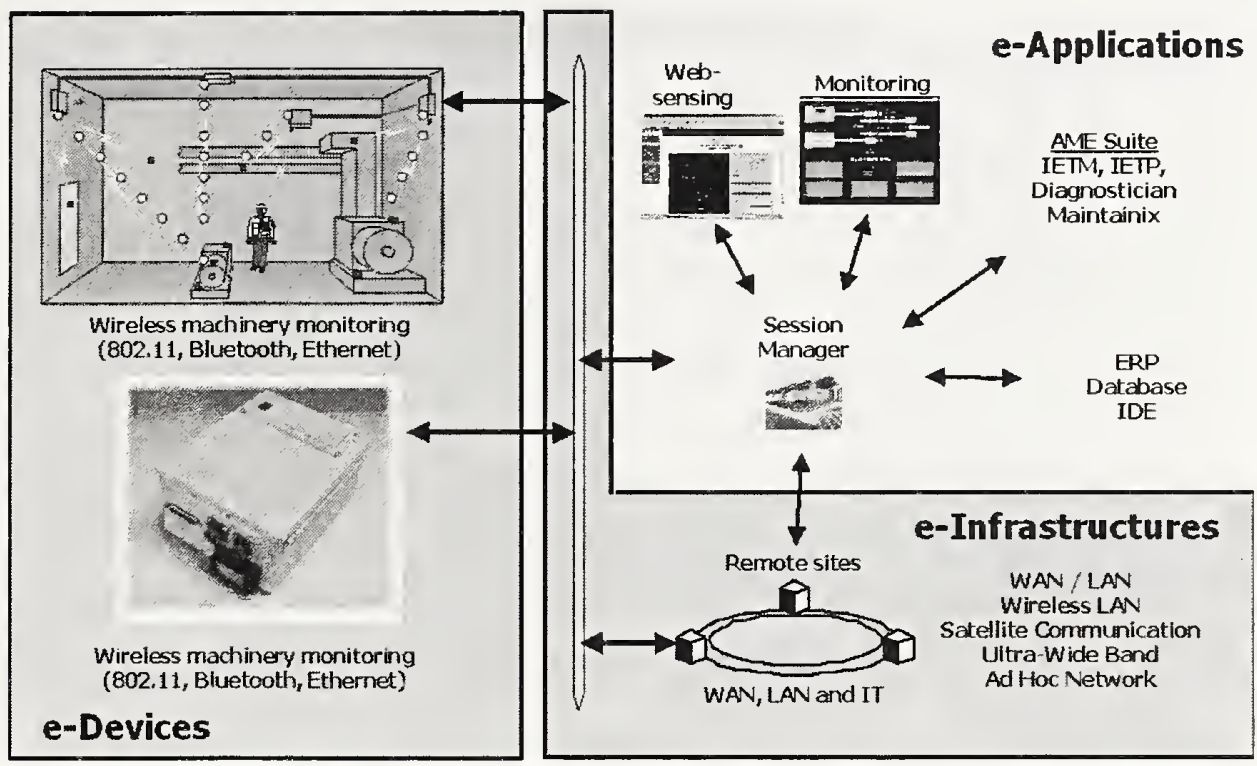

Sensors Expo 2001 - Bluetooth SANs

3 e Technologies International. Inc.

\section{Wireless Manufacturing Solutions}

- Majority of wireless products in marketplace are proprietary spread spectrum and narrow band solutions in the ISM bands (400M $\mathrm{Mz}, 900 \mathrm{MHz} .2 .4 \mathrm{GHz})$

- Today $80^{\circ} \%$ of customers $\rightarrow$ SS (*amer Group)

- Typically constrain user to buy from a particular vendor

- Interoperability, low-cost, and broad user base (i.e., market demand) have been stimulated by Standards

- IEEE 80211 (2.4GHz (a1-2Mbps)

LAN

- Bluetonth (2.4GHz@. $75 \mathrm{Mbps}$ )

PAN

- Potential Interference between S02.11 and Bluetooth 


\section{Bluctooth Features}

- Low-Cost

- Higldy Integrated ASICs

- Low-Power

- Small Size

- Frequencies

- covers all of the $2.4 \mathrm{GHz}$ ISM band

- Tine Division Duplex

- Stand-by Modes

- Palk

- Hold

- Suift

- Standby

\section{Bluctooth Link Budget for Max Power TX}

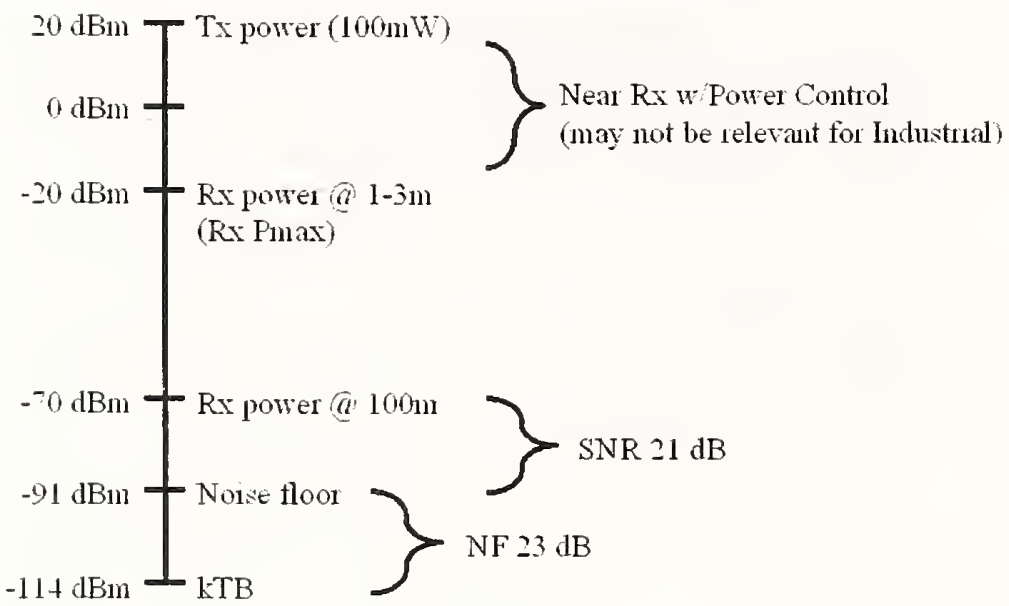




\section{Operational States}

- Standby

- Inquiry-Paging

- Connections

- Active

- Power Conserving

- Hold (delta t)

- Sniff (Poling)

- Park (Lsiten but do not disturb master)

- Synchronous Connection-Oriented (SCO) Link (primarily used for voice - could be used for deterministic link)

- Asynchronous Connection-Less (ACL) Link (packet data)

\section{Operational States}

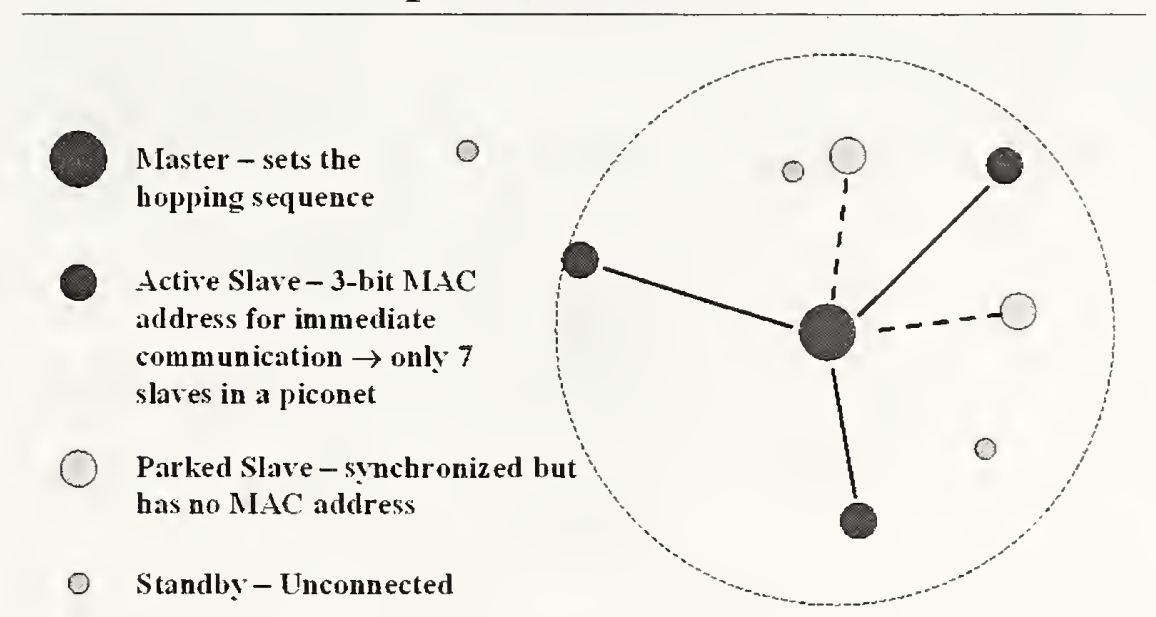




\section{Making a Connection}
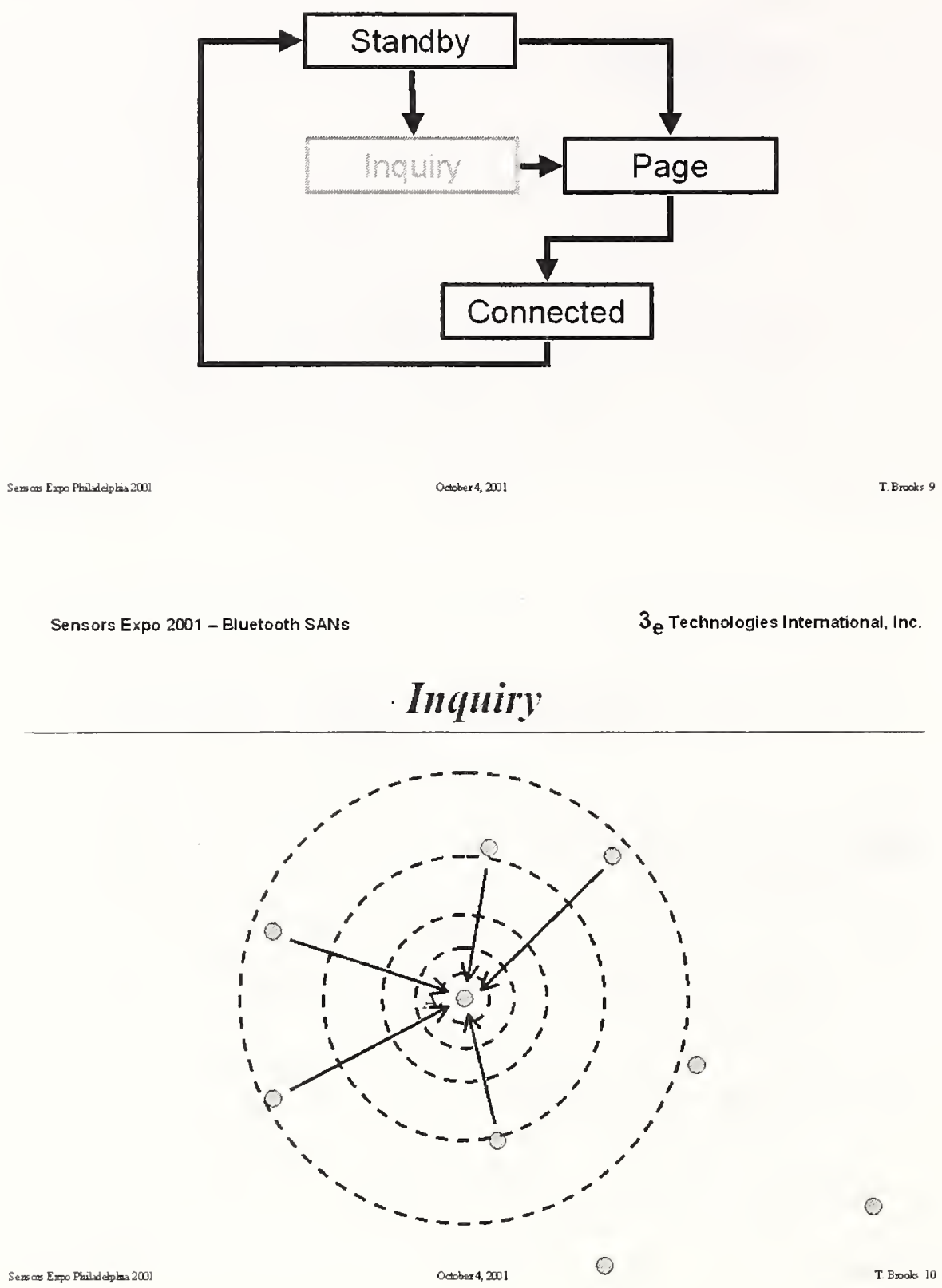
Sensors Expo 2001 - Bluetooth SANs $\quad 3 \mathrm{e}^{\text {Technologies International, Inc. }}$

\section{Making a Connection}

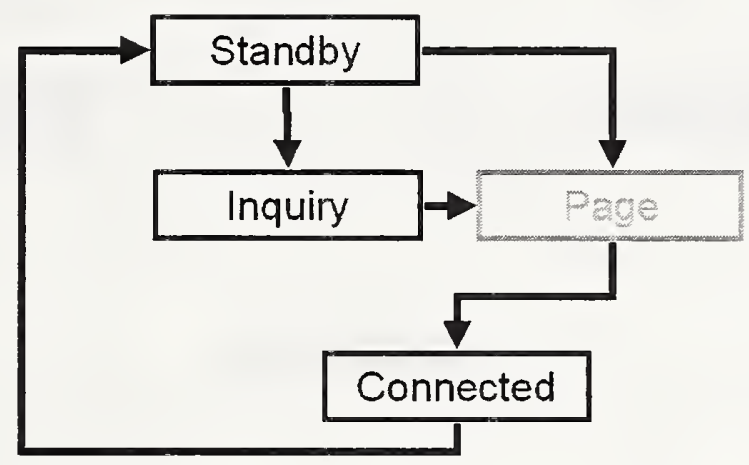

\section{Creating a Piconet}

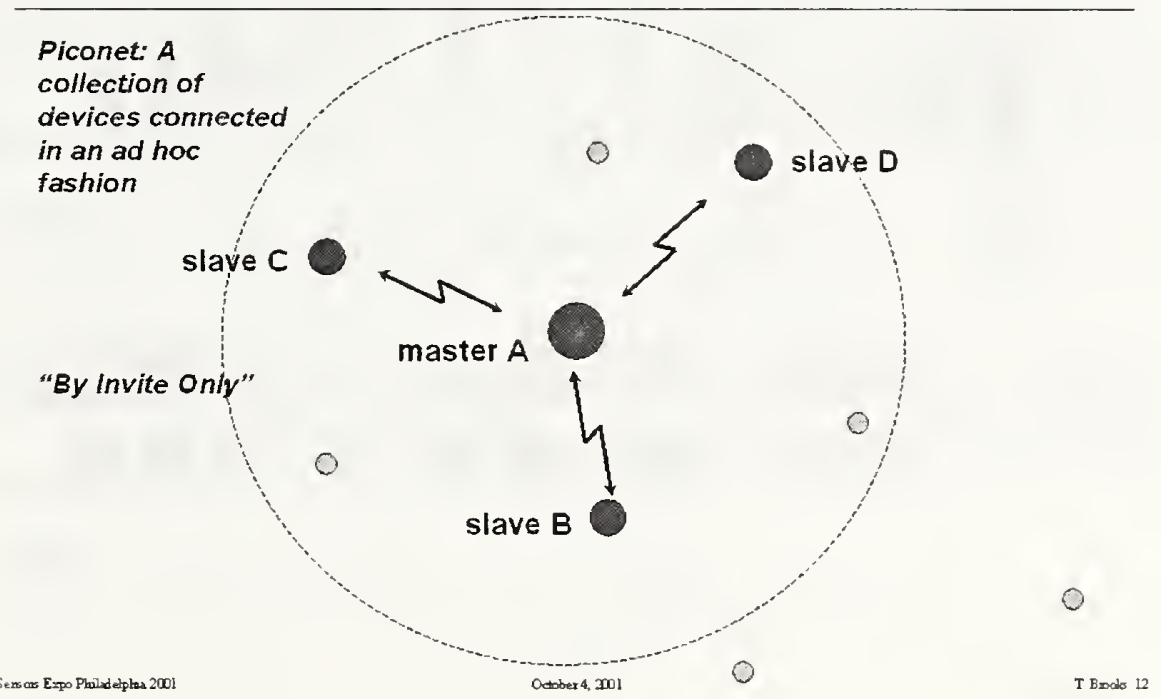


FH with TDD Channel
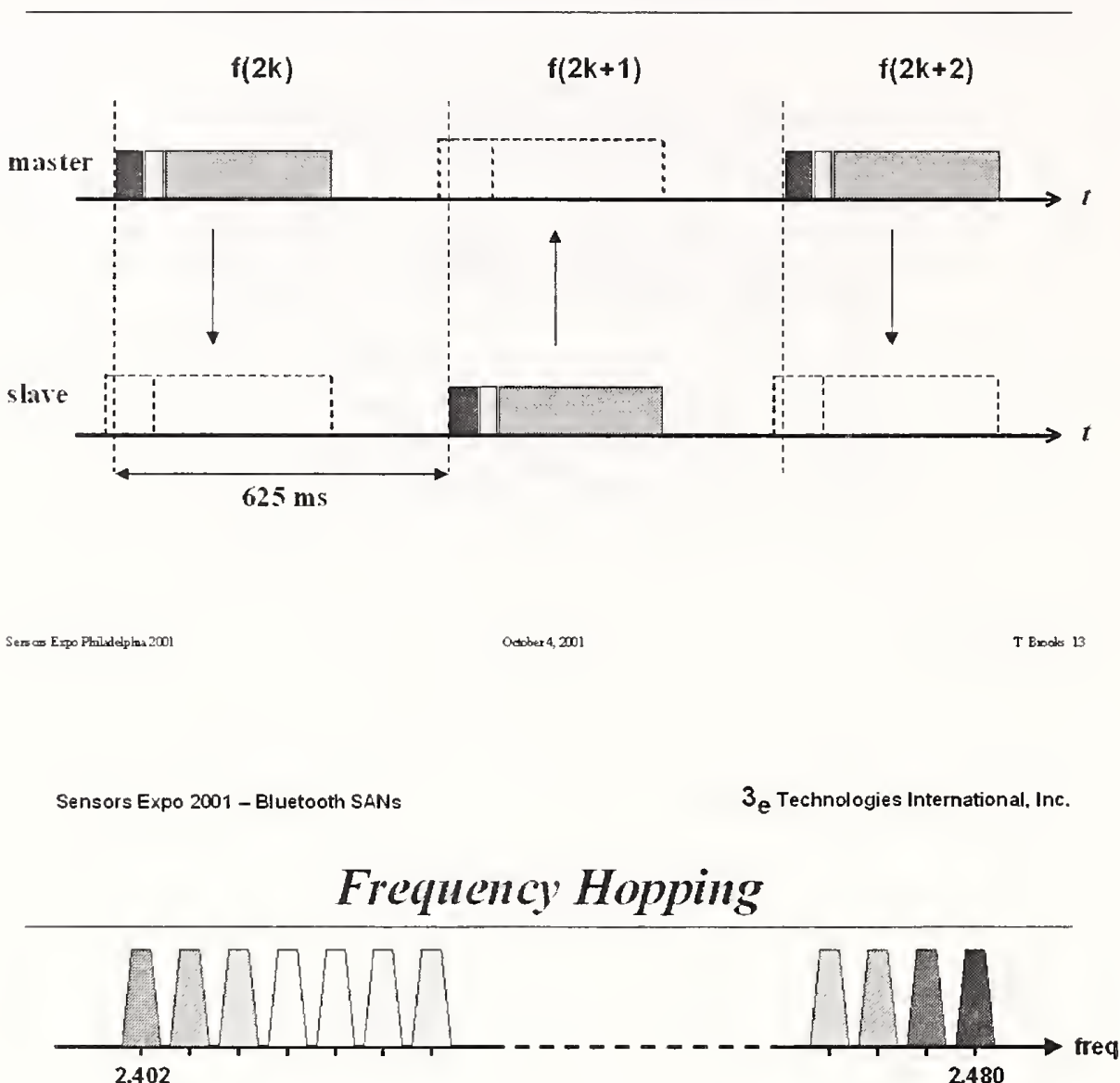

master

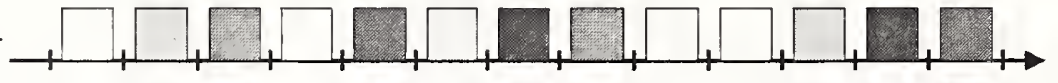

slave

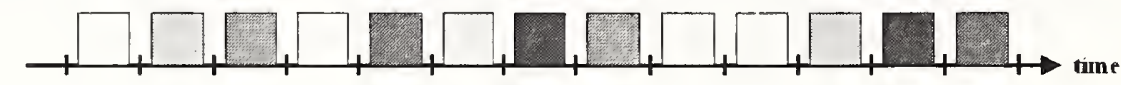




\section{Synchronisation of Physical Channel}

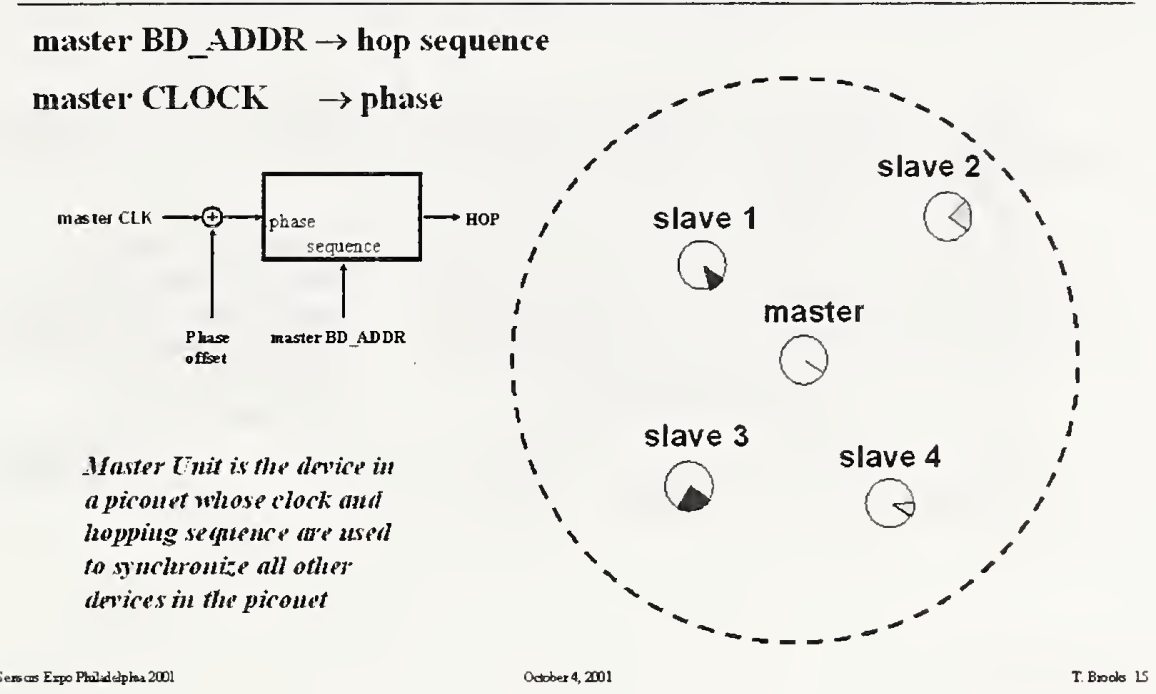

Sensors Expo 2001 - Bluetooth SANs

$3 \mathrm{e}^{\text {Technologies International, Inc. }}$

\section{Mixed Link Example}

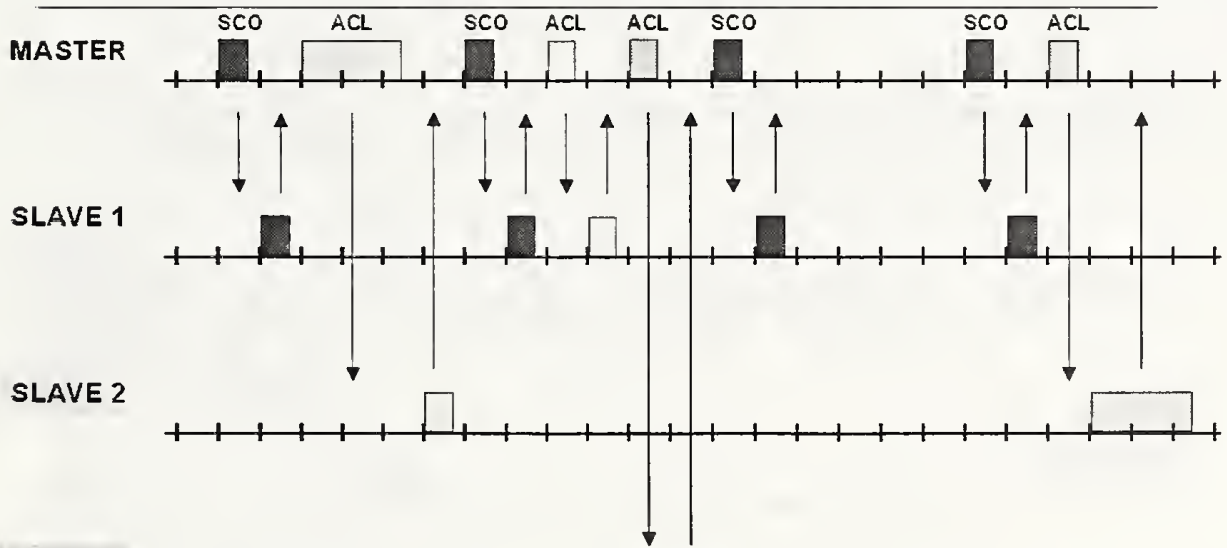

SLAVE 3

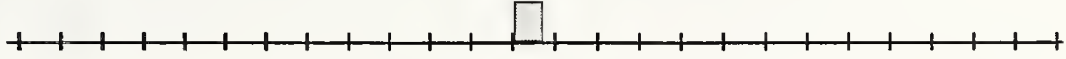




\section{Data Rates (kb/s)}

\begin{tabular}{|c|c|c|c|}
\hline type & symmetric & asym & etric \\
\hline DM1 & 108.8 & 108.8 & 108.8 \\
\hline DH1 & 172.8 & 172.8 & 172.8 \\
\hline DM3 & 258.1 & 387.2 & 54.4 \\
\hline $\mathrm{DH} 3$ & 390.4 & 585.6 & 86.4 \\
\hline DM5 & 286.7 & 477.8 & 36.3 \\
\hline DH5 & 433.9 & 723.2 & 57.6 \\
\hline
\end{tabular}

Master unit controls the link bandwidth, decides how much is given to each slave, and sets the symmetry of the link

\section{Automatic Repeat Request (ARO) Scheme}

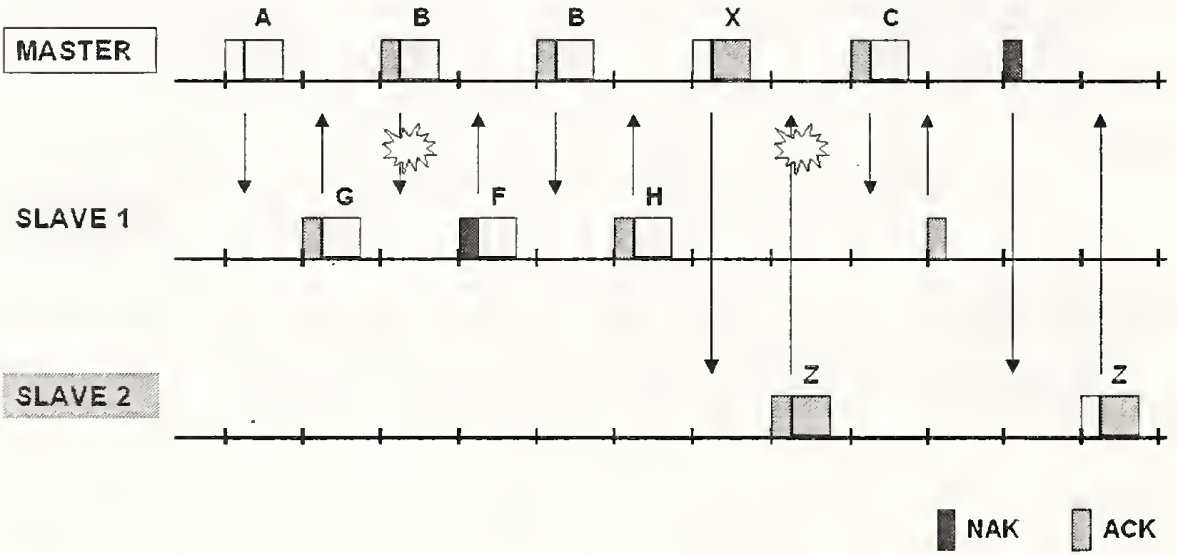




\section{Sniff Mode}
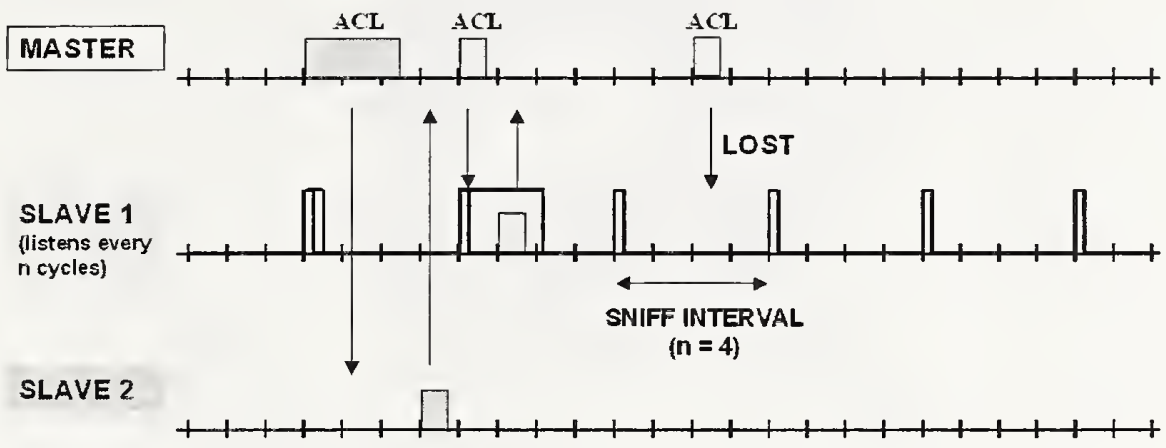

\section{Park Mode}

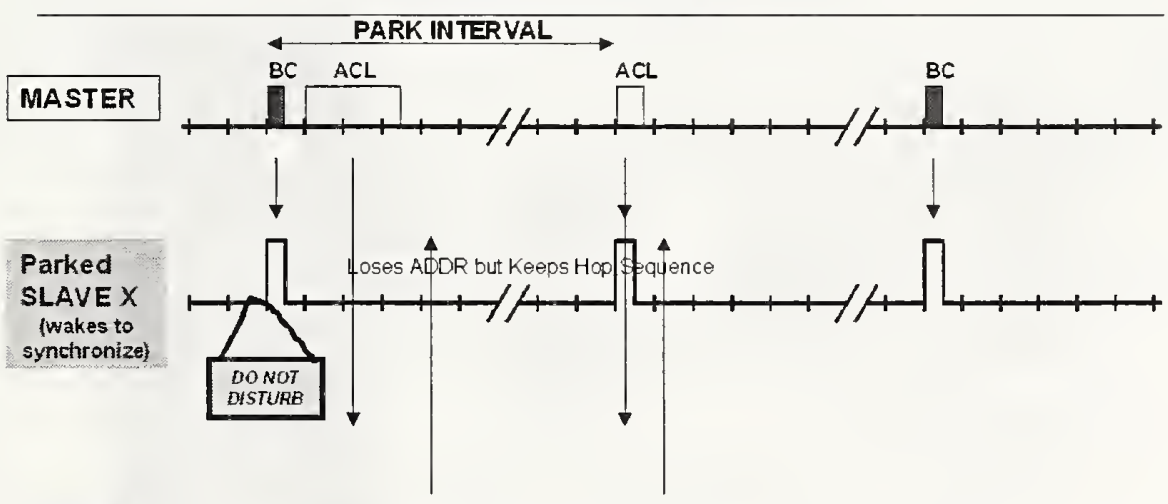

SLAVE 2

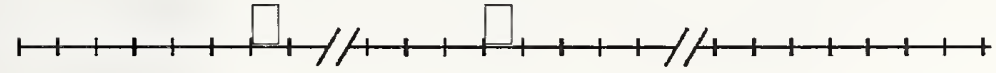




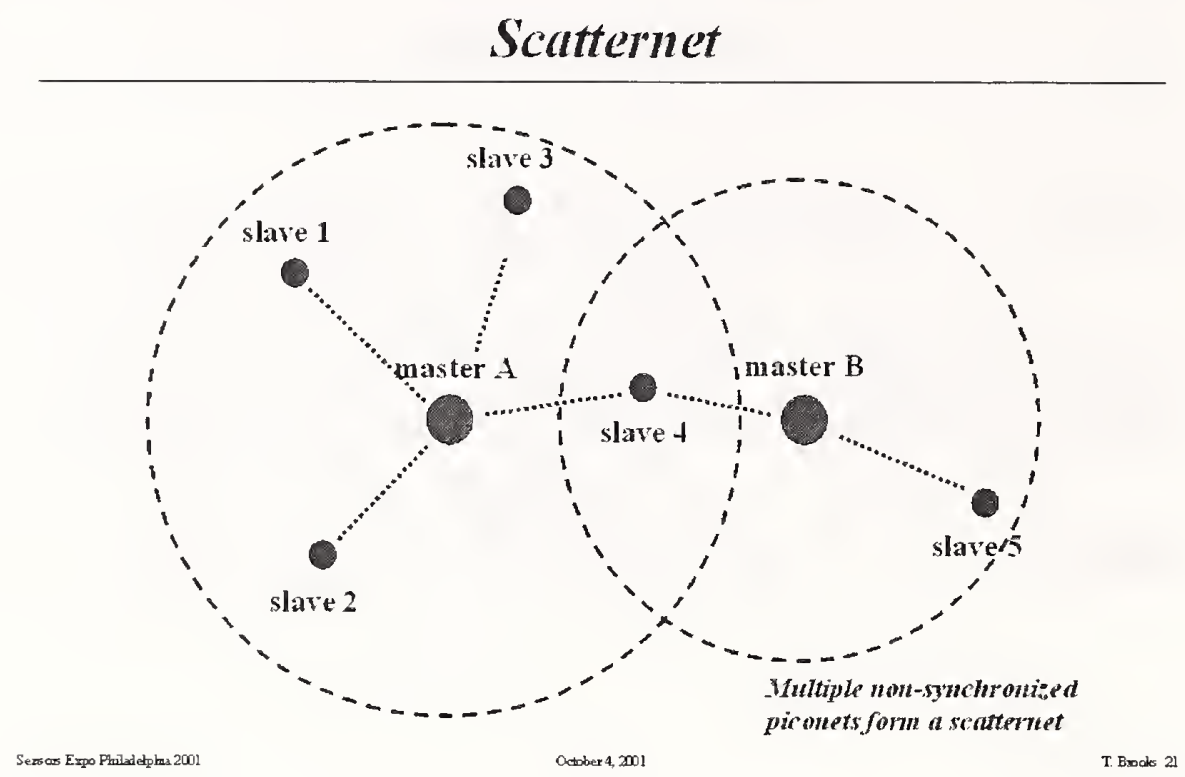

\section{Wireless NCAP Unit}

- Watertight

- Small size ( 8 W x $10 \mathrm{H}$ x 5D)

- IEEE 802.11b or Bluetooth with Antemua diversity

- Comectorzed

- Labtien HMI

- Elght 1451 smat sensor input: usug modular plug-u design

o Vibration

a Temperature

o Pressure

o Flow

- Etc

- Future Revisions

a V,oo Modem

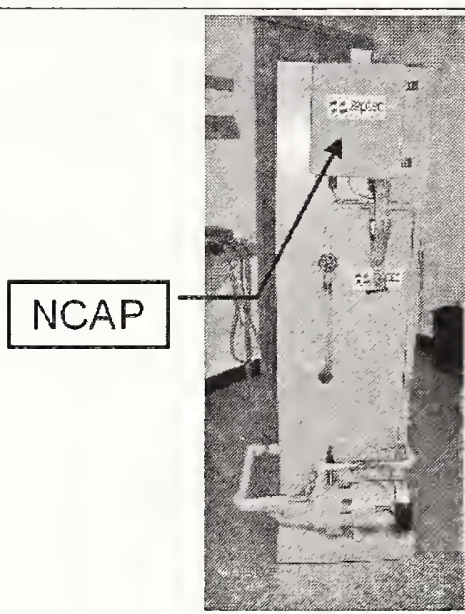




\section{NCAP Architecture}

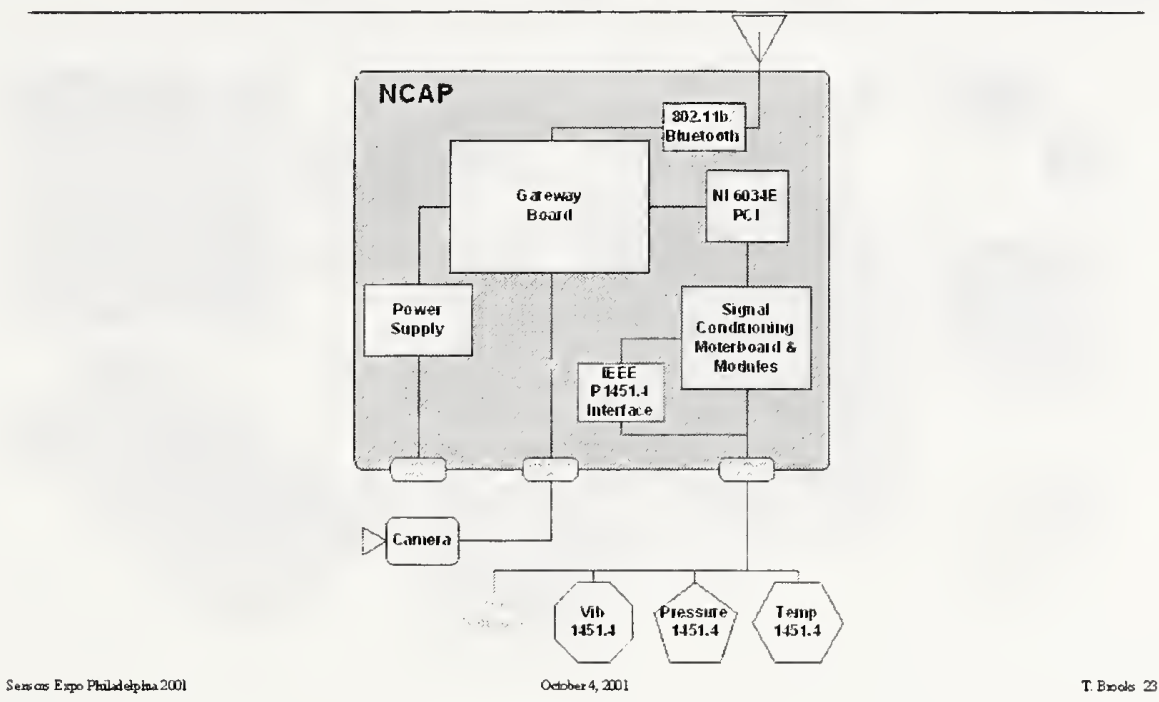

\section{NCAP Block Diagram}

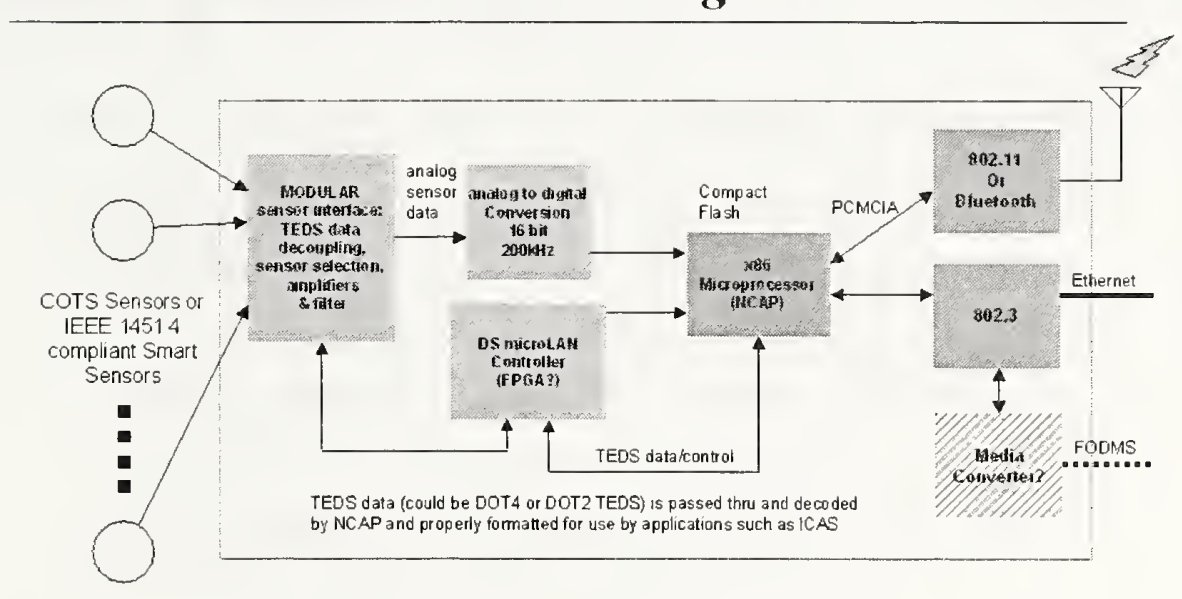




\section{Bluetooth Today (242 products)}

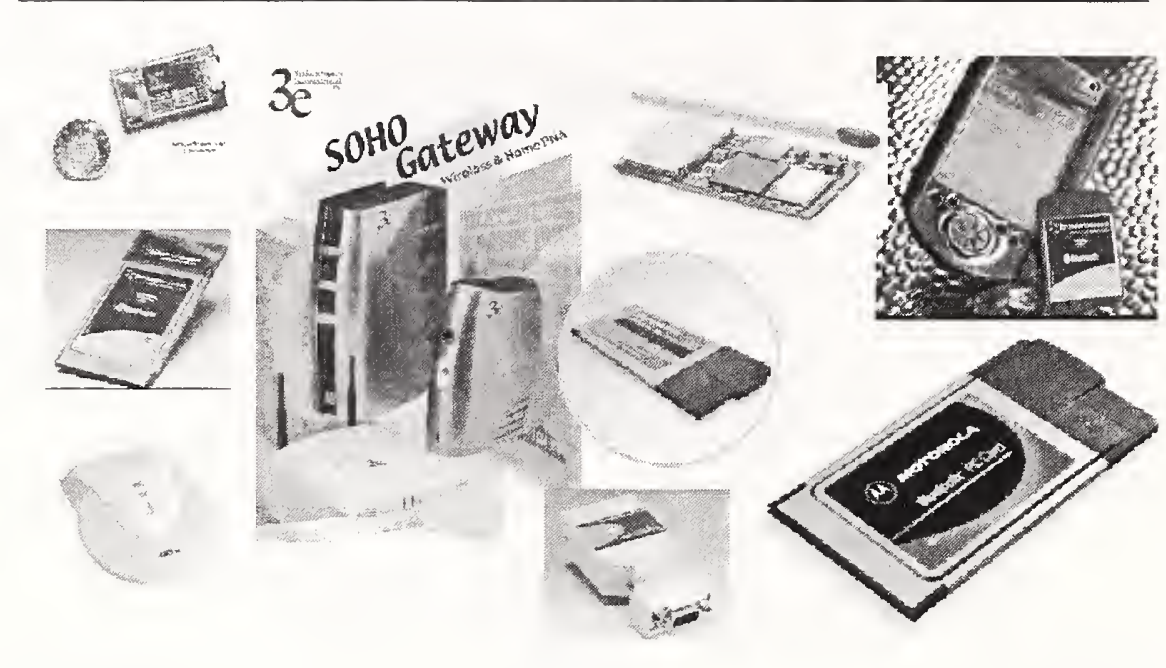




\title{
4.3 Synopsis on Wireless Ethernet (IEEE 802.11)
}

\section{Synopsis on Wireless Ethernet (IEEE 802.11)}

\author{
James D. Gilsinn \\ National Institute of Standards \& Technology \\ Intelligent Systems Division \\ jamesagilsinn@nist.gov
}

- IEEE 802.11 is an extension of the Ethernet standard (802.3) into wireless communications

- Allows roaming computers to talk to other devices (peer-to-peer) or connect to a wired network (transmitter receiver)

- IEEE standard allows interoperability between multiple vendor"s products 


\subsection{Network Examples}

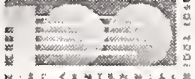

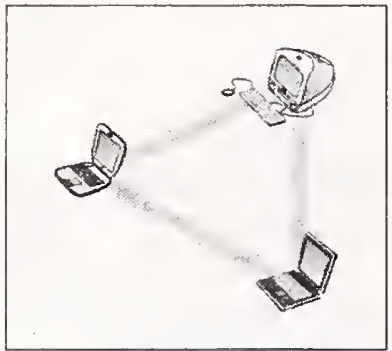

Peer-to-Peer Network

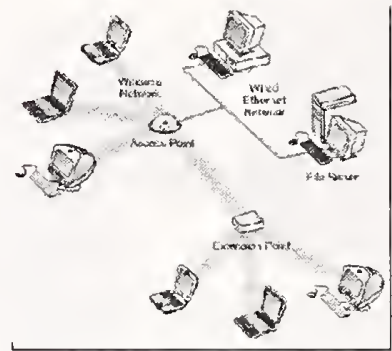

Transmitter Receiver (Wired/Wireless Network)

Pictures from Vicomsoft Web Site, http: //Frowe vicomsoft.com/

\section{IEEE 802.11 Specification}

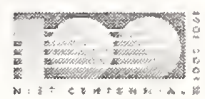

- Original specification 1997. revised 1999

- Speed: $12 \mathrm{Mbps}$

- Operating Range: $10-100 \mathrm{~m}$ inside, $300 \mathrm{~m}$ outside

- Power Output: $100 \mathrm{~mW}$ typical

- Frequency Hopping (FHSS), Direct Sequence (DSSS), Infrared (IrDA)

- Networks are NOT compatible with one another

- Uses $2.4 \mathrm{GHz}$ ISM band (2.402-2.480 GHz) 


\subsection{Variations}

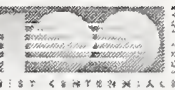

- IEEE $802.11 \mathrm{a}$

- Speed: 6-54 Mbps

- Uses $5 \mathrm{GHz}$ ISM band (5.15-5.35 GHz)

- Standard approved in 1999. but chip-sets and products only just starting to emerge

- Recently selected by the Dedicated Short Range Communications (DSRC) Vendors ${ }^{\circ}$ Consortium as the preferred technology to provide the national interoperability for Public Safety based applications

\subsection{Variations (cont'd)}

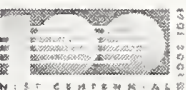

- IEEE $802.11 \mathrm{~b}$

- Speed: $5.511 \mathrm{Mbps}$

- Direct Sequence Spread Spectrum (DSSS) only

- Backward compatible with 802.11 devices using DSSS

- Most common implementation of wireless Ethemet

- IEEE P802.11e

- Make Quality of Service (QoS) enhancements

- IEEE P802.11f

- Improve the interoperability of Access Points 


\subsection{Variations (cont'd)}

- IEEE P802.11g

- Derelop higher speed extension for $802.11 \mathrm{~b}$ standard

- Speed: > $20 \mathrm{Mfbps}$

- IEEE P802.11h

- Enhance the 802.11 and 802.11a standards to enable regulatory acceptance of $5 \mathrm{GHz}$ products

- IEEE P802.11i

- Originally part of P802.11e

- Improve the security and anthentication mechanisms

\subsection{Variations (cont'd)} 6.54 Mbps Extengr on intelligent Systems Division $802.11_{g}$

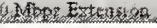

$$
802,11 \mathrm{~h}
$$

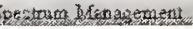

.

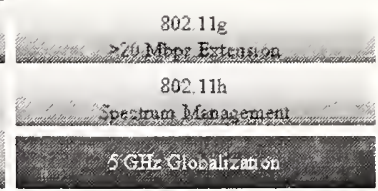

8 


\subsection{Security}

- IEEE 802.11 uses Wired Equivalent Privacy (WEP) algorithm to prevent eavesdropping

- WEP algorithm is self-synchronizing

- 64-bit key (40-bit secret code, 24-bit "init" vector)

- 128-bit keys seem common in production devices

- Data integrity checked with 32-bit cyclical redundancy check (CRC-32)

- Can be implemented in hardware or software

\subsection{Security (cont'd)}

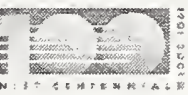

- Uses the same key to encrypt decrypt message

- This is a known security problem

- 64-bit WEP has been cracked!

- Multiple groups have reported being able to crack the secret code from a 64-bit WEP network in 15 minutes

- It is unclear whether the 128-bit encryption would provide much better security!

- Like wired networks. other precautions may be required to ensure data security 


\subsection{1 and Bluetooth}

- Tests have shown significant affects of having both IEEE 802.1 lb and Bluetooth devices in close proximity

- Good results as long as Bluetooth devices are kept $10 \mathrm{~m}$ away from the 802.11 Access Point (AP)

- Worse results when Bluetooth derices are near Station

\section{Summary \& References}

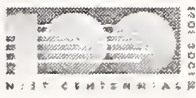

- 802.11 provides

- Flexibility

- Interoperability

- Upgradability

- Ease of Use

- Although it has its drawbacks, 802.11 may provide a good extension to industrial Ethemet
- http//vmw. ieee org

- hitp//nтrw wlana com

- http//nww wirelessethernet org

- hittp//rww itsa.org

- http//wnw zdnet com

- http//nrwintenetnews com

- littp/awww orellynet.com

- http//www viconsoft con

- hitt//www atheros com

- http/anww proxim com

- http/ кxww ciscocom 


\subsection{Reliable Wireless Connectivity for Sensing \& Control Applications}

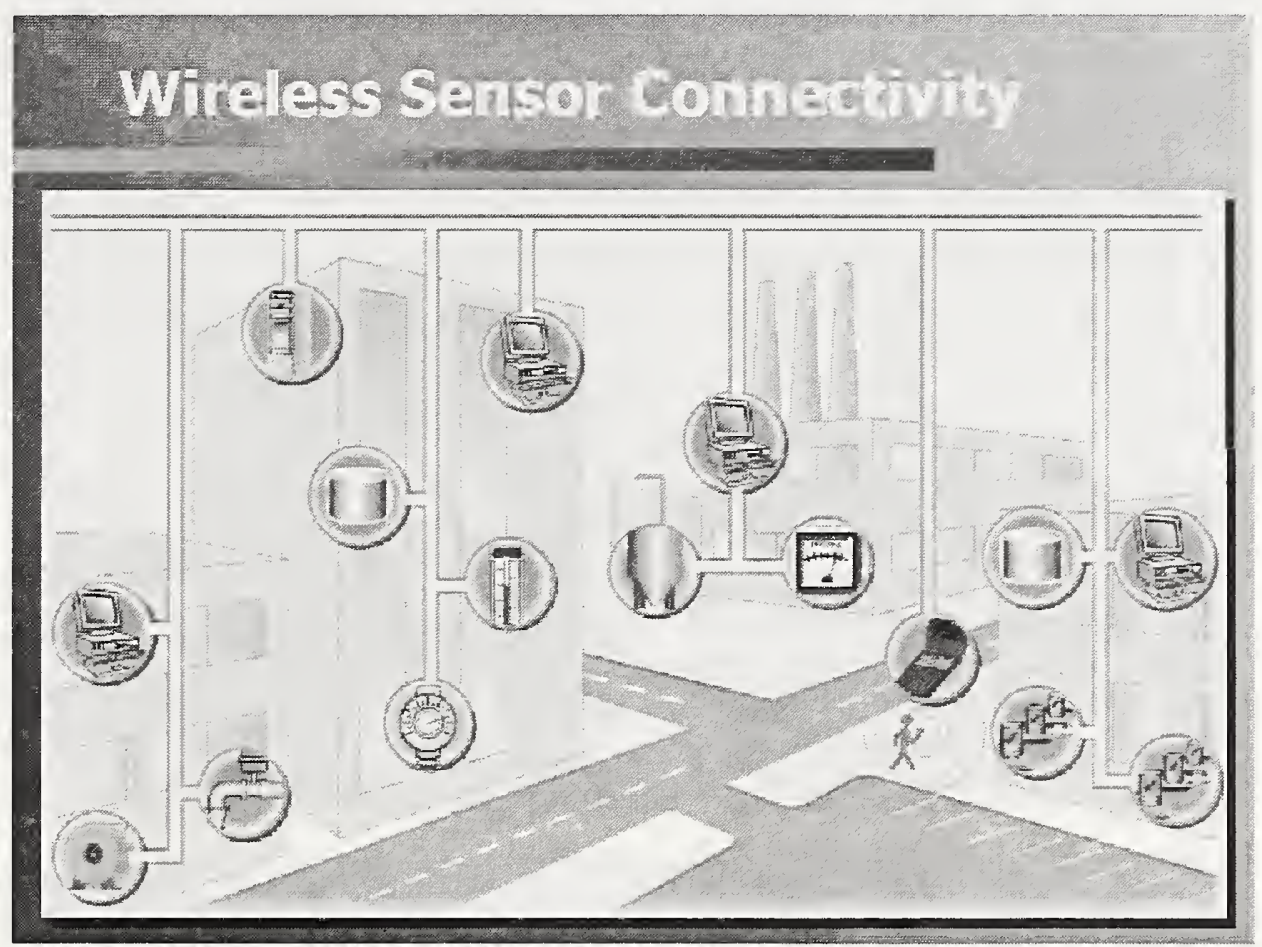

\section{Indushn guores}

"It's not going to be peoplen, its gotng to be sensors, mierocontrollers. Thilions of these will be connected to the in ternet"

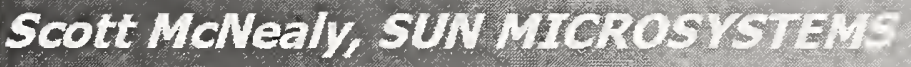

"... there will be a day when a person malses use of tens of hundreds of telemetry devices per day without even knowing it."

THE YANKEE GROUP REPORT

AKOANN 


\section{Industry Quotes}

"Everyday devices ... will be conneated [to the Internet], resorting on their usage and status. Each of these speplications of dialtil information is approading an infiection point aw

\section{BHI Gates, Michosoft}

\section{AKôN}

\section{Wreless sensing: costbenetis}

Wife Installetion Cost: $\$ 20-\$ 50$ pr rook, nors hil $30 \mathrm{~ms}$ environments

- Oren too expenste bo wins - dats is lost, efflciencies are imper ad

-Wireless is easy to instoll 


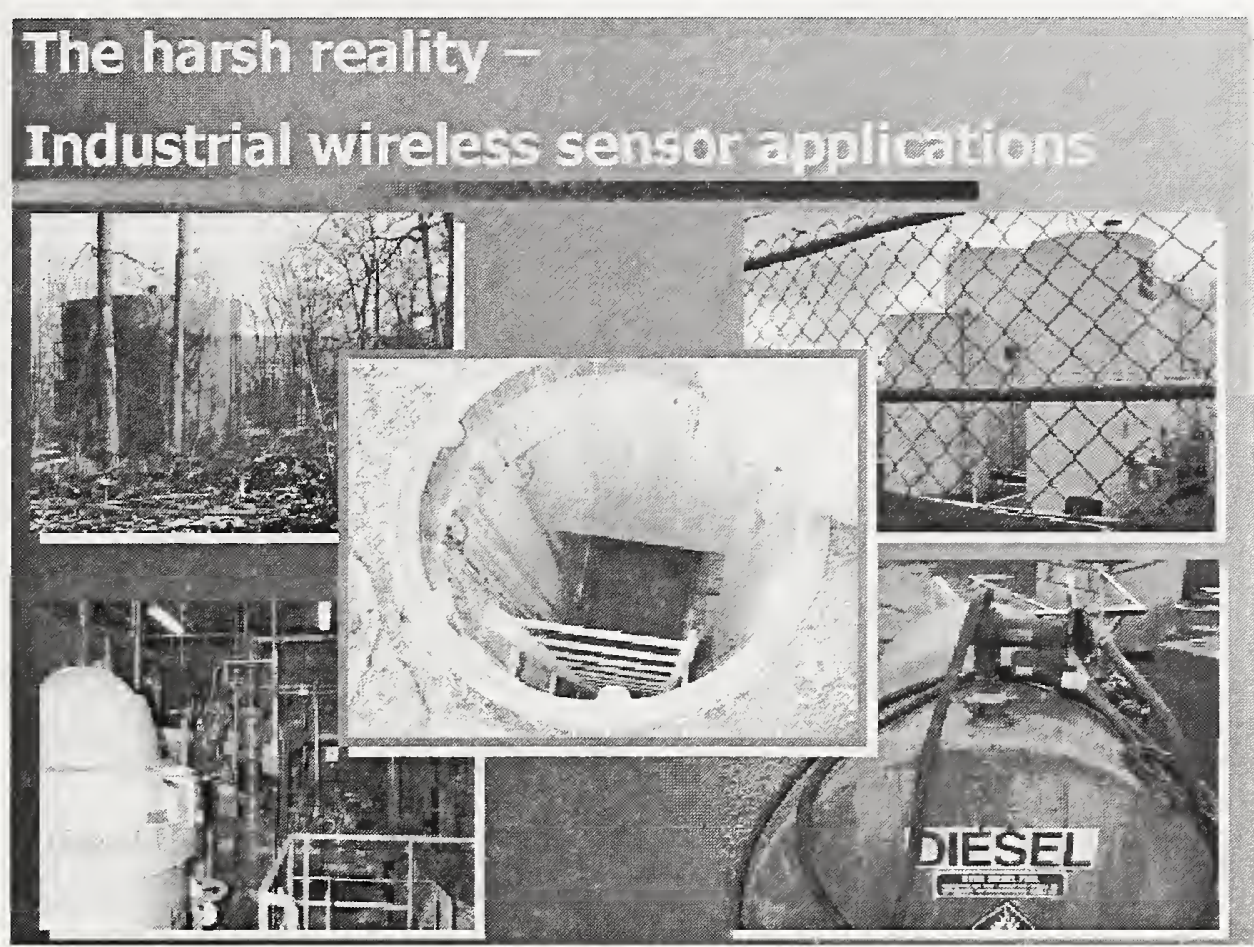

\section{Applications}

Process control

- Oil 8 gas prodiction

- Manufacturing

- Petro-chemical : refineries

- Industrial sensors: temperature, pressure, jow fevel

- Energy management

- Tank level monitoring

- Waste water management

- Utility AMR 


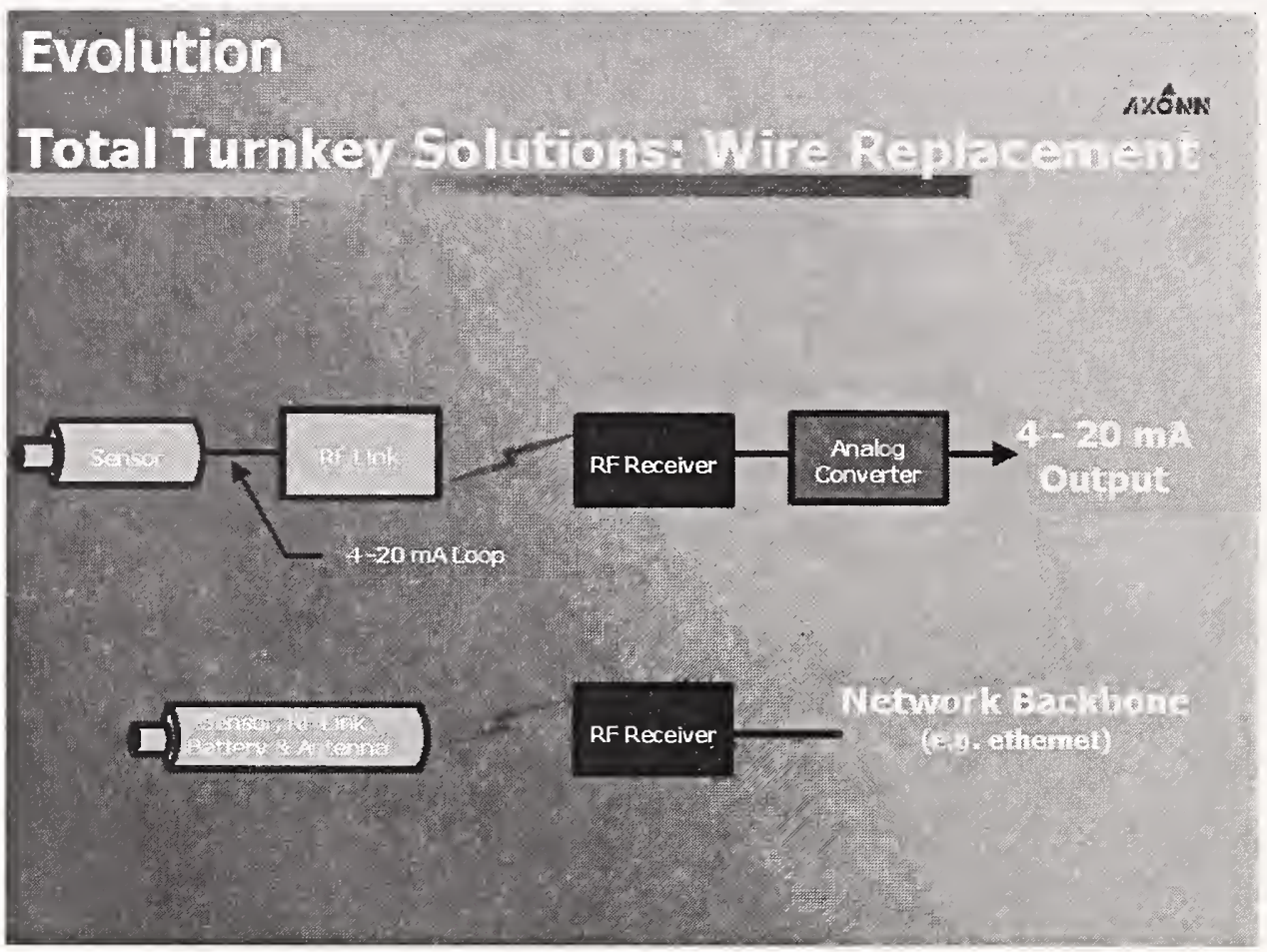

ARON customer limplementations nxôw

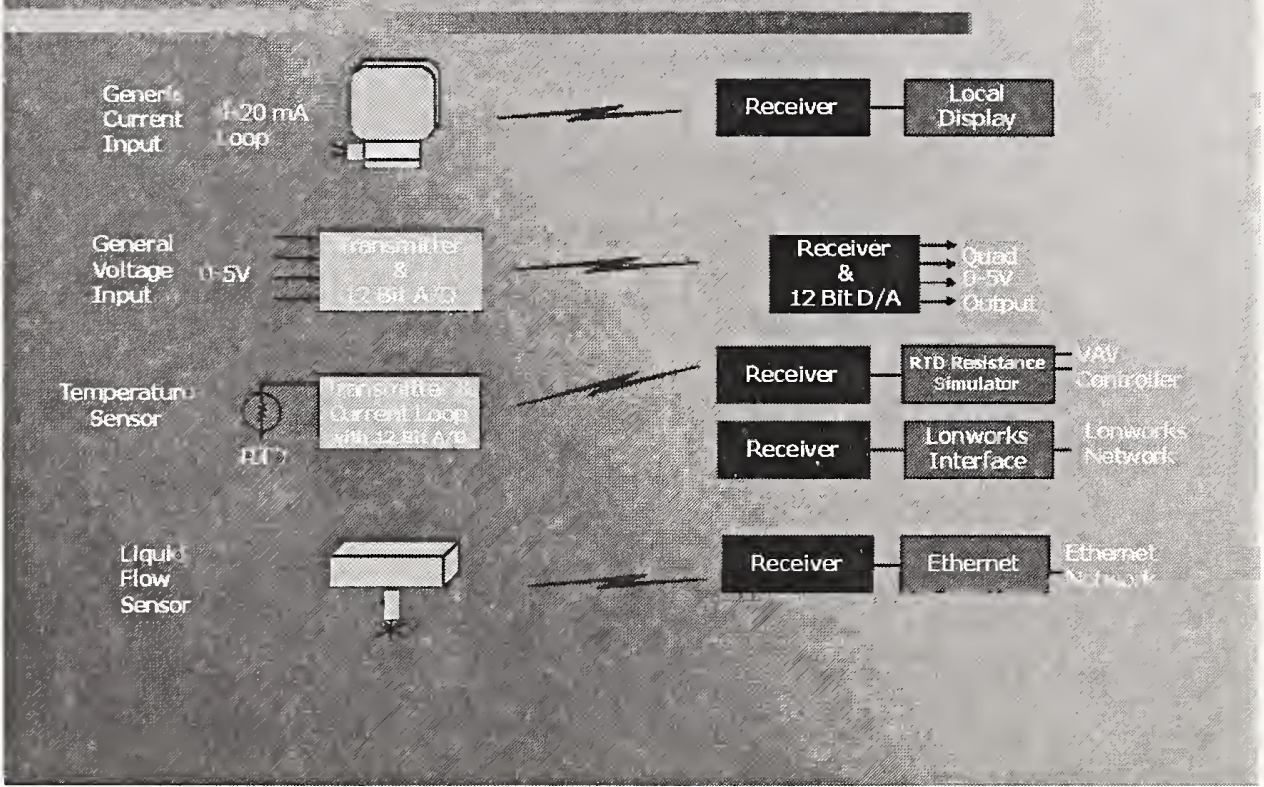




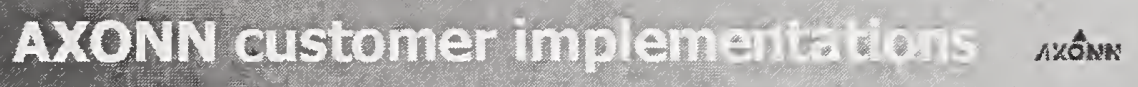

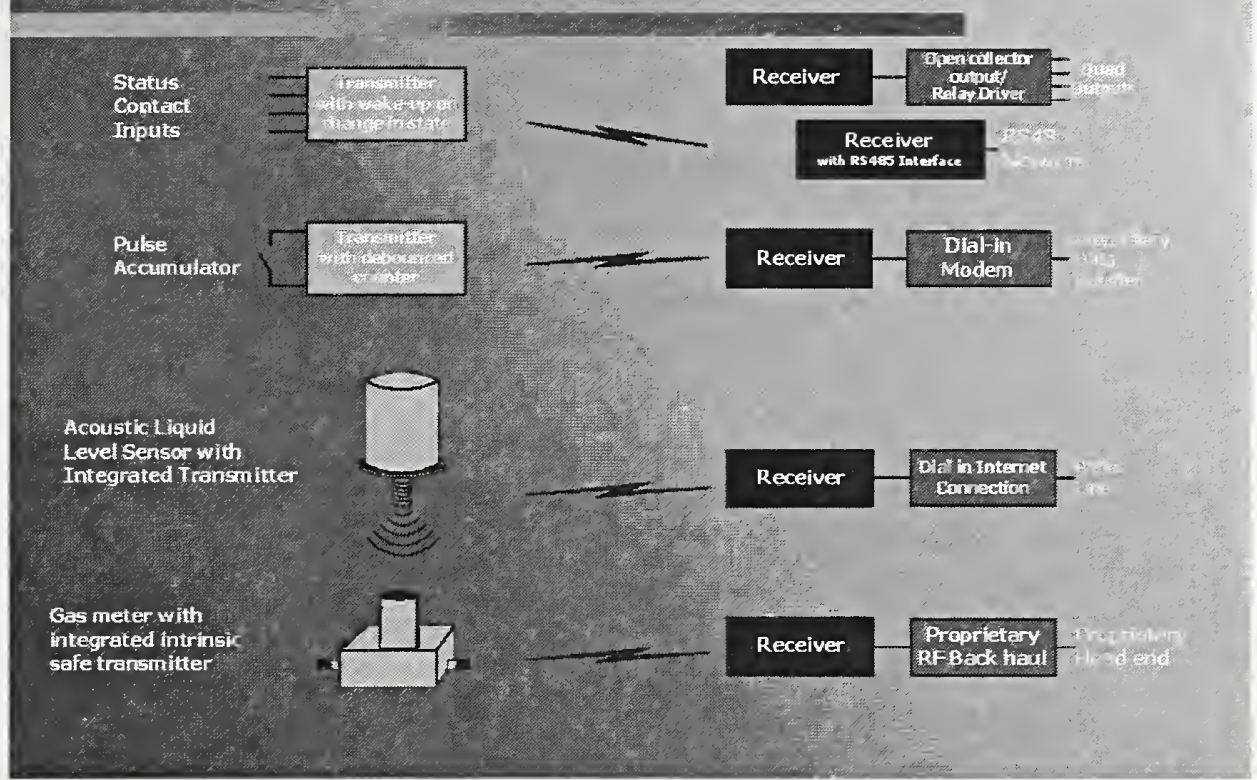

\section{Data rate ve range}

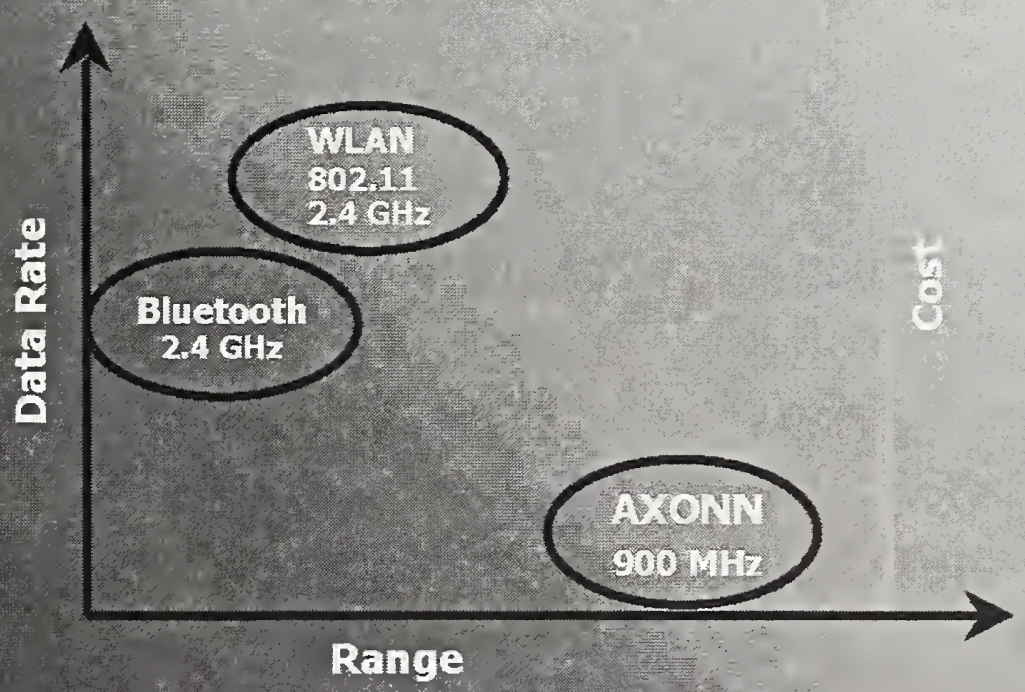




\begin{tabular}{|c|c|c|c|}
\hline \multicolumn{4}{|c|}{ 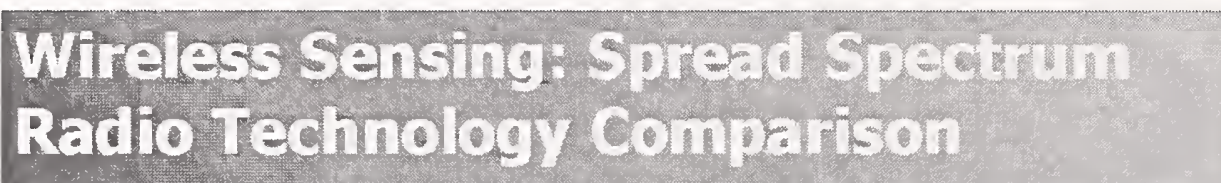 } \\
\hline Teahnology & $3 \pi N T=302,119$ & Bluetorith & Grom soumte \\
\hline Frequencr & 2hrent & 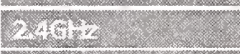 & grovites \\
\hline Ss Modulation & DS56 & $x^{-15}$ & B8s: \\
\hline Data Rate & Whol 5xit Mires & High mibes & bow sysente \\
\hline Reaelver Sensitivity & Vancris & $=213 \mathrm{Bm}$ & 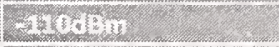 \\
\hline $\begin{array}{l}\text { Radio Range } \\
\text { (maximum: RXat BW }\end{array}$ & 100 hymon & cictormin & 7exmils \\
\hline Temp Range & $\mathrm{ma}=0$ & Non & 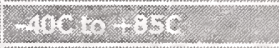 \\
\hline Band Crowding & Hight & Sigliz & Mtinn \\
\hline $\begin{array}{l}\text { Bulding Penetration \& } \\
\text { Propagation }\end{array}$ & $10 x y$ & leot & feriting \\
\hline Standardized & Yes & $\operatorname{sen}$ & 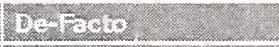 \\
\hline ISN Band & res 20100 & 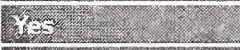 & 65 \\
\hline $\begin{array}{l}\text { Multipoint Coexistence } \\
\text { Capebility }\end{array}$ & lon 20 & VtIm & hish \\
\hline Vendor Diversity & 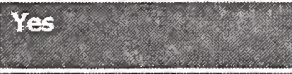 & Yes & 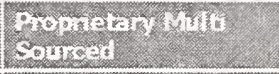 \\
\hline Target Application & Wricless Fitiemet & ems Lisully & 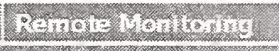 \\
\hline
\end{tabular}

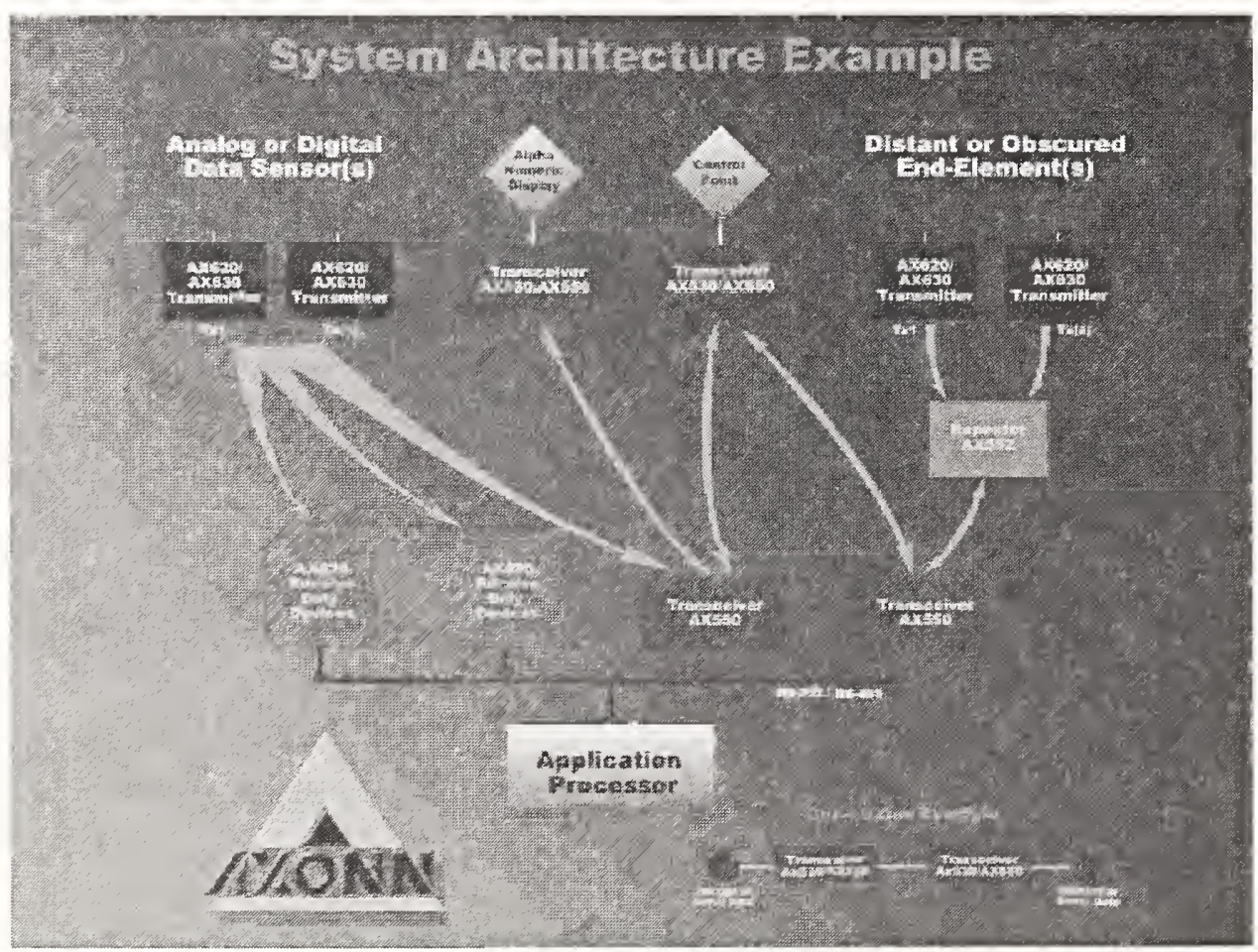




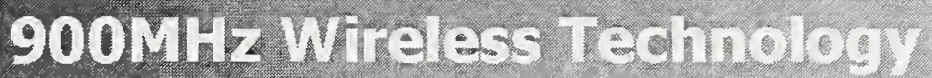 Highlights}

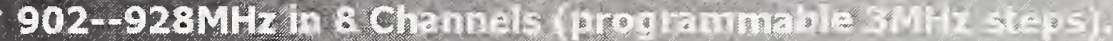

- CDMA \& TDMi operator.

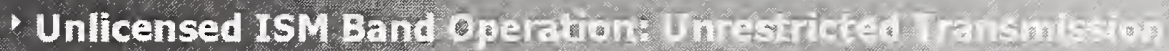
(update) Rete

- FCC Approved Now

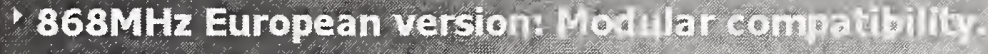

50-250mW output porter

1 mile range (LOS).

1 Receive Sensitivity - $110 \mathrm{dBm}$ a) $1902 \mathrm{kps}$ if th tate

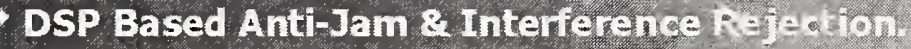

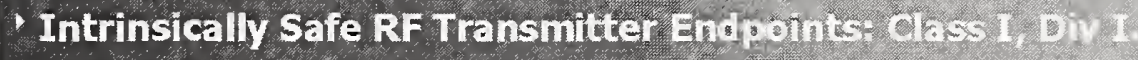

- Integrated Antenna (external ankenmac optional)

- Battery operated transmitters (Lifer maws for

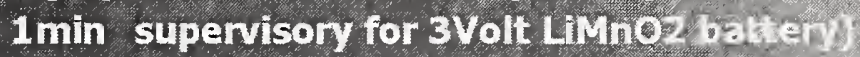

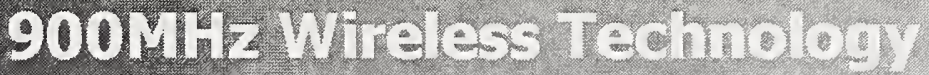 Highlights}

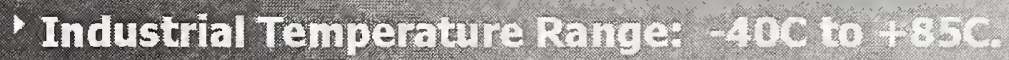

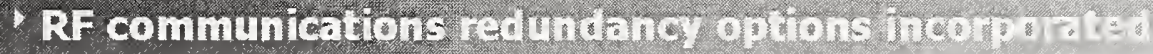

- Modular Radio, or Plug i play obrons

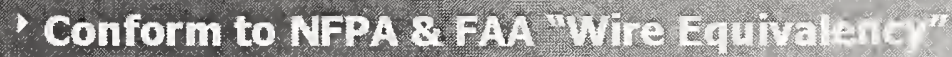

Requirements

Technology Foundatone

60 patents \& Patents Peiling.

Full Custom Mixed signal istes mum-sourced)

- Comprehensive Sygtem Arahit - gllt?

- Proven in the field now 


\section{Current high volume Tnotallations (roll-out phase)}

- Tank level monitoring

- Fire \& security

- Utility (AMR \& load/Aistribution control)

- Energy management Bt sub-metering

- Waste water management

- HVAC control \& commeroil bufiding controls

- More than $6,000,000$ devices in field operation

AKớn

\section{Beta instillatons}

- Oil a gas provuraton

Transportation monitoning (railear)

- Pressure Sensing

Parking meters

- Fleet monitoring (assot tracking)

vending 


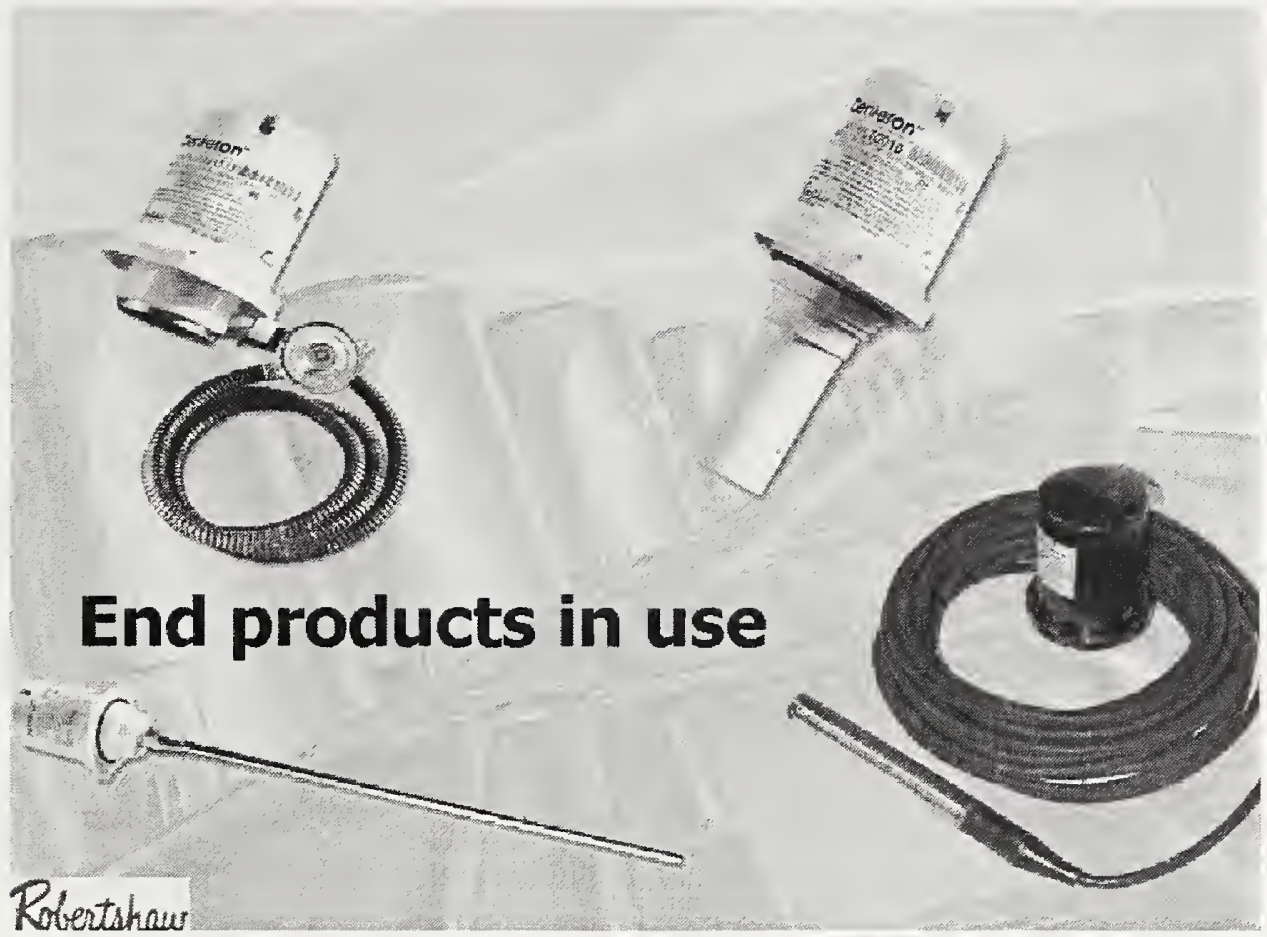

\section{End Products:}

Industrial wireless sensor applications

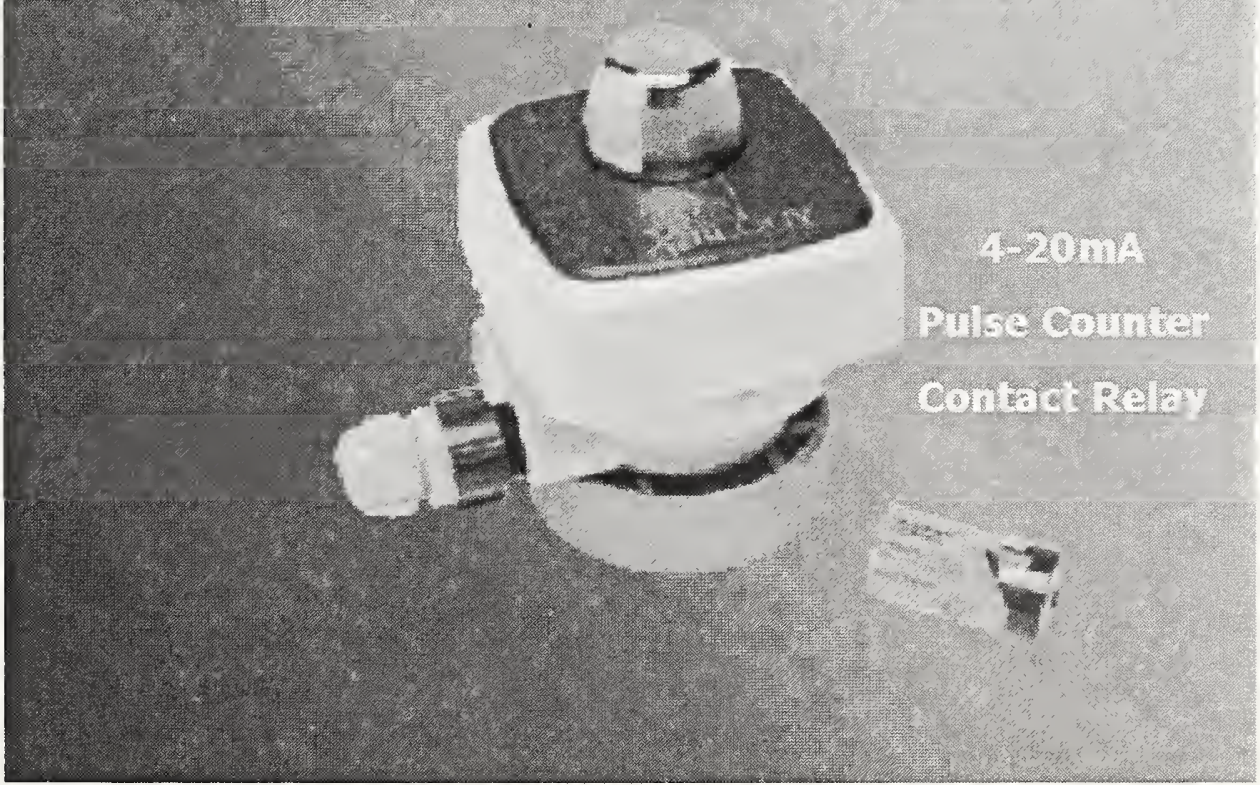




\section{End Products:}

Industhtal wireleso sensor appliter itons

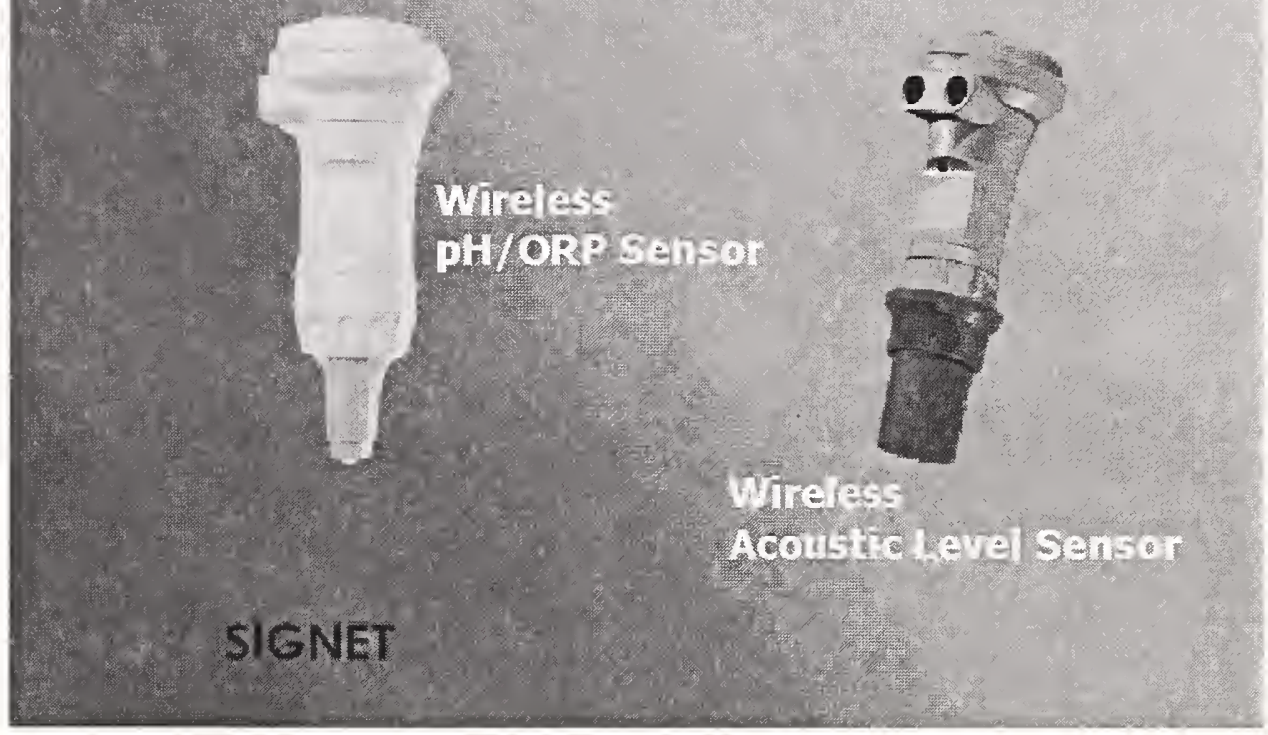

\section{End Prodtcis:}

\section{Uifts \& energy managevent} appliterions

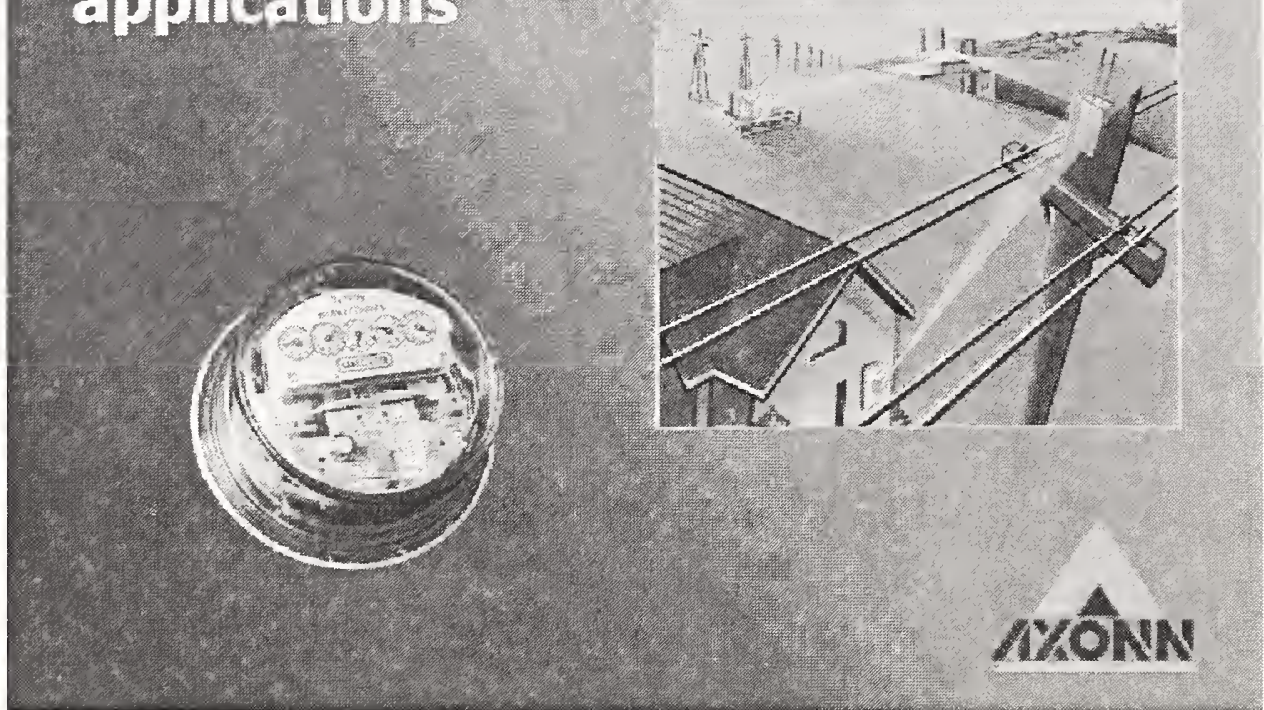




\section{Battery life is power consumption}

BuA sleep current (stil operaung oscillator and monitoring lipitis

Uses small (2/34 sire) $\cos 2$ cheap camera batteries

plyzas zyour

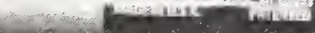

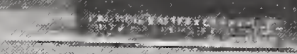

No external power required for transmitters

Battery status is sent in each transmitted message

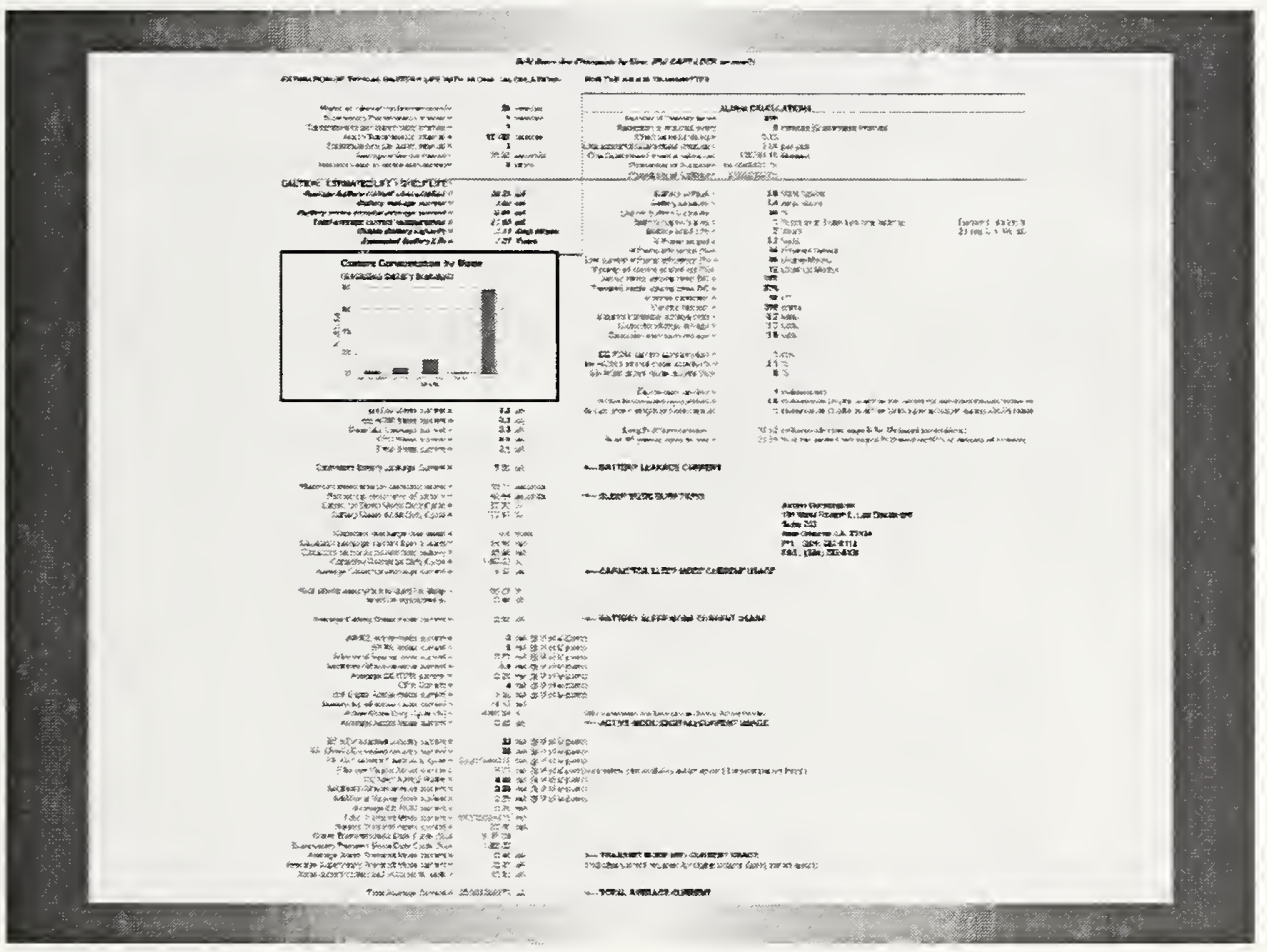



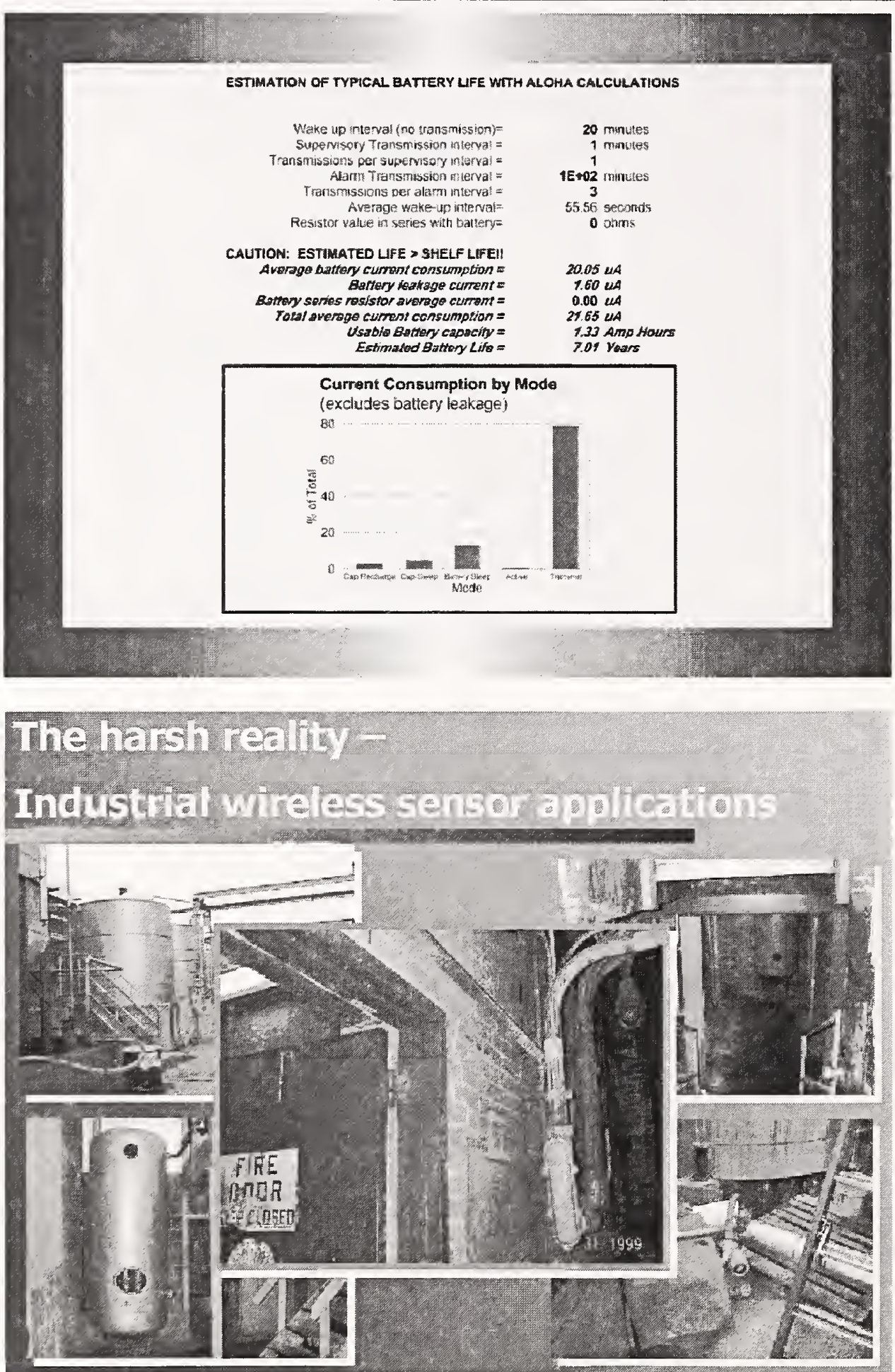


\section{Envinomenta Dograles

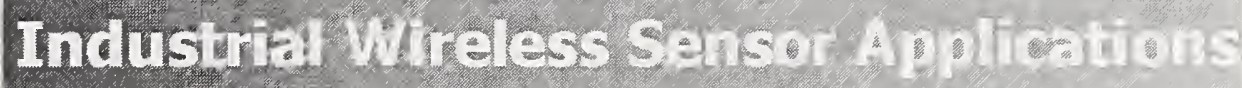

\section{$\mathbf{R F}$}

Interference

Woot 20

Brick

$32 \%$

\section{High Hotse}

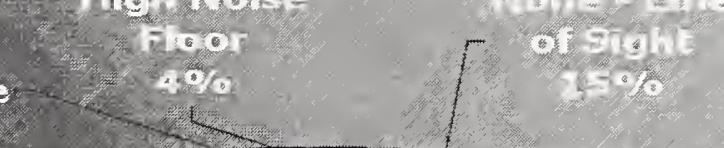

Follage

$10 \%$

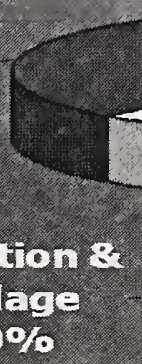

Sourcet X silogy Inc

\section{Range - Outoors}

AKỐN

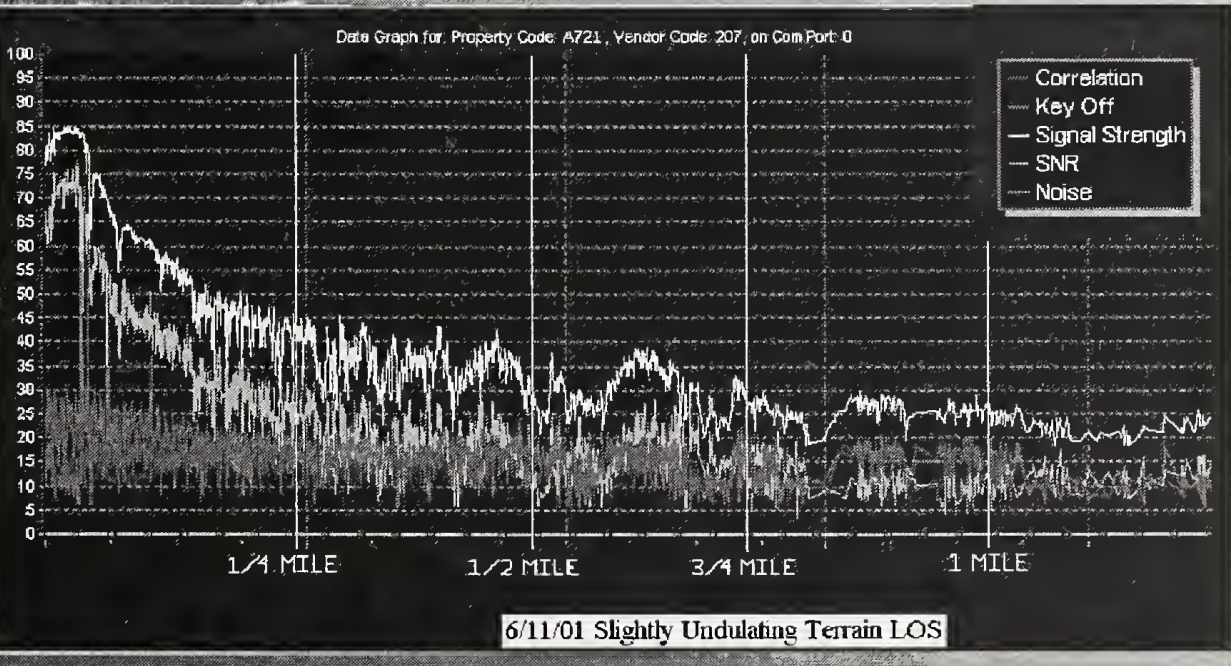




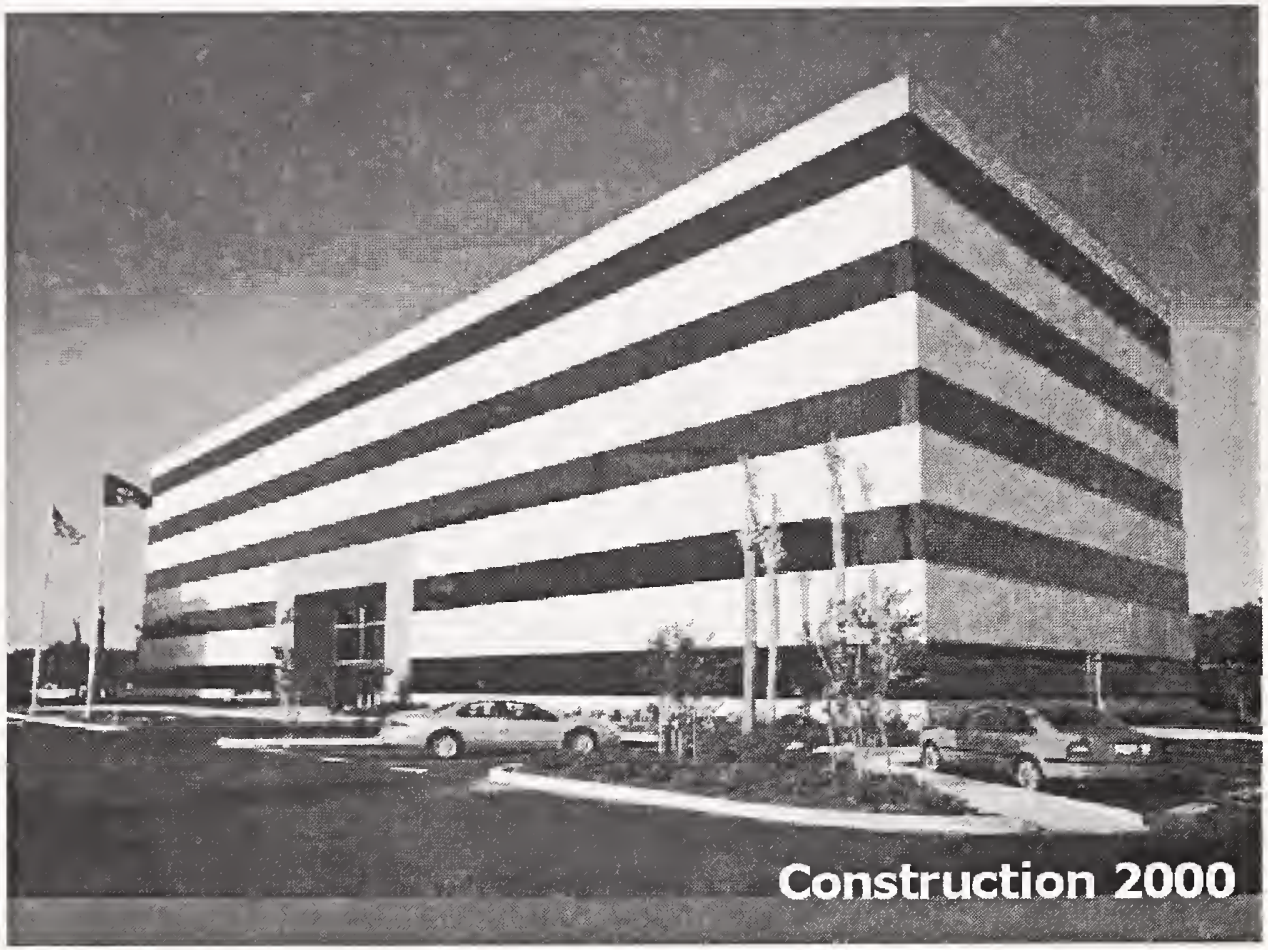

\section{Range Indoors}

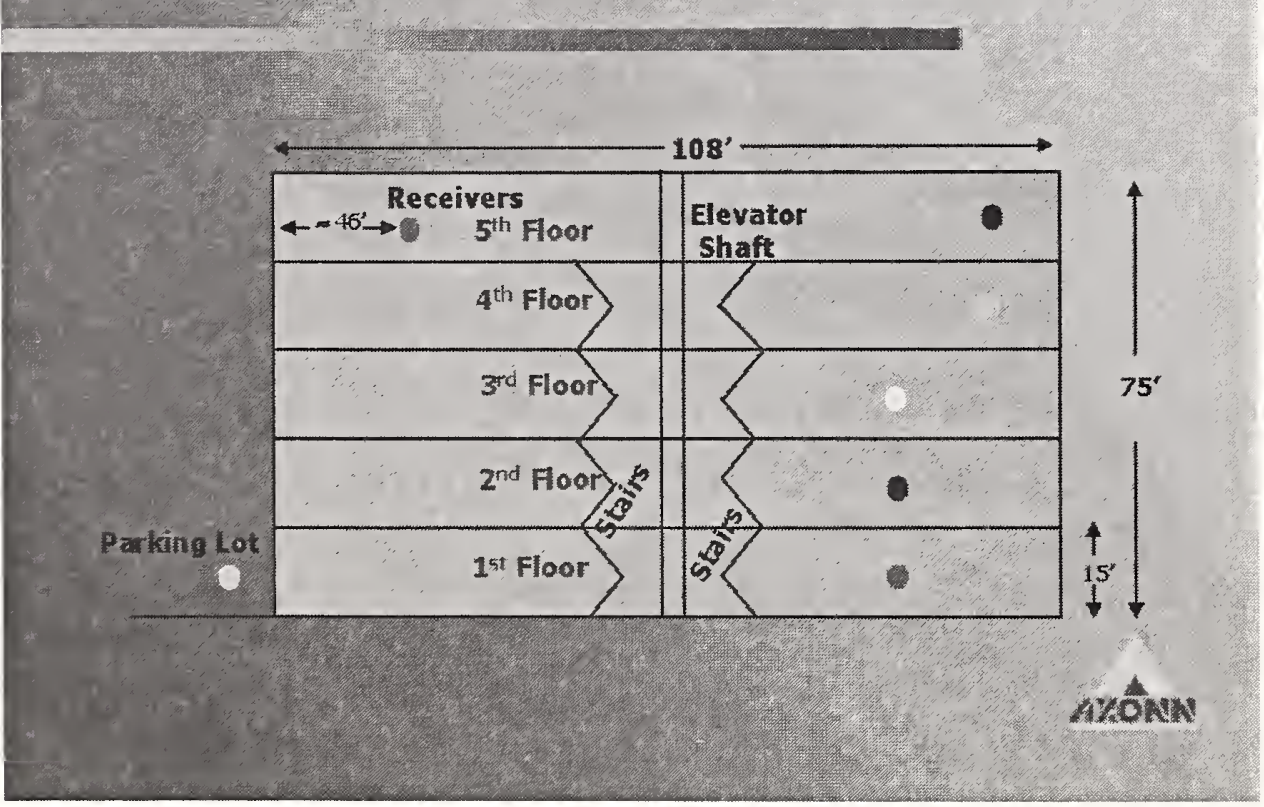




\section{Reliability Operation}

- Operating Temperature Range

$-40 \mathrm{C}$ to $+85 \mathrm{C}$

- Intrinsic Safety Class I, DIV I

Needed for many sensing end-point applications

nxợw

\section{Reliability: RF Communication}

Multiple redundancy approaches used for RF communioation relability

$\checkmark$ Redundant transmissions:

Burst of transmiserons randomly gresed in tithe

$\checkmark$ Redundant receivers:

Overlapping coverage

$\checkmark$ Antenna Diversity:

Reduce fading effects

$\checkmark$ Frequency Diversity:

Multiple frequencies to Axốn circumvent jamming sources 

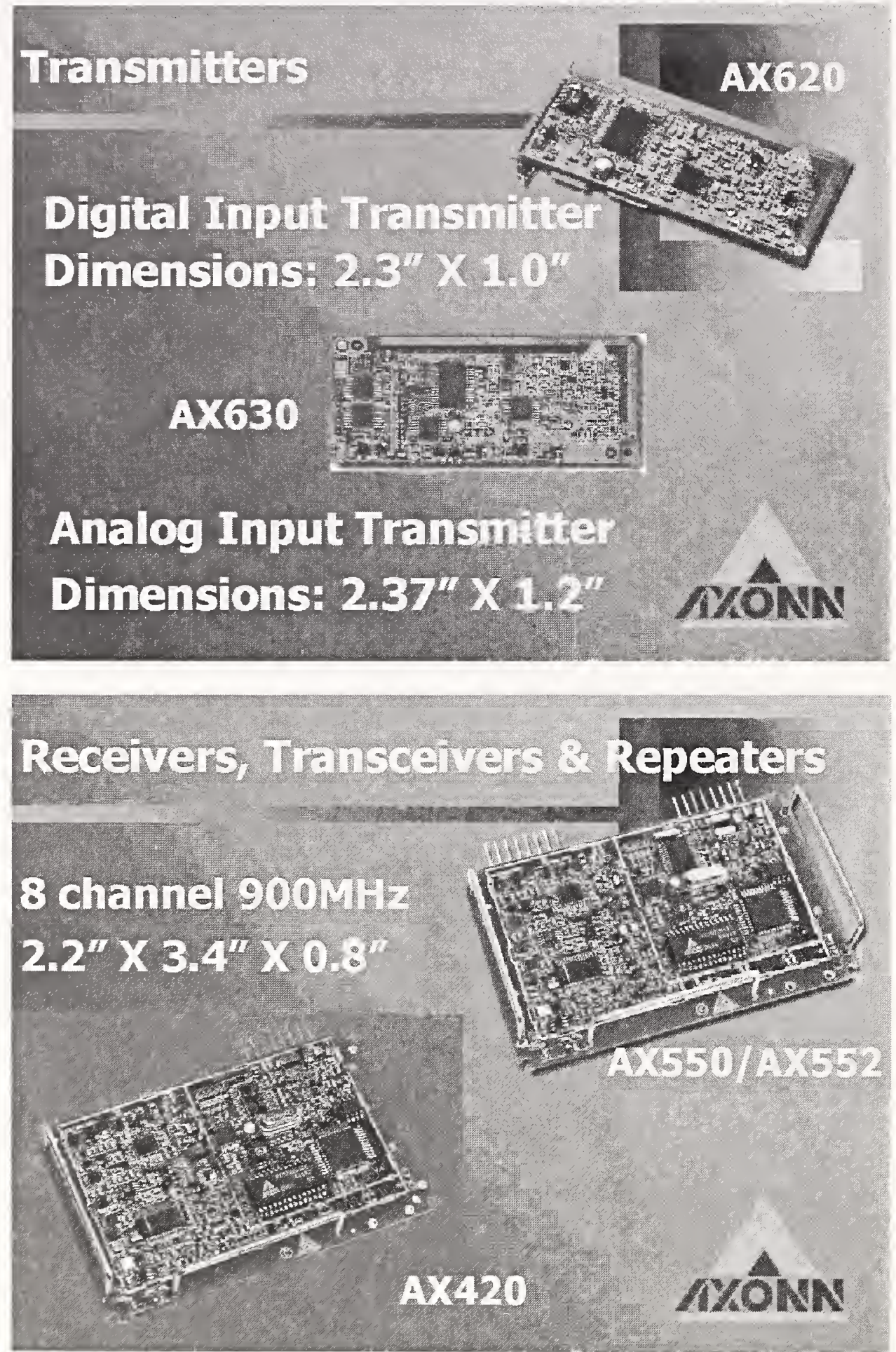

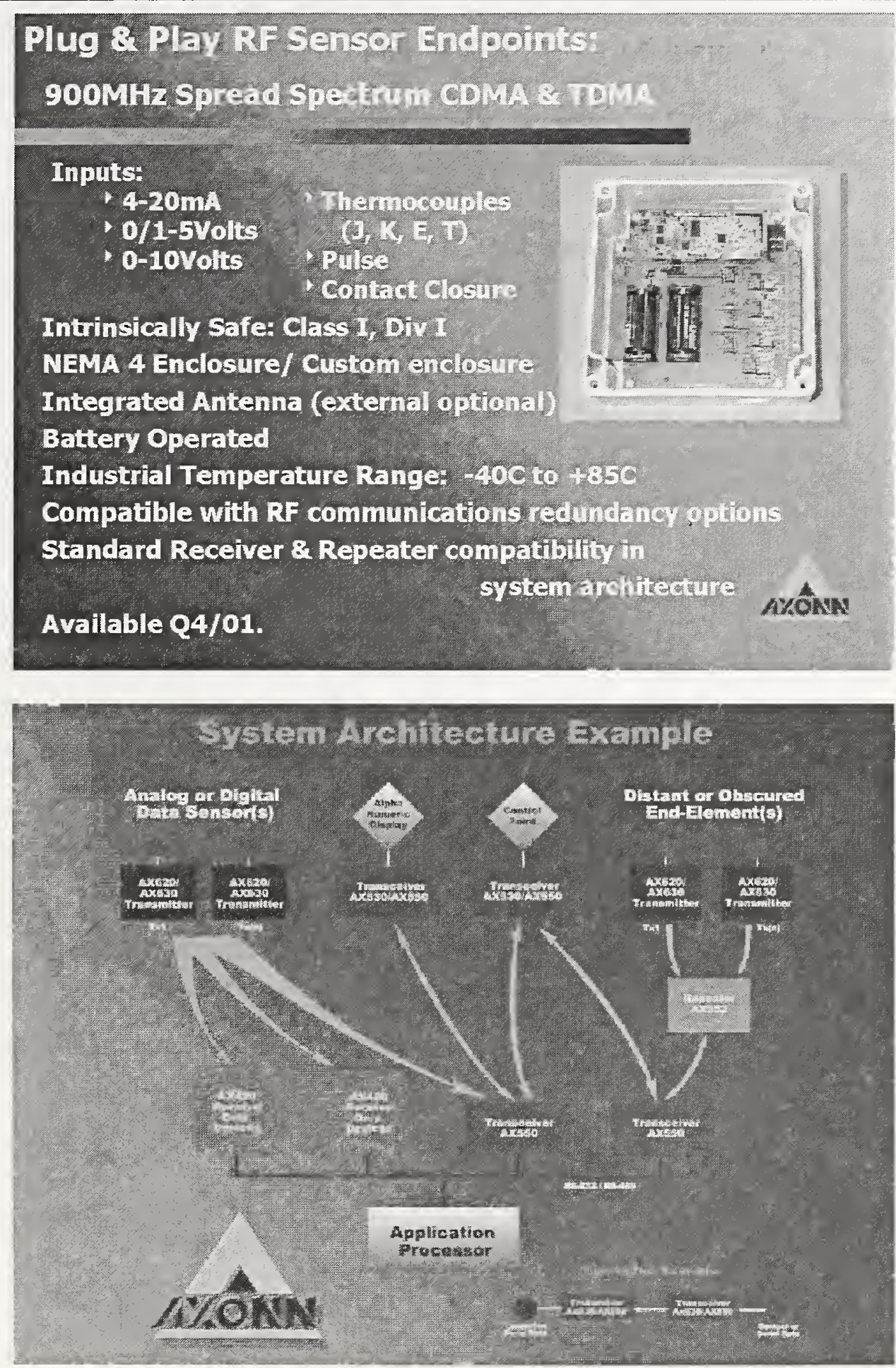


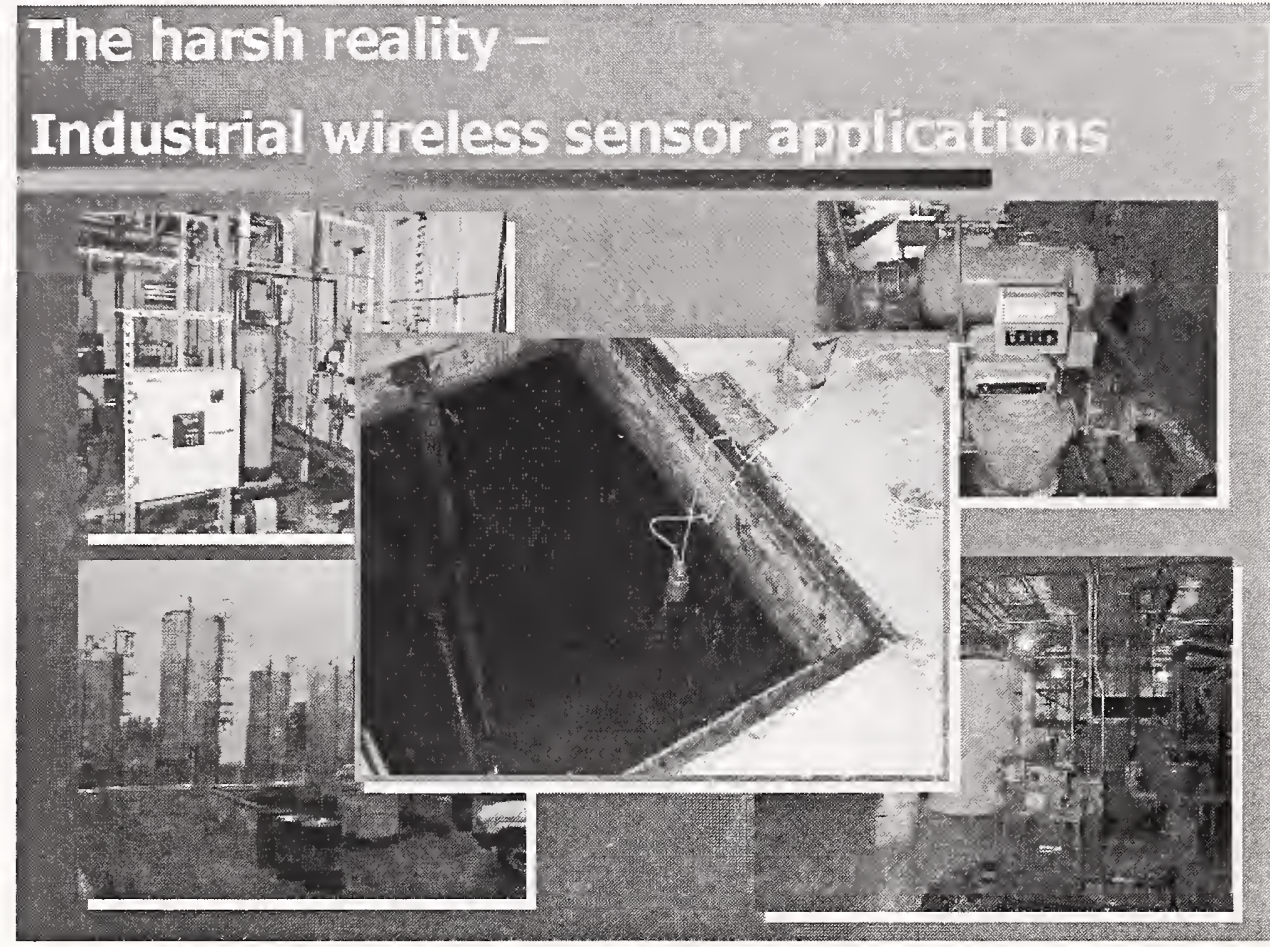

\section{OOMTFE}

- Leartiug provifer ot lo v cost spzea sosistim is technology

- Develouer bor tolto of 60 patents 8 patents ponding

- Placed aver 6 untiton devices in the ret

Founded in 1985
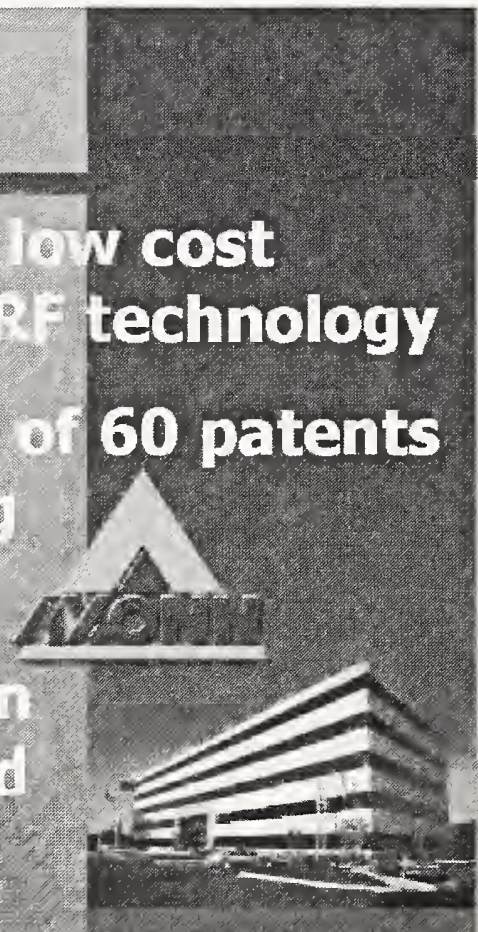
Key considerations in choosing a witelexos solution for sensing and eontrol a blications

- Wireless telemetry works - 6 milion tines and connting

- Wireless connectivit technology mist be matehed to application

- Economics will force pervasive wirelers senting in SCADA segments

Wireless will ultimately drye thto inaditonally hartwired solutions

It's more than margin enhancement wireless will camse transitions in traditional business proctrees (from fighting commoditization to winthy customer loyality) MONM

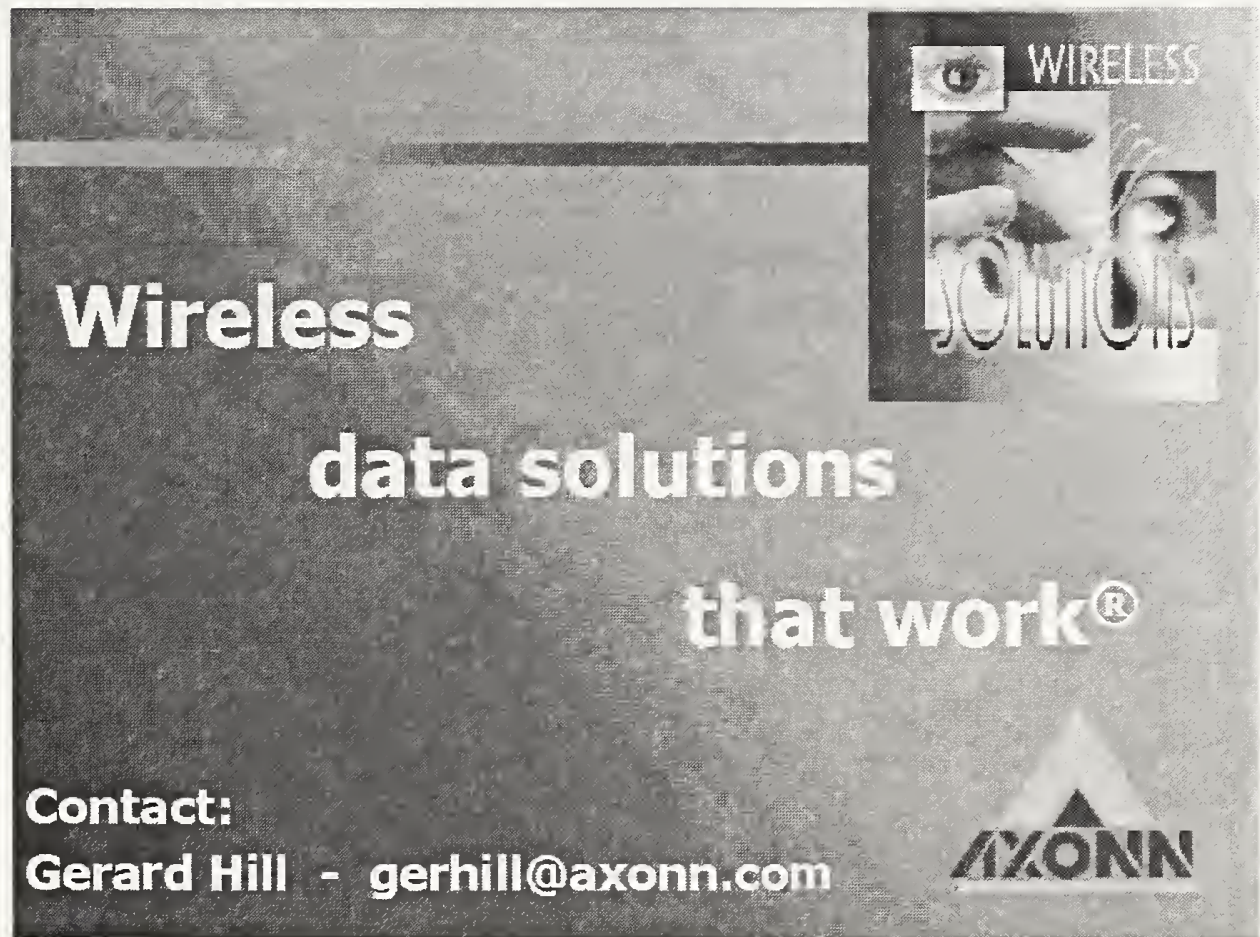




\title{
4.5 IEEE 802.15.4 Low Rate Personal Area Networks
}

NSF Sponsored Workshop on Tether-free Technologies for e-Manufacturing and e-Maintenance/Service

\section{IEEE 802.15.4 Low Rate Wireless Personal Area Networks}

\author{
Ed Callaway \\ Florida Communication Research Lab \\ Motorola Labs \\ ed.callaway@motorola.com
}

\section{IEEE What?}

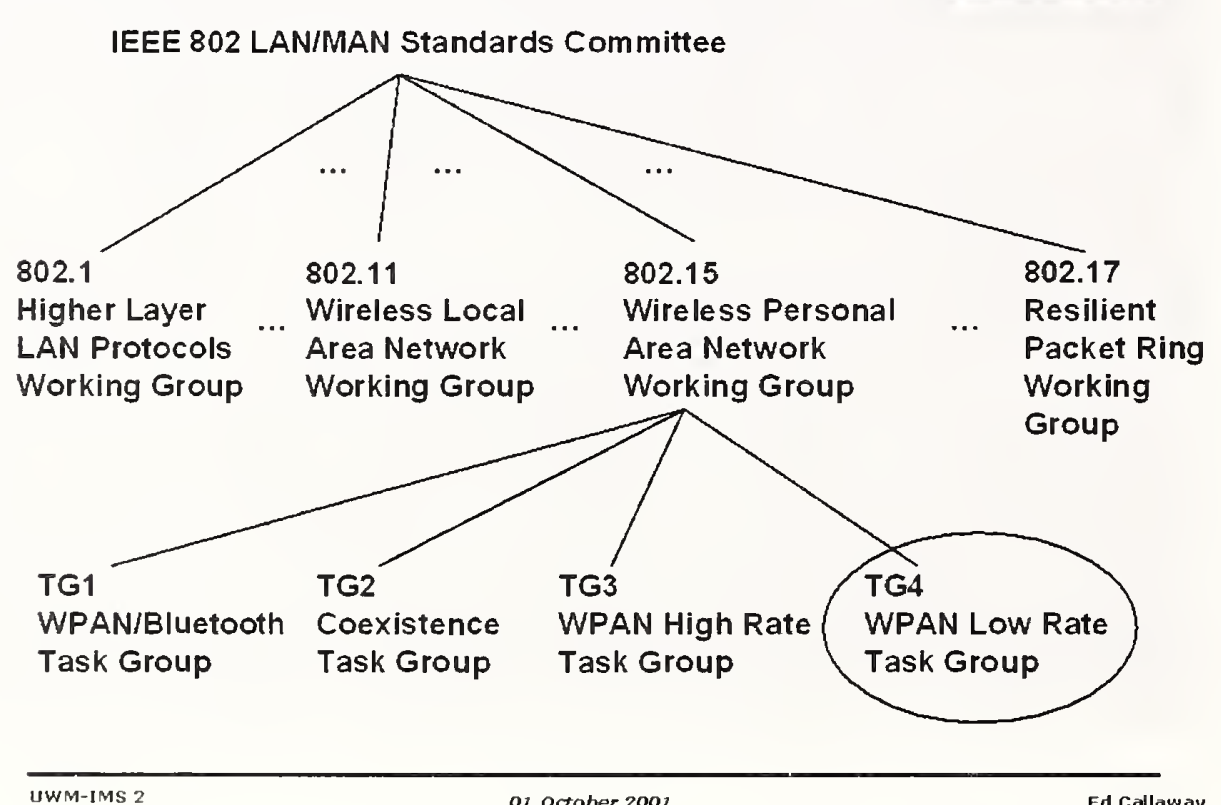


Now in Development ...

A low-rate wireless personal area network communications protocol that:

-Supports multiple network types,

-Has long battery life (months or years from a AAA cell)

-Is low cost

For systems with moderate data throughput $(<250 \mathrm{~kb} / \mathrm{s})$ and QoS requirements.

-Supports star \& peer-peer topologies

-Master/slave, point to any point, cluster tree, etc.

-Access is slotted CSMA-CA

-Data rates of $31.25 \mathrm{~kb} / \mathrm{s} \& 250 \mathrm{~kb} / \mathrm{s}$

-Optional use of network beacons

-Optional time slots for low latency transfer

-Super-frame is contention based 


\section{Node Types}

- Distribution node

- Controls the network topology at that node

- Master/coordinator or mediation device

- Stores routing information

- Talks to other distribution and slave nodes

- Slave node

- Cannot control the network

- Very simple implementation

- Does not store routing information

- Talks only to a distribution node

\section{Optional Super Frame Structure}

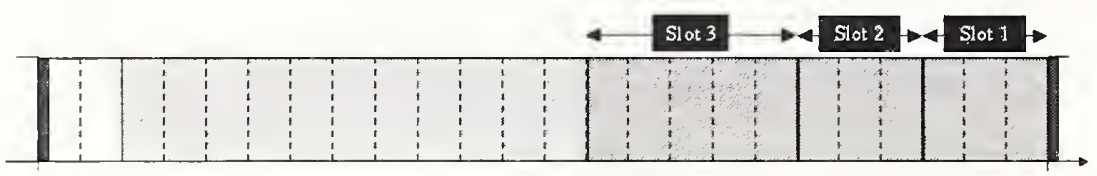

Netwod:

beacon

Beacon

extension period

Contention period

Allocated slot
Transmitted by distribution nodes. Contans neturost information. super frame stmichure and notification of pending node messages.

Space reserved for beac on grouth due to pendung node messages

Access by any node using CSMLA-CA

Reserved for nodes requiring guaranteed bandwidth 


\section{Master/Slave: Network Connection}

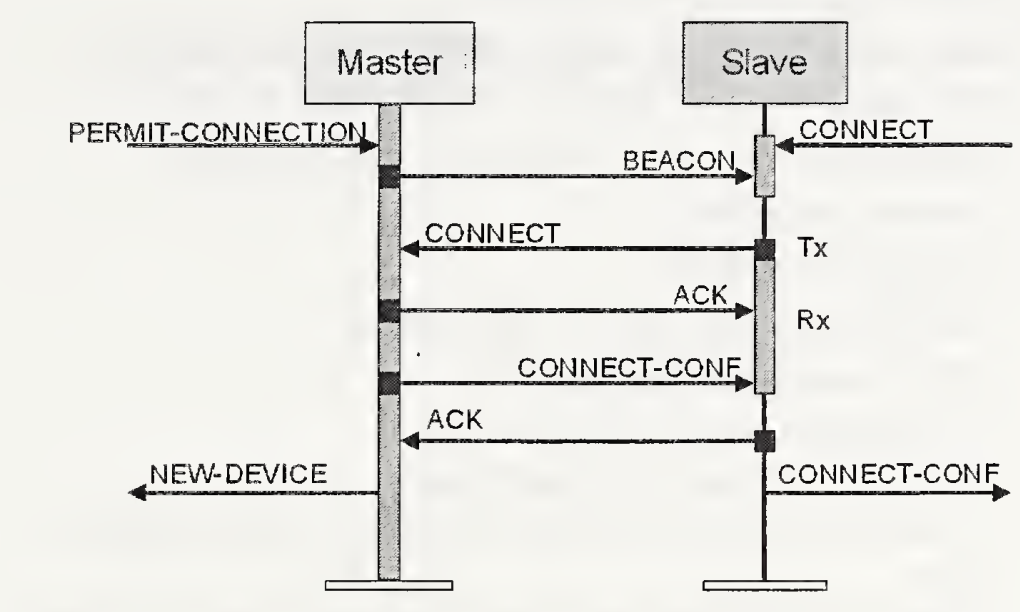

\section{Mediation Device Operation}

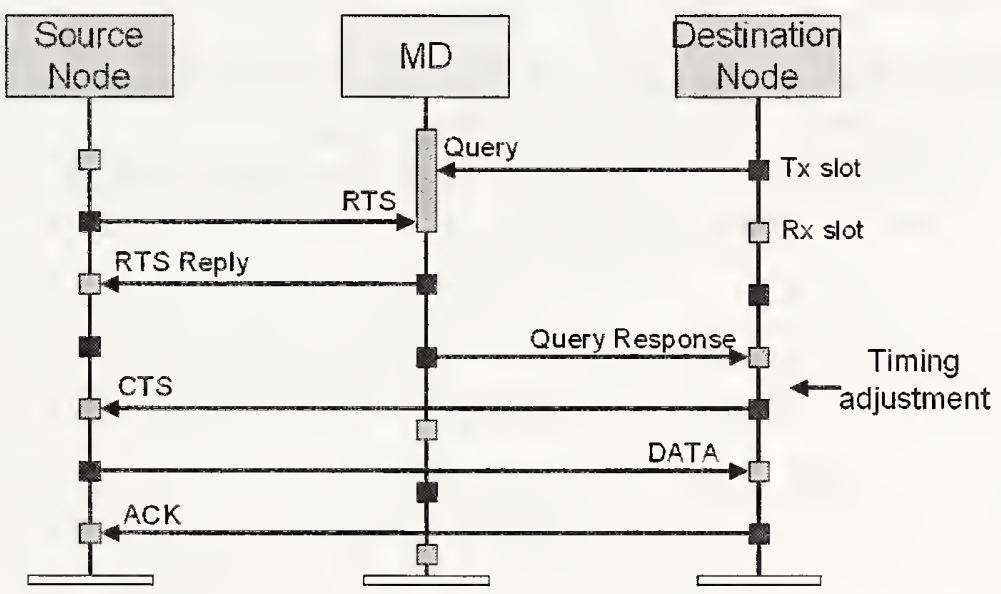

... Solves synchronization problem for low cost, low duty cycle peer-peer systems 
a 31.25 and $250 \mathrm{~kb} / \mathrm{s}$ operation

- DSSS with low chip rate $(1 \mathrm{MHz})$ for low power operation

- O-QPSK, for constant envelope modulation

- Simple, low-cost PA

- Orthogonal coding

- Greater range for a given output power

- $5 \mathrm{MHz}$ channel separation

- 16 channels in the $2.4 \mathrm{GHz}$ band

- 5 channels in the U.S. $915 \mathrm{MHz}$ band

- 1 channel (at lower data rates) in the European $866 \mathrm{MHz}$ band

- Eases channel filter requirements to lower die size \& cost

\section{Principle of Code Phase Shift Keying}

The starting position of a single pn sequence is modulated with the transmitted data

- Multiple bits may be sent in a single symbol time $\rightarrow$ better battery life

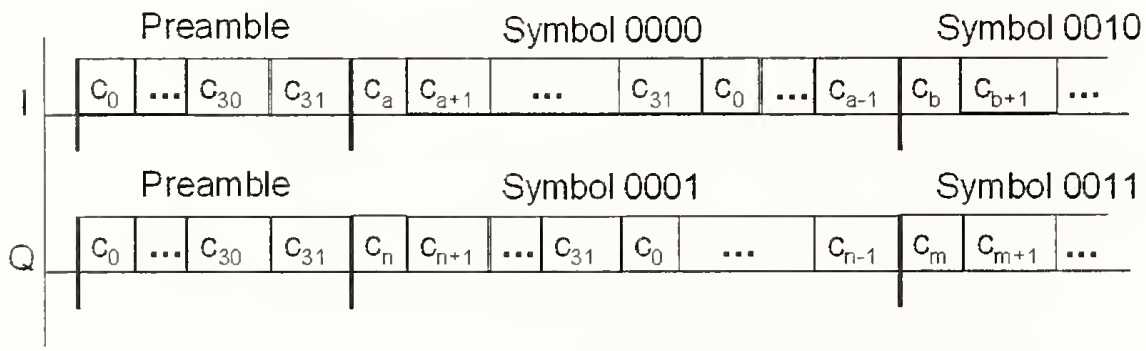


- The IEEE 802.15.4 web site: http://www.ieee802.org/15/pub/TG4.html 


\subsection{Wireless Implementations for an Open Standard Sensor Bus}

\section{Wireless Implementations of an Open Standard Sensor Bus}

Wireless 1451 Workshop

Sensors Expo

October 4, 2001

Michael R. Moore

Oak Ridge National Laboratory'

\section{Wireless Sensor Networks Are a Key Element of Industries of the Future}

- What They Will Provide

- Reduced Install Cost

- Greater Flexibility

- What It II ill Take

- Short Term

"Higher Reliability

\Small, Fast and Cheap (Pick any two)

- Long Term

》Improved Inter-System Compatibility (EMC/TT)

) Standardization 


\section{Who Needs Sensor Networks?}

- Industry

- Science

- Military

Standards Are Necessary to Reach Long-Term Goals

- Provide Commonly Accepted Infrastructure

- Can Erolve With Technology

So we will examine a couple of standards

families --

IEEE 802 and IEEE 1451 


\section{The IEEE 802 Family Has Successfully Evolved With Technology}

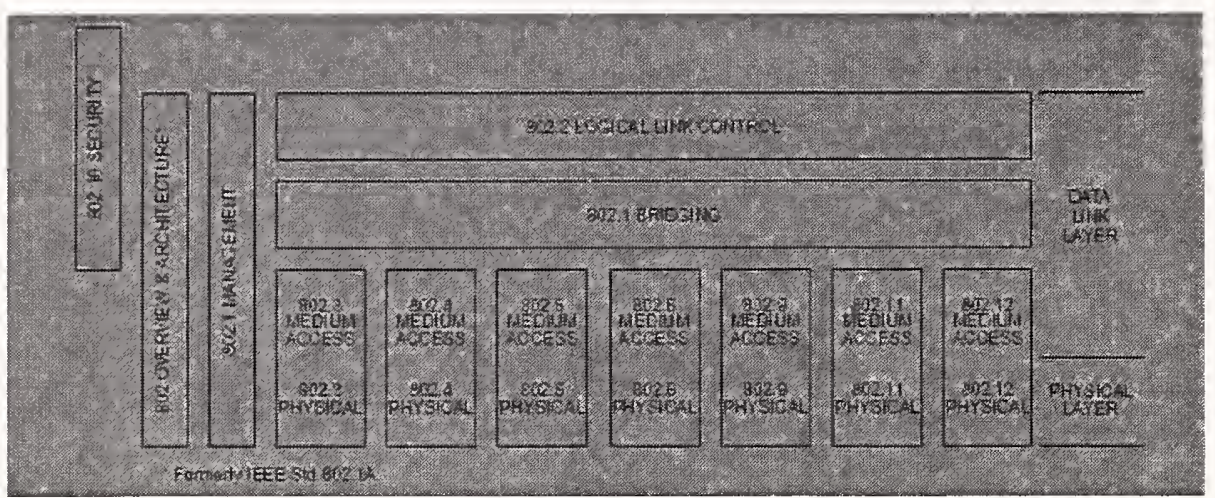

\section{Candidate $1451 \mathrm{MAC} / \mathrm{PHY}$}

From Other Wireless Standards

(Technology Issues)

\begin{tabular}{||l|c|c|c|c|c|c||}
\hline Std & OFDN & FHSS & DSSS & GHz & Size & Mbps \\
\hline IS-95 & & & $x$ & $1+-$ & Cell & $0 . x$ \\
\hline Bluetooth & & $x$ & & 2.45 & PAN & 1 \\
\hline P802.15 & & $x$ & & 2.45 & PAN & 1 \\
\hline P802.16b & $\mathrm{x}$ & & & 5 & WAN & 54 \\
\hline $802.11 \mathrm{a}$ & $\mathrm{x}$ & & & 5 & LAN & 54 \\
\hline 802.11 & & $\mathrm{x}$ & $\mathrm{x}$ & 2.45 & LAN & 1.2 \\
\hline $802.11 \mathrm{~b}$ & & & $\mathrm{x}$ & 2.45 & LAN & 5.5 .11 \\
\hline
\end{tabular}

OFDM - Orthogonal Frequency Division Multiplexing

FHSS - Fr equency Hopping Spread Spectrom

DSSS - Dir ect Sequence Spr ead Specti um 


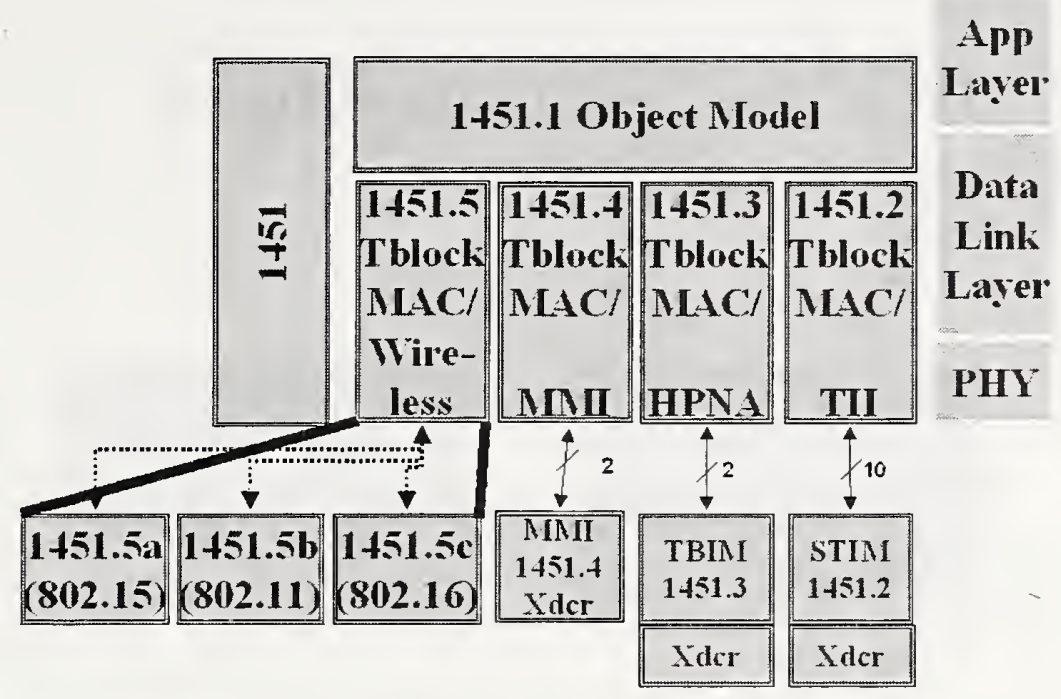

\section{Size of Network vs PHY}

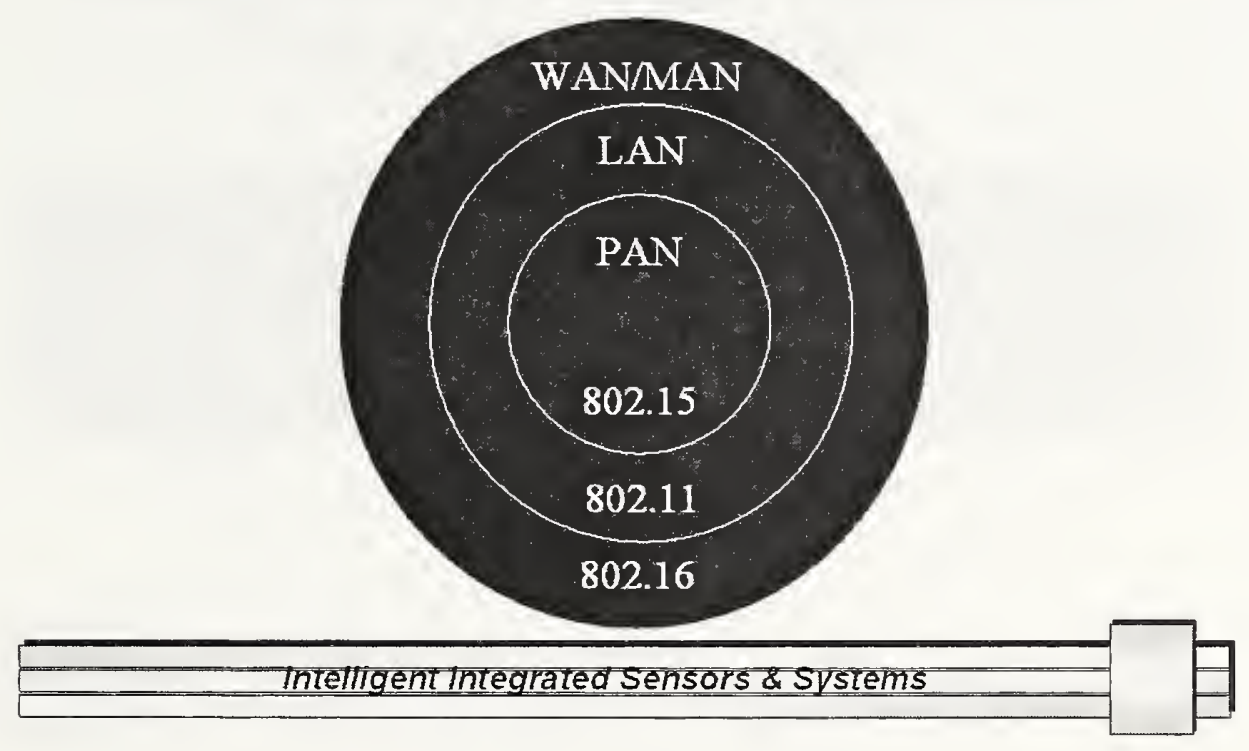




\section{IEEE 802 Wireless Projects}

- IEEE S02.15 (Bhuetooth)

- IEEE 802.11

"Clause 14-1.2 Mbps FHSS LAN MAC for $2.4 \mathrm{GHz}$

n Clause 15-1,2 Mbps DSSS LAN for $2.4 \mathrm{GHz}$

- IEEE 802.11a

"Adds Clause $17-54 \mathrm{Mbps}$ OFDM LAN for $5 \mathrm{GHz}$

- IEEE \$02.11b

"Adds Clause $18-5.5$ and $11 \mathrm{Mbps}$ DSSS for $2.4 \mathrm{GHz}$

- IEEE 802.16 (task group 4) Wireless High-Speed Unlicensed

Metropolitan Area Network (Wireless HUM.AN)

" MAC: IEEE 802.16

"PHY: IEEE 802.11a, ETSI BRAN HIFERLAN/2

Finding the Right RF Technology for the Application

- Conventional Narrorrband

- Frequency-Hopping Spread Spectrum

- Direct-Sequence Spread Spectrum

- Orthogonal Frequency Division Multiplexing 


\section{DSSS vs FHSS}
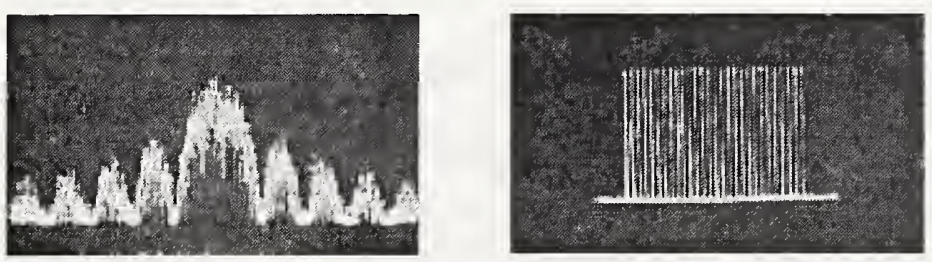

\section{Picking the Right Technology is Very Application Dependent}

\begin{tabular}{|c|c|c|c|c|c|}
\hline $\begin{array}{c}\text { Typical } \\
\text { Rank }\end{array}$ & $\begin{array}{c}\text { Spectral Eff. } \\
\text { (Bits/sec/Hz) }\end{array}$ & $\begin{array}{c}\text { Non- } \\
\text { interfering }\end{array}$ & $\begin{array}{c}\text { Power } \\
\text { Req'd }\end{array}$ & $\begin{array}{c}\text { Data } \\
\text { Reliability }\end{array}$ & $\begin{array}{c}\text { Effective } \\
\text { Range }\end{array}$ \\
\hline Best & OFDM & DSSS & FHSS & OFDM & OFDM \\
\hline Median & DSSS & FHSS & DSSS & DSSS & DSSS \\
\hline Worst & FHSS & OFDM & OFDM & FHSS & FHSS \\
\hline
\end{tabular}




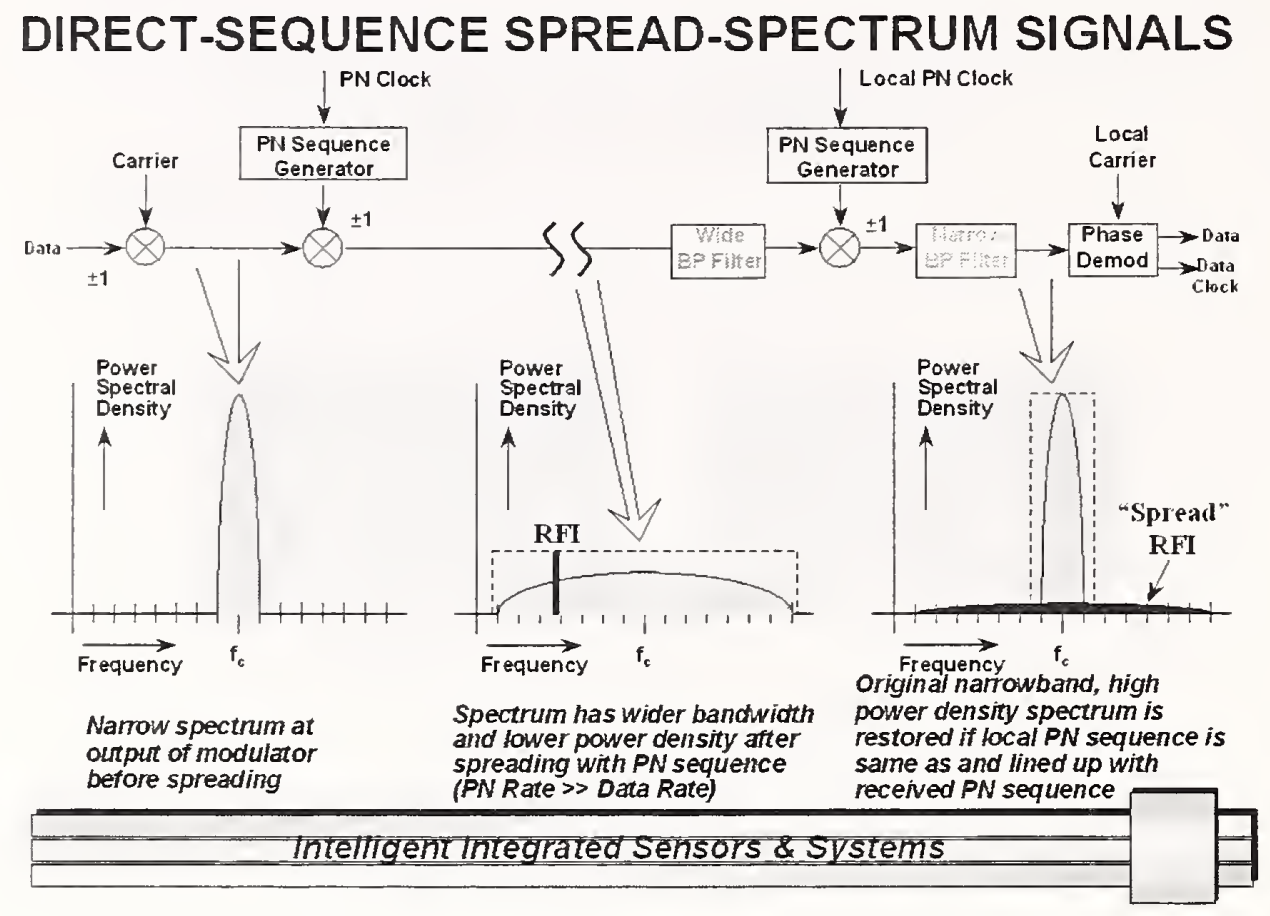

\section{Conclusions}

- Wireless Sensor Networks Are a Key Part of Future Industries (and for the Military)

- Short-Term Goals Focus on Reliable Links and Reasonable Costs of Individual Systems so that Industries Will Accept Them.

Long-Term Goals (Inter-System Compatibility) Require Standards Efforts and Improred RF Technologies. 


\title{
4.7 Review Issues \& Discussions from First Workshop
}

\section{Review Issues \& Discussions from First Workshop}

\author{
James D. Gilsinn \\ National Institute of Standards \& Technology \\ Intelligent Systems Division \\ james.gillsinn@nist.gov
}

\section{Overview}

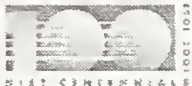

- The first Wireless Sensing Workshop was held on June 4, 2001, at the Sensors Expo Conference at the Rosemont Convention Center in Chicago. IL

- The workshop was organized in order to explore industry's interest in wireless sensing.

- 90 people attended the workshop representing:

- Manufacturing, Process Control. Aerospace. Home Automation. Automotive, and Government 


\section{Overview (cont'd)}

- Overview of IEEE 1451

- Application of IEEE 1451.1

- Review of Current Technologies

- Wireless Ethernet (IEEE 802.11x)

- Bluetooth

- Hardware \& Software tools

- Proposed P1451.5 wireless standard

- Open forum \& discussion

\section{Proceedings}

- Paper Copy of Proceedings

- Contact Kang Lee, kang.lee ânist.gov

- CD Copy of Proceedings

- Available here, or

- Contact James Gilsinn, james gilsinnànist.gov

- Web Accessible Copy of Proceedings

- Available online at http. iee 1451.nist.gor 


\section{Why Use Wireless?}

- Some attendees questioned whether wireless communications should be used for sensors

- Wireless may not be the best solution for all cases

- Many cases may allow for less deterministic communications. These may present possibilities where wireless is a best-fit solution

- Large facilities

- Low-speed, long-range communications

- Hazardous environments

\section{Data Reliability}

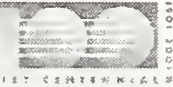

- Data reliability was the largest issue raised

- Data reliability depends on:

- Availability of the wireless signal (hardware)

- Integrity of the data message (software)

- Confidentiality of the data message (software)

- Availability

- Many sensors use ISM band

- ISM band is free. but full of other users

- Some standards allow for signal degradation 


\section{Data Reliability (cont'd)}

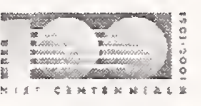

- Data Integrity and Confidentiality

- Error checking of data to prevent bad data packets

- Algorithms arailable to correct errors in the data

- IEEE 1451.2 specification for Transducer Electronic Data Sheet (TEDS) has simple error checking

- Wireless communication lends itself to confidentiality problems

- Vendors should incorporate additional security features to prerent industrial espionage

\section{Bandwidth Requirements}

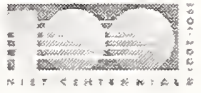

- An informal survey of the bandwidth requirements for workshop attendees was conducted

- Bandwidth

Interested Parties

$-<=300 \mathrm{bps}$

$63^{\circ} \%$

-300 bps $-50 \mathrm{kbps}$

$25^{\circ} \%$

- 50 kbps - 250 kbps

$3^{\circ} \%$

- $250 \mathrm{kbps}-1.5 \mathrm{M}$.

$6 \%$

$-\therefore 1.5 \mathrm{Mlbps}$

$3^{\circ} \%$ 


\section{Where Should Wireless Be Located?}

- The workshop attendees had varying opinions on where wireless communications should be incorporated into a wireless sens or system

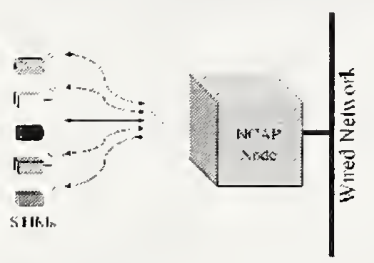

Wueless STMS

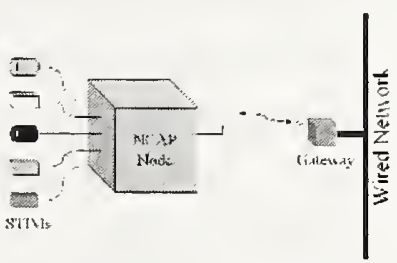

Wireless NCAP Node

\section{Devices Per Node}

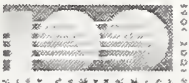

- NCAP nodes allow multiple sensors to be attached to the network using a common point of access

- An informal survey was taken to determine how many sensors per NCAP node users wanted

- Sensors Node

Interested Parties

$-8$ $26^{\circ}{ }_{0}$

$-32$ $53^{\circ} 0$

$-256$ $21 \%$ 


\section{Transmission Power vs. Battery Lifetime \& Safety}

- Some wireless communication standards use $100 \mathrm{~mW}$ of transmission power

- Some sensors expected to run years before replacing batteries

- Batteries lifetime may be reduced because of transmission power

- $100 \mathrm{~mW}$ transmission power may be too high in hazardous environments

\section{"Hot" Wireless Technology}

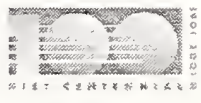

- Although Bluetooth and 802.11 may not be exactly what sensor vendors want they may help bring wireless communications to sensors

- Sensor manufacturers and vendors need to consider development cost vs. performance benefits of using standardized technology rs. ASIC chips

- Derelopment systems for some standardized technology may be prohibitively expensive 


\section{ISM Band Users}

- The Industrial, Scientific, and Medical (ISM)

bands setup by the FCC are unlicensed

- Many consumer \& commercial devices are designed to use these frequencies

- $900 \mathrm{MHz} \quad$ Cell phones, portable phones, home electronics, spread spectrum

- $2.4 \mathrm{GHz} \quad$ Portable phones, spread spectrum

$-5 \mathrm{GHz} \quad$ Satellite communications

\section{Are Multiple IEEE 1451 Wireless Standards Needed?}

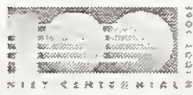

- With the seemingly disjoint worlds of low-speed and high-speed wireless communications. is it possible to create one standard that fits the needs of everyone?

- Will multiple versions of an IEEE 1451 wireless standard be needed to account for the varying needs of its users? 


\section{Other Issues}

- Sensor reconfiguring \& reprogramming

- 2-rray links necessary

- Uplink and downlink can run at different speeds

- Broadcast vs. targeted communications

- High-speed data synchronization by multiple sensors

- Can the NCAP and STIM be combined?

\section{Proceedings}

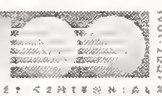

- Paper Copy of Proceedings

- Contact Kang Lee, kang.lee $t$ nist.gov

- CD Copy of Proceedings

- Available here, or

- Contact James Gilsinn, james.gilsinnànist.gor

- Web Accessible Copy of Proceedings

- Available online at http: ieeel 451 .nist.gor 


\section{Workshop Agenda}

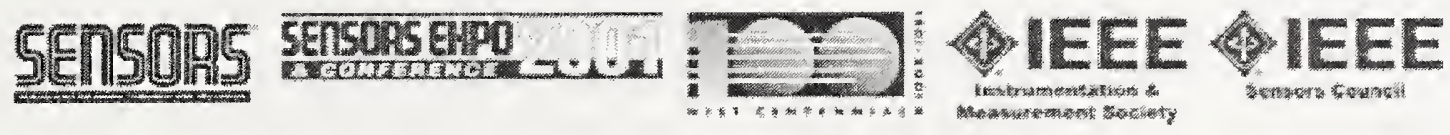

\section{Seasors Expo \& Conference \\ Philadelphia, PA \\ October 4,2001}

\begin{tabular}{|c|c|c|c|}
\hline \multicolumn{2}{|c|}{$\begin{array}{c}\text { Enhancenent of IEEE } 1451.2 \text { A Smart } \\
\text { Transducer Intertace Standard }\end{array}$} & \multicolumn{2}{|c|}{ Second Warkshop on Wireless Sensing } \\
\hline $8000 a m$ & $\begin{array}{l}\text { Introdiction and Purpose } \\
\text { Kang Leg MIST (Moderator) }\end{array}$ & 10: $40 x$ an: & 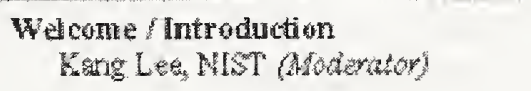 \\
\hline tosan & $\begin{array}{l}\text { Definition of Snart, Review Valuable } \\
\text { Featur of } 451.2 \\
\text { Stun woods }\end{array}$ & $10: 45 x$ & $\begin{array}{l}\text { Synopsis on IEEE 14SI } \\
\text { RId \&chnowath WIST }\end{array}$ \\
\hline $8: 20 \mathrm{axm}$ & $\begin{array}{l}\text { Reguested Chances to } 1451.2 \\
\text { Robat Johnwh, Tulemonitor, Ino. }\end{array}$ & 11.05 and & $\begin{array}{l}\text { Synopsis on Bluet outh } \\
\text { Thurston Brooks, } 3 \text { Techrologies } \\
\text { Intemational }\end{array}$ \\
\hline 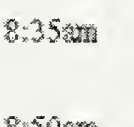 & 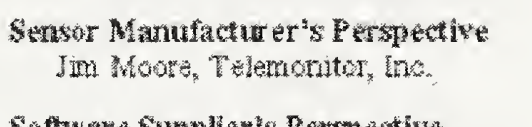 & 11.258 & 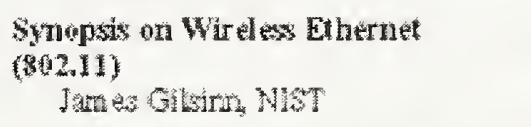 \\
\hline 850 and & $\begin{array}{l}\text { Software Suppliet }{ }^{\text {s }} \text { Perspective } \\
\text { David Pother, National Instrumerts }\end{array}$ & 11.45 ans & $\begin{array}{l}\text { Reliable Wirtess Connectivity for } \\
\text { Sensing \& Control Applications }\end{array}$ \\
\hline grosan & $\begin{array}{l}\text { Procedure for Keview/Renewal or } \\
\text { IEEE, Standards } \\
\text { Stan Woode Agilent Technologies }\end{array}$ & & 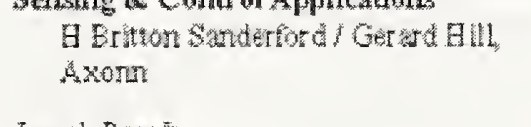 \\
\hline $9: 10$ an & Proposed Euhancements of 1451.2 & 12 100pn & 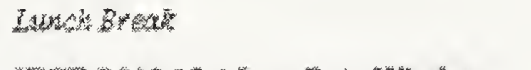 \\
\hline $9030 \mathrm{aras}$ & 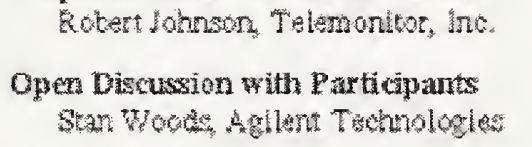 & 1:10pm & $\begin{array}{l}\text { IEEE P802.15.4 Low Rate Wireless } \\
\text { Persinal Area Networks } \\
\text { Karg L Le, NIST }\end{array}$ \\
\hline $10: 20 \mathrm{an}$ & $\begin{array}{l}\text { Wrap-up, Sumarary, and Action } \\
\text { thens } \\
\text { Rong Lee, NIST }\end{array}$ & $1,30 \mathrm{pm}$ & 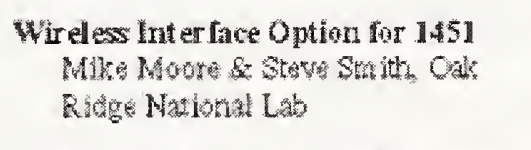 \\
\hline $10 \cdot 30 \mathrm{~mm}$ & Meeting Adjourn & 1.50prat & $\begin{array}{l}\text { Review Issues and Diseussions from } \\
\text { First Workshop } \\
\text { James Gilbirn, Nuso }\end{array}$ \\
\hline & & 2000pri & $\begin{array}{l}\text { Open Group Discussion on New } \\
\text { Istues, New Techulogies, and } \\
\text { Standiardization Approach }\end{array}$ \\
\hline & & $300 \mathrm{per}$ & $\begin{array}{l}\text { IEEE Prowilure and Process for } \\
\text { Setting up Standards Working Group } \\
\text { Kars Le, , NIST }\end{array}$ \\
\hline & & 30 pra & Meeting Adjourn \\
\hline
\end{tabular}




\section{List of Attendees}

\section{Mary Austin}

Pratt \& Whitney

austinma@pweh.com

\section{Ken Ball}

Thurston Brooks

$3 \mathrm{eTI}$

tbrooks@3eti.com

\section{John Cermmzro \\ EC Systems \\ 11601 Interchange Drive \\ Louisville, KY 40229-5791 \\ jcermmzro@InspectionLogic.com}

\section{Steven Chen}

$3 \mathrm{eTI}$

schen@3eti.com

\section{Robert Correa}

Texas Instruments

34 Forest Street, MS 11-07

P.O. Box 2964

Attleboro, MA 02703

rcorrea@ti.com

508-236-2322

\section{John Devitt}

GE

Building ESB, Room 604

1 Research Circle

Niskayuna, NY 12309

devitt@crd.ge.com

518-387-6327

\section{Lee Eccle}

\section{Kevin Finkbiner}

ACT

2001 Fulling Mill Rd.

Middletown, PA 17057

kfinkbiner@actpower.com

717-939-2300

\section{James Gilsinn \\ NIST}

100 Bureau Drive, Stop 8230

Gaithersburg, MD 20899-8230

james.gilsinn@nist.gov

301-975-3865

\section{Tian He}

Kulicke \& Soffa Industries, Inc.

the@kns.com

215-784-6808

\section{Gerard Hill}

Axonn

2021 Lakeshore Drive

New Orleans, LA 70122

gerhill@axonn.com

504-282-8119

\section{Hui Huang}

NIST

100 Bureau Drive, Stop 8230

Gaithersburg, MD 20899-8230

hui-min.huang@nist.gov

301-975-3427

\section{Doug Keene}

Neuron Therapeutics

81 Great Valley Parkway

Malvern, PA 19355

douglaskeene@yahoo.com

215-699-1464

\section{Kang Lee}

NIST

100 Bureau Drive, Stop 8220

Gaithersburg, MD 20899-8220

kang.lee@nist.gov

301-975-6604 


\section{Bill Moore}

431 Indian Creek Rd.

Harleysville, PA 1943?

wbm@walnut-hill.com

\section{Lloyd Powell}

EME Corp.

1197 Baltimore Annapolis Blvd.

Arnold, MD 21012

lpowell@emecorp.com

410-544-8563

\section{Britton Sanderford}

Axonn

2021 Lakeshore Drive

New Orleans, LA 70122

\section{David Stephan}

CME

$290444^{\text {th }}$ Avenue N.

St. Petersburg, FL 33714-6888

dstephan@custom-mfg-eng.com

727-547-9799 

